
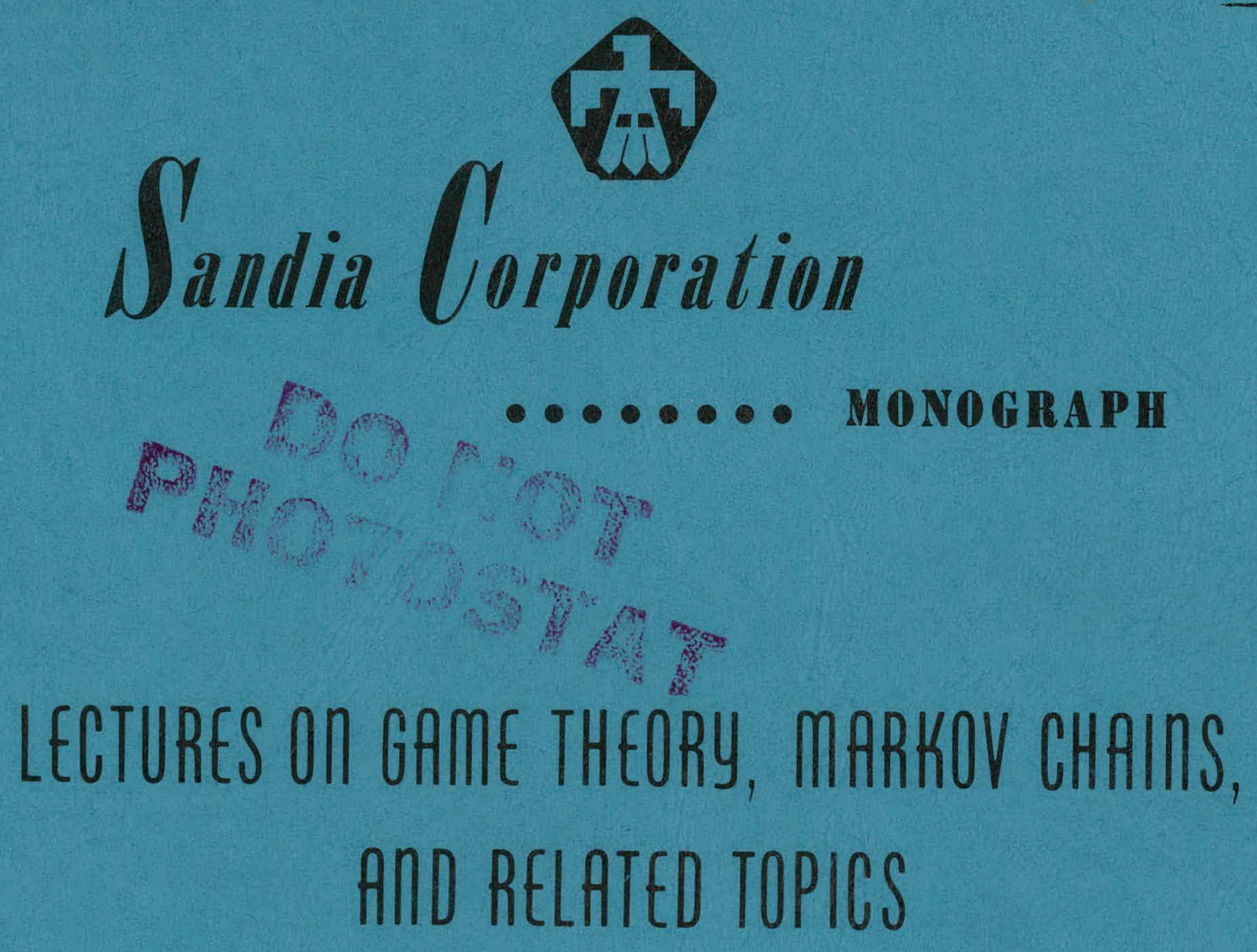

\author{
by \\ Gerald L. Thompson \\ Dartmouth College
}

March 1958 


\section{DISCLAIMER}

This report was prepared as an account of work sponsored by an agency of the United States Government. Neither the United States Government nor any agency Thereof, nor any of their employees, makes any warranty, express or implied, or assumes any legal liability or responsibility for the accuracy, completeness, or usefulness of any information, apparatus, product, or process disclosed, or represents that its use would not infringe privately owned rights. Reference herein to any specific commercial product, process, or service by trade name, trademark, manufacturer, or otherwise does not necessarily constitute or imply its endorsement, recommendation, or favoring by the United States Government or any agency thereof. The views and opinions of authors expressed herein do not necessarily state or reflect those of the United States Government or any agency thereof. 


\section{DISCLAIMER}

Portions of this document may be illegible in electronic image products. Images are produced from the best available original document. 
S A N D I A C OR P O R A T I O N M O N O G R A P H

¿ECTURES ON GAME THEORY, MARKOV CHAINS, AND RELATED TOPICS

BY

\author{
GERALD L. THOMPSON \\ Assistant Professor in Mathematics \\ Dartmouth College \\ and \\ Sandia Conporation Consultant
}

June 1958

Work Performed Under AEC Contract AT-(29-1)-789 
THIS PAGE

\section{WAS INTENTIONALLY LEFT BLANK}


TABLE OF CONTENTS

PART ONE: ON METHODS OF OBTAINING A RANKING FROM A PAIRWISE REDUNDANT ORDERING OF MEMBERS OF A SET

INTRODUCTION . . . . . . . . . . . . . . . . . . . . . . . . . 7

CH I. THE REGULAR CASE . . . . . . . . . . . . . . . . . . 8

A. Definitions and Notation . . . . . . . . . . . . . . 8

B. The Wei-Kendall Ranking Method . . . . . . . . . . 10

C. The Dominance Ranking Method . . . . . . . . . . . 12

CH II. THE GENERAL CASE FOR PAIRWISE REDUNDANT RELATIONS . . . . . . . . . . . . . . . . . . . . . . 17

A. More on Order Relations. . . . . . . . . . . . . . . 17

B. Equivalence Classes and Their Partial Ordering . . . . 17

CH III. EXTENSIONS, EXAMPLES, AND COMPARISONS . . . . $21^{\prime}$

A. Multiple Judgments ... . .. . . . . . . . . . . . 21

B. Examples. . . . . . . . . . . . . . . . . . . . . . 21

1. Comparison of the Scoring Methods of CH I . . . . 21

2. Multiple Judging . . . . . . . . . . . . . . . . 22

3. Ties. . . . . . . . . . . . . . . . . . . . . . 23

C. Comparisons and Extensions . . . . . . . . . . . . . 24

REFERENCES FOR PART ONE . . . . . . . . . . . . . . . 26

PART TWO: NOTES ON FINITE MARKOV CHAINS

CH IV. REGULAR MARKOV CHAINS . . . . . . . . . . . . . . . 29

$\mathrm{CH}$ V. NONREGULAR CHAINS . . . . . . . . . . . . . . . . 31

A. The Classification of States . . . . . . . . . . 31

B. Definitions . . . . . . . . . . . . . . . . . . . . 31

C. Absorbing Markov Chains . . . . . . . . . . . . . . 32

CH VI. THE OPEN LEONTIEF MODEL . . . . . . . . . . . . . 36

CH VII. SEMIMARTINGALES OF MARKOV CHAINS . . . . . . . . 39

REFERENCES FOR PART TWO . . . . . . . . . . . . . . . . . . 44 
PART THREE: NOTES ON.GAME THEORY

Page

CH VIII. MATRIX GAMES . . . . . . . . . . . . . . . 45

A. Introduction . . . . . . . . . . . . . . . . . . 45

B. Proofs of the Main Theorem . . . . . . . . . . . . 46

Stiemke's Theorem . . . . . . . . . . . . . . . 47

C. Other Theorems . . . . . . . . . . . . . . . 47

1. The Strong Minmax Theorem . . . . . . . . 47

2. Dimension Theorems . . . . . . . . . . . . 48

3. Kernels... . . . . . . . . . . . . . . . . 48

4. Linear Programming . .. . . . . . . . . . . 49

D. Computational Methods . . . . . . . . . . 50

CH IX. THE EFFECT OF PSYCHOIMRIRAA ATTITUDES ON TIIE OUTCOME OH CIAMES . . . . . . . . . . . . . . . . 53

A. Characterization of Strategy-Preserving Utility

Functions . . . . . . . . . . . . . . 53

B. Psychological Attitudes and Utility Functions . . . . 56

C. Examples . . . . . . . . . . . . . . . 58

1. The Lottery Game . . . . . . . . . . . . 58

2. The Insurance Game . . . . . . . . . . . . . 59

3. Colonel Blotto . . . . . . . . . . . . . . . . 60

D. A Sequence Game . . . . . . . . . . . . . . . 61

$\mathrm{CH}$ X. GAMES IN EXTENSIVE FORM . . . . . . . . . . . . . 63

CH XI. MATHEMATIÇL ELUNUMTCS AND MATRIY GAME

THEORY. . . . . . . . . . . . . . . . . . . . . 70

A. Introduction . . . . . . . . . . . . . . . . . 70

B. A Generalization of the Von Neumann Model of an Expanding Economy . . . . . . . . . . . . . 70

C. The Walras-Wald E'conomic Model. . . . . . . . 78;

REFERENCES FOR PART THREE . . . . . . . . . . . . . . . . . . 83 


\title{
LECTURES ON GAME THEORY, MARKOV CHAINS, AND RELATED TOPICS
}

\author{
by \\ GERALD L. THOMPSON \\ Assistant Professor in Mathematics \\ Dartmouth College \\ and \\ Sandia Corporation Consultant
}

\begin{abstract}
A BSTRACT
This memorandum contains notes on nine lectures delivered at Sandia Corporation in August 1957. As indicated in the table of contents, three main subjects were discussed. Part One contains the manuscript of a paper concerning a judging problem. Part Two is concerned with finite Markov-chain theory and discusses regular Markov chains, absorbing Markov chains, the classification of states, application to the Leontief input-output model, and semimartingales., Part Three contains notes on game theory and covers matrix games, the effect of psychological attitudes on the outcomes of games, extensive games, and malrix game theory applied to mathematical economics. These notes vary in completeness, but in most cases references to the literature are given.
\end{abstract}




\section{THIS PAGE WAS INTENTIONALLY LEFT BLANK}


PART ONE

\section{ON METHODS OF OBTAINING A RANKING FROM A PAIRWISE REDUNDANT ORDERING OF MEMBERS OF A SET}

\section{INTRODUCTION}

Consider a finite set of objects and an irreflexive, binary ordering relation defined for some pairs of elements in the set. For example, the set could consist of chickens, chess players, production processes, football teams, etc., and the corresponding ordering relations would be pecks, defeats, is superior to, beats, etc. Such situations have recently been of interest to statisticians (see References 1-7) and sociologists (see References 8 and 9). (See also Reference 10.) The question to he answered in such cases is that of determining, when possible, a rank ordering or ranking of the objects in the set using only the more or less incomplete information given by the ordering relation. One important special case is paired comparisons in which the ordering is defined between every pair of objects in the set.

This paper presents mathematical treatment of the Wei-Kendall method (Reference 3 ) of obtaining the ranking and gives it two different characterizations. This part also presents a new ranking method and shows that it contains the Wei-Kendall procedure as a special case. Computational procedures, involving the solution of certain matrix games, are given for these methods.

Although the methods presented here are not directly related to those previously considered by some statisticians for analyzing paired-comparison data, such as the MostellerThurstone method (Reference 6) or the rankit method (Reference 2), the results seem to be nearly the same (see Chapter III, Section 3).

Part of the material presented here is based on the elementary but important mathematics of order relations that has recently been included in some experimental mathematical writing for sophomores (Reference 11). In fact, an early version of this material appears there. This writing was prepared under the sponsorship of the Committee on the Undergraduate Program of the Mathematical Association of America. Readers who find this material of interest may also wish to support the introduction of such experimental material into the undergraduate currïculum. 
CHAPTER I

THE REGULAR CASE

\section{A. Definitions and Notation}

Consider a set $X$ and a binary ordering relation $R$. The relation is reflexive if $x R x$ for all $x$ in $X$; it is irreflexive if $x R x$ is false for all $x$ in $X$. The relation is symmetric if $x R y$ implies $y R x$; it is asymmetric if $x R y$ implies "not $y R x$. " The relation is transitive if $x R y$ and $y R z$ imply $x R z$. It is pairwise complete if, for $x \neq y$, exactly one of the relations $x R y$ or $y R x$ holds. It is pairwise redundant if, for $x \neq y$, at least one of the relations $x R y$ or $y R x$ holds. Thus a pairwise complete relation is also pairwise redundant.

Consider a set $A$ of $n$ objects $A_{1}, A_{2}, \ldots, A_{n}$, and an irreflexive binary ordering relation $\gg$, called a dominance relation. The effect of the dominance relation can be completely summarized by means of an $\overline{n \times n}$ dominance matrix $D$ with entries $d_{i j}$ where $d_{i j}=1$ if $A_{i} \gg A_{j}$ and is zero otherwise. Because the relation is irreflexive, we have

\section{(a) (Irreflexive) For all $i, d_{i j}=0$.}

If the relation is pairwise complete or pairwise redundant, we will have

$$
\begin{aligned}
& \text { (b) (Pairwise complete) For } i \neq j, d_{i j}=1 \text {, if and only if } d_{j i}=0 \text {. } \\
& \text { (c) (Pairwise redundant) For } i \neq j \text {, if } d_{i j}=0 \text {, then } d_{j i}=1 .
\end{aligned}
$$

Note that the nonzero entries in the ith row of $D$ indicate the members that $A_{i}$ dominates and the nonzero entries in the $j$ th column of $D$ indicate the members that dominate $A_{j}$.

In Chapter I, the only assumption consistently made about the dominance relation is that it is irreflexive. A special assumption will be made about the matrix $D$, namely that it is regular (see below), but this assumption will be dropped in Chapter 11 . Note that it is not required that the relation be transitive, and most interest lies in cases in which it is not.

Say that $A_{i}$ has a k-stage dominance over $A_{j} \quad$ (see Reference 10, page 309) if there is a chain of relations

$$
A_{i}=A_{i_{1}} \gg A_{i_{2}} \gg \ldots \gg A_{i_{k}+1}=A_{j}
$$


with elements $A_{i p}$ chosen from the set $A$. If the elements in the chain are all distinct, call the $\mathrm{k}$-stage dominance nonredundant; otherwise call it redundant. It is easy to show (see Reference 10 , page 309 ) that the $i, j$ th entry of $D^{k}$ gives the total number of (redundant and nonredundant) $k$-stage dominances that $A_{i}$ has over $A_{j}$. Methods of counting the number of nonredundant k-stage dominances have been developed by Ross and Harary in Reference 9 .

In the sequel, a theorem (essentially due to Frobenius) is needed about nonnegative matrices that are regular.

DEFINITION. - A matrix $A$ is said to be regular if $A^{p}>0$ for some positive integer p.

THEOREM 1. -- If $A$ is a nonnegative regular matrix then

(a) It has a unique positive characteristic value $k$ of largest absolute value (the principal characteristic value).

(b) To $\mathrm{k}$ can be associated a row characteristic vector $\mathrm{x}>0$ and a column characteristic vector $\mathrm{y}>0$.

(c) For certain such characteristic vectors

$$
\lim _{n \rightarrow \infty} \frac{1}{k^{n}} A^{n}=y x .
$$

We outline a proof of this theorem based on References 12 and 13. Theorem I of Reference 12 and its proof carry over verbatim if the word "indecomposable" is replaced by the word "regular." If $\mathrm{k}$ is not the unique characteristic vector of largest absolute value, then there are $m(\geq 2)$ such characteristic values of equal absolute value. By Theorem II in Reference 12 (prover in Reference 13, p 646-7), there is a permutation $\Pi$ so that

$$
\Pi A \Pi^{-1}=\left(\begin{array}{ccccc}
0 & A_{12} & 0 & \cdot & 0 \\
0 & 0 & A_{23} & \cdot & 0 \\
\cdot & \cdot & \cdot & \cdot \\
0 & 0 & 0 & \cdot & A_{m-1, m} \\
A_{m 1} & 0 & 0 & \cdot & 0
\end{array}\right)=B .
$$

But then $A=\Pi^{-1} B \Pi$ is clearly not regular since $A^{p}=\Pi^{-1} B^{p} \Pi$ and $\Pi$ is a permutation matrix. This outlines a proof of (a) and (b). 
To prove (c), write $A$ in Jordan canonical form as

$$
\mathrm{TA} \mathrm{T}^{-1}=\left(\begin{array}{cccccc}
\mathrm{k} & 0 & \cdot & \cdot & \cdot & 0 \\
0 & \mathrm{~J}_{2} & \cdot & \cdot & \cdot & 0 \\
\cdot & \cdot & \cdot & \cdot & \cdot & \cdot \\
0 & 0 & \cdot & \mathrm{J}_{\mathrm{i}} & \cdot & 0 \\
\cdot & \cdot & \cdot & \cdot & \cdot & \cdot \\
0 & 0 & \cdot & \cdot & \cdot & \mathrm{J}_{\mathrm{q}}
\end{array}\right)=\mathrm{C},
$$

where $T$ is a suitable nonsingular complex matrix. Also

$$
J_{i}=\left(\begin{array}{ccccccccc}
a_{i} & 1 & 0 & \cdot & \cdot & \cdot & 0 & 0 \\
0 . & a_{i} & 1 & \cdot & \cdot & \cdot & 0 & 0 \\
\cdot & \cdot & & \cdot & \cdot & \cdot & \cdot & \cdot \\
0 & 0 & 0 & \cdot & \cdot & \cdot & a_{i} & 1
\end{array}\right)
$$

where the $a_{i}$ 's are characteristic roots of. A with $\left|a_{i}\right|<k$. Since $A^{-1}=T^{-1} C$, the first column of $T^{-1}$ is a characteristic vector associated with $k$. And since $T A=C T$, the first row of $T$ is a characteristic vector associated with $k$. Because $A^{p}=T^{-1} C^{p} T$ is positive for $p$ large, these characteristlc vectors are pusilive, call them $x$ (thc firat row of $T$ ) and $y$ (the first column of $I^{-1}$ ). Now from the above equaliuis and the fact that $\left|a_{i}\right|<k$, we have $\lim \frac{1}{k^{n}} A^{n}=\lim \frac{1}{k^{n}} \cdot I^{\prime-1} C^{H^{\prime}} I^{\prime}=y x$, completing the prouf.

\section{B. The Wei-Kendall Ranking Method}

The usual method of obtaining a ranking from an ordering of a set of objects is to give a method of assigning a numerical score to each of the objects and then ranking them according to the score. Wei and Kendall have proposed (see Reference 3) the following method for as signing scores. A's an initial score, assign to each ruember the number of other members of the set over whom he has a one-stage dominance, plus a score of $1 / 2$ for dominating himself. The assignment of $1 / 2$ for dominating himself is, of course, arbitrary and it will be seen later that it does not matter what this number is as long as it is positive. It can be seen that the initial score vector is given by

$$
s_{1}=\left(D+\frac{1}{2} I\right) e
$$


where $e$ is the $n$-component column vector each of whose components is equal to one. Then as a second score, assign to each member one-half his own initial score plus the initial scores of each of the people he dominates in one stage; the second-score vector is given by

$$
s_{2}=\left(D+\frac{1}{2} I\right)^{2} e .
$$

Now keep iterating this procedure until there is no change in the ordering of the people by the scores so obtained. Thus, after $m$ iterations the score vector is

$$
\mathrm{s}_{\mathrm{m}}=\left(\mathrm{D}+\frac{1}{2} \mathrm{I}\right) \mathrm{m} \mathrm{e}
$$

After the matrix is raised to a sufficiently high power, the order of the components of the scoring vector will remain fixed, at least if the matrix is indecomposable and its principal column characteristic vector has distinct components (Kendall, Reference 3). The next theorem establishes this result by a different method than Kendall used and also provides an independent characterization of the score vector.

THEOREM 2. - Let $\mathrm{D}$ be the matrix of an irreflexive binary ordering relation, assume that $D+\frac{1}{2} I$ is a regular matrix, let $k$ be its principal characteristic root, and let $y$ be a characteristic column vector of $D+\frac{1}{2} I$ associated with $k$. Then if we assign as a score t's the members of $A$ the components of the vector $y$, and if the components of $y$ are distinct, the ordering determined in this manner will agree with the final ordering as determined by Equation 4.

PROOF. - - If $\mathrm{D}+\frac{\mathrm{I}}{2} \mathrm{I}$ is regular, Theorem 1 implies

$$
\lim _{m \rightarrow \infty}\left(\frac{1}{k}\right)^{m} \quad\left(D+\frac{1}{2} I\right)^{m} e=y x e=c y,
$$

where $\mathrm{k}$ and $\mathrm{y}$ are as in the statement of the theorem, $\mathrm{x}$ is the characteristic row vector associated with $k$, and $c$ is a constant equal to xe. Therefore, for $m$ large, the entries of the vector $\left(D+\frac{1}{2} I\right)^{m_{e}}$ must be ordered in the same way as are the entries of the vector $y$. This completes the proof.

If two of the components of $y$ are equal, then the behavior for large $m$ of the corresponding components of $s_{m}$ is not clear. In any case, we assign $y$ as the score vector and the corresponding individuals are tied in the ranking.

The only ad hoc thing about this method of obtaining the ranking is the arbitray choice of giving each member half of his old score plus the scores of the people he dominates. The 
next theorem shows that the number $1 / 2$ might be chosen to be any other positive number without changing the final scoring.

THEOREM 3. -- If each person is given a positive multiple $r$ of his old score plus the scores of the members he dominates on each step of the scoring, the final ranking is the same as that obtained with $r=1 / 2$.

PROOF. - - Under the assumptions of the theorem, the score vector after $m$ iterations will be

$$
s_{m}=(D+r I)^{m} e .
$$

Since the entries of $D+r I$ are numegalive il is obvious that $D+r I$ will be regular if and only if $D+\frac{1}{2} I$ is regular. Hence, by the same kind of proof as that of Theorem 1 ,

$$
\lim _{m \rightarrow \infty}\left(\frac{1}{k}\right)^{m}(D+r I)^{m}=y x e=c y,
$$

where $\mathbf{k}$ is the principal characteristic value of $\mathbf{D}+\mathrm{rI}, \quad \mathbf{x}$ and $\mathbf{y}$ are the associated row and column characteristic vectors, and $c=x e$. However, since $(D+r I) y=k y$ implies $D y=(k-r) y$, we see that $y$ is also a characteristic column vector of $D$ associated with $k-r$. It is easy to see that $k-r$ is the principal characteristic value of $\mathrm{D}$ since otherwise $\mathbf{k}$ would not be the principal characteristic value of $\mathrm{D}+\mathrm{rI}$. This show's that the ordering is independent of the value of $r$ and completes the proof.

COROLLARY 1. - - The Wei-Kendall scoring vector may be obtained by computing a characteristic column vector associated with the principal characteristic value of the matrix D.

This corollary gives a characterization of the Wei-Kendall scoring vector and also a method of computing it. A second characterization is given in the next section.

C. The Dominance Ranking Method

Here a new method is proposed for obtaining a ranking from an ordering of a set. It is a generalization of the methods proposed in Exercises 10-12 on pages 314-15 of Reference 10. Initially the method will depend upon a regularity assumption but the latter will be removed in Chapter 11. Theorem 6 shows that the Wei-Kendall method is a special case of the dominance method.

The dominance method is the following: Pick a positive number $r$, and then consider the infinite sum $D+r D^{2}+r^{2} D^{3}+r^{3} D^{4}+\ldots$. If the value of $r$ was chosen so that this infinite sum converges, then the scoring vector for the members of the set $A$ is defined to be

$$
s:=\left(D+r D^{2}+r^{2} D^{3}+\ldots\right) e
$$


Observe that this method of judging gives as a score to each member the number of members of the set he dominates in one stage, plus $r$ times the number he dominates in two stages, plus $r^{2}$ times the number he dominates in three stages, etc. Conditions for the convergence of the infinite sum and an expression for its sum are given in the next theorem.

THEOREM 4. -- Let $\mathbf{D}$ be the matrix of an irreflexive binary ordering relation. Assume that $D$ is regular, $k$ its principal characteristic value, and let $r$ be any positive number. Then

(a) A necessary and sufficient condition that the infinite sum in Equation 8 converges is that $r<\frac{1}{\mathrm{k}}$.

(b) If the sum converges, then the scoring vector is

$$
\begin{aligned}
s & =\left(D+r D^{2}+r^{2} D^{3}+\ldots\right)_{e} \\
& =D(I-r D)^{-1} e .
\end{aligned}
$$

PROOF. - - The following identity may be established by an induction argument:

$$
\left(D+r D^{2}+\cdots+r^{n-1} D^{n}\right)(I-r D)=D-r^{n} D^{n-1} \text {. }
$$

If $\mathrm{r}<\frac{1}{\mathrm{k}}$, we see that $\frac{1}{\mathrm{r}}$ cannot be a characteristic root of $\mathrm{D}$, hence the matrix $I$ - $r D$ has a nonzero determinant and also has an inverse. If Equation 10 is multiplied by that inverse the result is

$$
D+r D^{2}+\ldots+r^{n-1} D^{n}=D\left(I-r^{n} D^{n}\right)(I-r D)^{-1}
$$

Since $(1 / k)^{n}>r^{n}$, we see from Theorem 1 that the term $r^{n} D^{n}$. tends to zero. Hence, for $n$ sufficiently large, we have $0 \leq I-r^{n} D^{n} \leq I$ which implies that

$$
D+r D^{2}+\ldots+r^{n-1} D^{n} \leq D(I-r D)^{-1}
$$

Therefore the infinite sum converges, since it has all positive terms and is bounded above. If we look again at Equation 11, we see that the infinite sum converges to the value claimed for it in Equation 9, which proves (b) and the sufficiency part of (a). For the necessity part of (a), observe that if $r=1 / k$ then the $n$th term of the infinite sum in Equation 8 tends to a nonzero value, and if $r>1 / k$ it tends (by Theorem 1 ) to infinity. In either case the infinite sum does not exist. This completes the proof of (a).

As was seen in Corollary 1, the Wei-Kendall scoring vector is proportional to the characteristic column vector associated with the principal characteristic root of $D$. The next theorem shows that the dominance scoring vector is proportional to the vector Dy where $y$ is the optimal strategy vector for the column player in the matrix game $G=I-r D$. It also shows that if we only wish the ranking, not the actual scores of the members, we can order them according to the components of the vector $y$. First we need a definition. 
DEFINITION. - - Let $\mathrm{H}$, be an $\mathrm{n} \times \mathrm{n}$ matrix with entries $\mathrm{h}_{\mathrm{ij}}$, and let $\mathrm{H}_{\mathrm{ij}}$ be the cofactor of the element $h_{i j}$. Denote by $H^{*}$ the transposed cofactor matrix $\mathbf{H}^{*}=\left\|\mathrm{H}_{\mathrm{ij}}\right\| \operatorname{tr} . \quad$ (Recall that if $\operatorname{det} \mathrm{H} \neq 0$, then $\mathrm{H}^{-1}=\left(\frac{1}{\operatorname{det} \mathrm{H}}\right) \mathbf{H}^{* *}$.)

THEOREM 5. - - Under the assumptions of Theorem 4, for $r$ sufficiently small (for example, $r<1 /(n-1)$ is always sufficient), the scoring vector Equation 9 is proportional to the vector $\mathrm{Dy}$, where $\mathrm{y}$ is the unique optimal strategy for the column player in the matrix game $G=I-r D$. Moreover, the components of $D y$ and the components of $y$ are ordered in the same way.

PROOF. - - If we use the transposed cofactor matrix, we can write the score vector Equation 9 as $s=\left(\frac{1}{\operatorname{det} G}\right) \mathrm{Dz}$, where $z=G^{*} y$. The vector $z$ has as entries the sums of the cofactors of the columns of the matrix I - rD. These entries will, in turn, be proportional to the components of the optimal strategy for the column players of the matrix. game $G=I-r D$, providing that the matrix itself is a kernel of the game (see McKinsey, Reference 14). We shall show that $r$ can be chosen small enough that the whole matrix is the kernel of the game $G$. We first show that $r$ can be chosen small enough that the value of $G$ is positive. Observe that the game $G$. is not more unfavorable to the row player than the game $\mathrm{C}$ which is an $\mathrm{n} \times \mathrm{n}$ matrix with ones on the main diagonal and all entries off the main diagonal equal to $-r$. It is easy to show (see Reference 10, page 293, Exercise 8) that the value of the game $C$. is equal to $1 / n-[(n-1) / n] r$. Therefore if $r<1 /(n-1)$, the value of $G$ is certainly positive. We next show that for small $r$ every optimal strategy for the row player in the game $G$ puts positive weight on each row of the matrix. Suppose, on the contrary; that he had an optimal strategy that put zero weight on the first row (say). If the column player counters with the (not necessarily optimal) strategy that chooses the first column with probability one, the row player's expectation is at most zero, contradicting the fact that the value of the game $G$ is positive for small enough $r$. Hence for $r$ sufficiently small, the kernel of the game is the whole matrix and optimal strategies for each player are unique.

The column player's optimal strategy $y$ is
\[ y=\frac{1}{K}(I-r D)^{*} e, \]

where

$$
K=\sum_{i, j} G_{i j}
$$

and $G_{i j}=(I-r D)_{i j}$ is the cofactor of the $i, j$ th element of the matrix $G=I-r D$. The quantity $\mathrm{K}$ is nonzero because the whole matrix is a kernel of the game. Thus $s=(\operatorname{det} G) D(I-r D)^{*} e=t D y$, where $t=K(\operatorname{det} G)$ is a constant. Therefore s. is proportional to Dy.

From the identity $(I-r D) y=y-r D y$, we obtain $D y=(1 / r)[y-(I-r D) y]$. Since the kernel of the game is the whole matrix, we have $(I-r D) y=v$; where $v$ is an $n$-component column vector each of whose entries is equal to the value of the game $G$. 
Each component of $y$ is greater than or equal to the value of the game $G=I-r D$ since $y$ is an optimal stategy in that game. Hence $y-v$ is a nonnegative vector. Therefore the members of the set $A$ are ranked in the same way by the vectors $s$ and $y$. (To get the actual scores, one must compute the quantity tDy which is, in general, different from y.) This completes the proof of the theorem.

Theorem 5 gives another computational procedure for finding the ranking, namely, to solve the matrix game I - rD for the column player's optimal strategy. It also shows that the actual score vector obtained by the dominance method may depend upon the value of $r$ chosen.

COROLLARY 2. -- Under the assumptions of Theorem 4, if $r<1 /(n-1)$, then $\mathrm{r}<1 / \mathrm{k}$, where $\mathrm{k}$ is the principal characteristic root of $\mathrm{D}$. Therefore, if $\mathrm{y}$ is the column player's optimal strategy in the game

$$
\mathrm{G}=\mathrm{I}-\frac{1}{\mathrm{n}} \mathrm{D}
$$

then Dy or $y$ gives a ranking of the members of the set A. (Remark: observe that the game Equation 15 can be computed for any problem without finding $k$, a fact that substantially reduces computing time, if only a ranking, not the actual score, is desired.)

PROOF. - - We showed in the proof of Theorem 5 that if $r<1 /(n-1)$, then $\mathrm{G}=\mathrm{I}-\mathrm{rD}$ has positive value and its only kernel is the whole matrix. The value under these conditions is $(\operatorname{det} \mathrm{G}) / \mathrm{K}$ where $\mathrm{K}$ is as in Equation 14. Therefore, $\operatorname{det} G=\operatorname{det}(I-r D) \neq 0$, and $1 / r$ is not a characteristic root for any $r<1 /(n-1)$. Therefore $\mathrm{r}<1 / \mathrm{k}$.

We do not, as yet, have any connection between the Wei-Kendall ranking method and the dominance method. To find a closer connection, reconsider Equation 10. If we multiply it on the right by $\mathrm{G}^{*}=(\mathrm{I}-\mathrm{rD})^{*}$, we obtain

$$
(\operatorname{det} G)\left(D+r D^{2}+\ldots+r^{n-1} D^{n}\right)=D\left(I-r^{n} D^{n}\right)(I-r D)^{*}
$$

This equation holds for any $\mathrm{n}$ and any $\mathrm{r}$. Hold $\mathrm{r}<1 / \mathrm{k}$, and let $\mathrm{n}$ tend to infinity in Equation 16; we obtain

$$
(\operatorname{det} G)\left(D+r D^{2}+\ldots\right)=D(I-r D)^{*}
$$

Although the infinite sum in the second factor on the left-hand side of Equation 17 does not exist if $r=1 / k$ (since, by Theorem 1, its $n$th term does not tend to zero), the righthand side of Equation 17 and hence the left-hand side, tend to limits as $r$ approaches $1 / \mathrm{k}$ from below. (Observe that $\operatorname{det} G$ tends to zero.) We therefore define the dominance score vector for $r=1 / \mathrm{k}$ to be

$$
\mathrm{S}=\mathrm{D}\left(\mathrm{I}-\frac{1}{\mathrm{k}} \mathrm{D}\right)^{*} \mathrm{e}=\mathrm{KDy}
$$


where $y$ is the column player's optimal strategy in the game $I-(1 / \mathrm{k}) \mathrm{D}$ and $\mathrm{K}$ is as in Equation 14. Since $\operatorname{det}\left(\mathrm{I}-\frac{1}{\mathrm{k}} \mathrm{D}\right)=0$, the value of the game $I-\frac{1}{\mathrm{k}} \mathrm{D}$ is zero. For $r<1 / k$, we had $(I-r D) y=. v$; hence, in the limit we have $\left(I-\frac{1}{k} D\right) y=0$. But this means that $\mathrm{Dy}=\mathrm{ky}$ so that $\mathrm{y}$ is a characteristic vector of $\mathrm{D}$ associated with $\mathrm{k}$. We conclude that the dominance method and the Wei-Kendall method agree when $r=1 / \mathrm{k}$. These results are summarized in the following theorem.

THEOREM 6. - For $\mathrm{r}=1 / \mathrm{k}$ both the dominance method and the Wei-Kendall method assign as a score vector $H$ where $H$ is a constant and $y$ is:

(a) a characteristic vector of $\mathrm{D}$ associated with its principal characteristic value $\mathrm{k}$, and (b) an optimal strategy for the column player in the game I - $\frac{1}{\mathrm{l}} \mathrm{D}$.

A question that the author has been unable to settle is the following: Is the ordering given by the dominance method for $0<\mathrm{r}<1 / \mathrm{k}$ the same as it gives for $r=1 / \mathrm{k}$ ? In all the examples computed this has been the case, but no proof has as yet been found. If the answer is yes, then it would be sufficient merely to solve thë gåní in Equation 15 in order to get the unique ranking. 


\section{CHAPTER II}

\section{THE GENERAL CASE FOR PAIRWISE REDUNDANT RELATIONS}

\section{A. More on Order Relations}

Some of the material in this paragraph is covered in more detail in Section 1-5 of Unit II of Reference 11. A binary order relation $\mathbf{R}$ that is reflexive and transitive (see Chapter I, Section A) is called a weak ordering. Let us define a relation $E$ so that $A_{i} E A_{j}$ means " $A_{i} \dot{R} A_{j}$ and $A_{j} R_{i}$ "; then it can be shown that $E$ is an equivalence relation; that is, it is reflexive, symmetric, and transitive. Let $U$ be the set of equivalence classes of $A$ determined by $E$. We can also define a preference relation $P$ where $A_{i} P A_{j}$ means " $A_{i} R A_{j}$ and it is false that $A_{j} R A_{i} . " \bar{P}$ is reflexive and transitive. This preference relation induces a partial order $P^{\circ}$ on the set $U$ of equivalence classes. Thus if $X$ and $Y$ belong to $U, .$. then $X P^{\circ} Y$ if and only if $A_{i} P A_{j}$ for all $A_{i}$ in $X$ and all $A_{j}$ in $Y . P^{\circ}$ is also irreflexive and transitive.

In our case, we define $A_{i} R A_{j}$ to mean " $A_{i}$ can dominate $A_{j}$ in a certain number of stages." We shall state this verbally as, $A_{i}$ is comparable to $A_{j}$. By definition, we shall say that $A_{i} R A_{i}$ for all $i$; that is, each member is comparable to himself. By definiton, $R$ is reflexive and transitive and hence is a weak ordering relation. The corresponding equivalence relation $E$ is defined as " $A_{i} R A_{j}$ and $A_{j} R A_{i}$." Verbally, we say that $A_{i} E A_{j}$ if and only if $A_{i}$ and $A_{j}$ are mutually comparable: Let $U$ be the set of equivalence classes defined by $E$. The corresponding preference relation $P$ is defined verbally as $A_{i} P A_{j}$; that is, $A_{i}$ is preferred to $A_{j}$ if $A_{i}$ is comparable to $A_{j}$, but $A_{j}$ is not comparable to $A_{i}$. Finally, the induced partial ordering $P^{\circ}$ on the equivalence classes is $\mathrm{XP}^{\circ} \mathrm{Y}$ if equivalence class $\mathrm{X}$ is preferred to $\mathrm{Y}$; that is every element in $\mathrm{X}$ is preferred to every element in $\mathrm{Y}$.

In Section 2, we shall show that the induced partial ordering $P^{0}$ of equivalence classes is actually a linear ordering in the case of a pairwise redundant ordering relation. Then we show that the matrix of an equivalence class is regular, except in the case that the class contains one, two, or three elements. A ranking is then obtained as follows: Elements in different equivalence classes are ordered by the relation $P$ and elements in the same equivalence class are ordered by one of the ranking procedures described in Part $I$. We thus obtain a complete ranking in nearly all cases. The cases for which the method fails, namely those in which one or more members are tied, are discussed further in Chapter III, Section B.

\section{B. Equivalence Classes and Their Partial Ordering}

Here we carry out the program outlined at the end of the last section.

THEOREM 7. - - If $\gg$ is a pairwise redundant ordering, then the partial ordering $P^{0}$ of the set $U$ of equivalence classes is a linear ordering. 
PROOF. - - Let $X$ and $Y$ be two equivalence classes in $U$, and let $A_{i}$ be any element of $X$ and $A_{j}$ be any element of $Y$. Since the relation $\gg$ is pairwise redundant, we have either $A_{i} \gg A_{j}$ or $A_{j} \gg A_{i}$. Suppose it is the former. Then $A_{i} \gg A_{k}$ for every $A_{k}$ in $Y$, since otherwise $A_{k}$ would belong to $X$. Hence we conclude that $X P^{\circ} \mathrm{Y}$. Since every pair of equivalence classes is comparable under $P^{\circ}$ and since $P^{\circ}$ is transitive, we see that it gives a linear ordering of these classes, completing the proof.

In particular, Theorem 7 means that the maximal and the minimal equivalence classes under the partial order are unique. It also shows that if we can rank the elements in each equivalence class, we will then have a ranking of all the members of the set. A. We will see that it is not always possible to order elements within a given equivalence class (see Chapter III, Section B), but Theorem 9 below shows that the methods of Part I can be extended to make as complete a ranking as is possible within such equivalence classes.

DEFINITION. - - Let $X$ be an equivalence class and let $D_{X}$ be the dominance matrix that the members of $X$ define. We shall call $D_{X}$ the dominance matrix associated with $\mathrm{X}$.

Obviously $\mathrm{D}_{\mathbf{X}}$ is the submatrix of $\mathrm{D}$ obtained by taking only those rows and columns corresponding to members of the equivalence class $X$. If $X$ is a unit class, that is, consists of one member, then the matrix $D_{X}$ is the $1 \times 1$ matrix with entry zero. We next prove some results that show that $D_{X}$ is regular if there are at least four elements in the equivalence class $\mathrm{X}$.

LEMMA 1. -- Assume $\gg$ is a pairwise redundant ordering, and let $X$ be an equivalence class and $\mathrm{DX}_{\mathrm{X}}$ be its associated dominance matrix. If there is an entry, say the $i$, $j$ th entry, of $D_{X}^{m}$ that is positive for all sufficiently large powers of $D_{X}$, then $D_{X}$ is regular.

PROOF. - - If for all $m \geq M$ the $i, j$ th entry of $D X$ is positive, then it is possible to go from $A_{i}$ to $A_{j}$ in $M$ steps, $M+1$ steps, $M+2$ steps, etc. Let $N$ be the largest number of steps required to go from $A_{j}$ to any other element of $X$; such a number exists since it is possible to go from $A_{j}$ to every individual in $X$. Then it is possible to go from $A_{i}$ to every other state in $M+N$ or more steps so that the ith row of $D_{X}^{M+N}$ has all positive entries. Let $K$ be the largest number of steps required to go from any element in $X$ to $A_{i}$; then the ith column of $D \mathrm{M}+\mathrm{N}+\mathrm{K}$ has all positive entries since we can go from any state in $x$ to $A_{i}$ in $K$ or fewer steps, and we can go from $A_{i}$ to any other state in $M+N$ or more steps. Now the square of a matrix having all positive entries in its ith row and its ith column will have all its entries positive. Therefore $D_{X}^{2(M+N+K)}$ and all higher powers of $D_{X}$ are positive and $D_{X}$ is regular.

THEOREM 8. .... Let $\gg$ be a pairwise redundant dominance relation. If $X$ is an equivalence class containing four or more elements, and $D_{X}$ is its associated matrix, then $\mathrm{D}_{\mathbf{X}}$ is regular. 
PROOF. - - The graph of a pairwise redundant dominance relation defined on a set of $n$ individuals means a set of $n$ points in the plane with each pair of points being connected by a line segment, and on each line segment one or two arrows indicating the direction of dominance. There are only four pairwise complete dominance relations on a set of four elements. The graphs of three of these are shown in Fig. 1; the fourth dominance relation is simply the transitive ordering of the four individuals. If $X$ is an equivalence class containing exactly four members then its graph must be that of Fig. 1(a) since the other two graphs

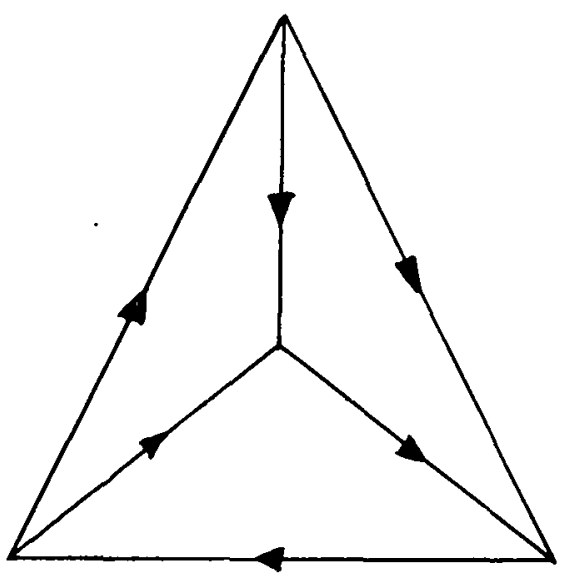

(a)

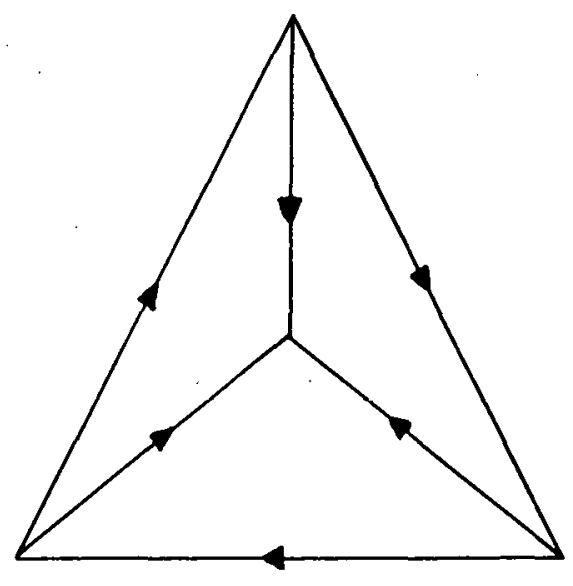

(b)

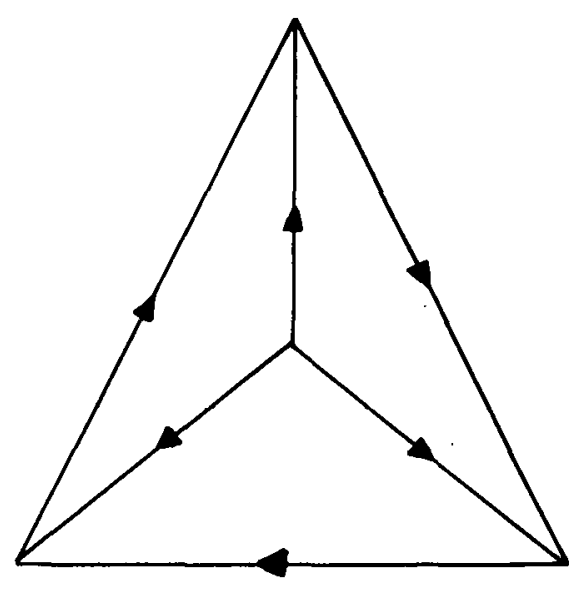

(c)

Figure 1

(and that of the transitive relation) contain two or more equivalence classes. By direct computation it can be shown that $\mathrm{D}_{\mathrm{X}}^{9}>0$ if $\mathrm{X}$ is the equivalence class whose graph is shown in Fig. 1(a). If $X$ is an equivalence class containing five or more members then it contains an intransitive triple, that is, a triple with $A_{i} \gg A_{j} \gg A_{k} \gg A_{i}$. This holds because all triples can be transitive if and only if the dominance relation itself is transitive. Consider such a triple and then consider the relation of each of the other elements of $X$ to the triple. If there is an element whose relation to the triple is as in Fig. 1(a), then the submatrix of $\mathrm{DX}$ defined by these four elements is positive for ninth and greater powers so that Lemma 1 implies that $\mathrm{DX}$ is regular. If all the other elements of $X$ have to the intransitive triple the relation depicted in Fig. 1(b) or all have the relation of Fig. 1(c), Then these elements cannot belong to the same equivalence class. Therefore, if none of the elements are related as in Fig. 1(a) then there is at least one as in Fig. 1(b) and at least one as in Fig. 1(c). Moreover, it is possible to choose one of each type so that the resulting graph is as in Fig. 2; otherwise these elements cannot all belong to the same equivalence class. But now we see that the elements $A_{1}, A_{2}, A_{4}$, and $A_{5}$ have a graph equivalent to that of Fig. 1(a) so that again Lemma 1 applies and $D_{X}$ is regular. 


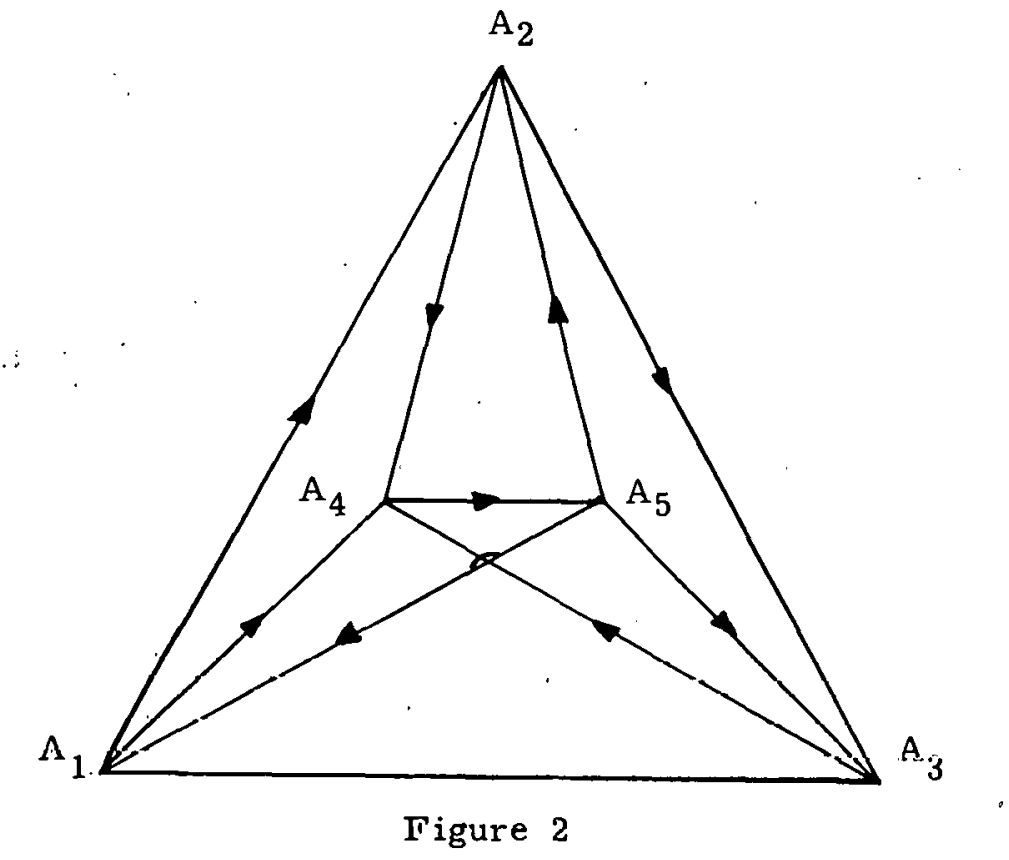

THEOREM 9. -- If $\gg$ is a pairwise redundant ordering, $X$ is an equivalence class and $D_{X}$ is not regular, then there are three possibilities:

(a) $\mathrm{X}$ contains only one element.

(b) $\mathrm{X}$ contains two elements $\mathrm{A}$ and $\mathrm{B}$, ordered $\mathrm{A} \gg \mathrm{B} \gg \mathrm{A}$.

(c) $\mathrm{X}$ contains three elements, A, B, and C, ordered A $\gg$ B $\gg$ C $\gg$ A.

PROOF. - - By Theorem 8, $X$ cannot have more than three elements. (a) If $X$ has one element, then, as already observed, its matrix is simply the number 0 and is not regular. (b) If $X$ has two elements $A$ and $B$, then necessarily $A \gg B$ and $B \gg A$. since they must be mutually comparable. It is easy to check that $D X$ is not regular. (c) If $X$ has three elements $A, B$, and $C$, then necessarily $A \gg B ~ \gg C ~ \gg A$, so that they belong to the same equivalence class. With oniy these relations among them it is easy to show that $D_{X}$ is not regular. With any further relations it is easy to show that $D_{X}$ is regular, proving (c).

Observe that Case (b) is a two-way tie and Case (c) is a three-way tie. It is evident that there is no way of breaking such ties with only the information given. Other kinds of ties are discussed in Chapter III, Section B.

The way in which the results of this section can be used to extend the ranking methods given in Chapter $I$ is now obvious. Given a pairwise redundant ordering relation on the members of a set A, first separate the members into equivalence classes by means of the mutually comparable relation. To compare members of different equivalence classes, use the linear ordering relation $\mathbf{P}^{\circ}$. To compare members of the same equivalence class, use one of the scoring methods of Chapter I. In some cases (as, for example, in (b) and (c) of Theorem 9) ties will have to be allowed. In practice, such ties are rare and a complete ranking will usually be obtained. Ties may be broken by submitting the tied individuals to another judge, (see Chapter III, Section A). 


\title{
CHAPTER III
}

\author{
EXTENSIONS, EXAMPLES, AND COMPARISONS
}

\section{A. Multiple Judgments.}

We can think of the binary relation discussed in Chapters I and II as being given by a single judg $\epsilon$ who compares the members of the set. Suppose, as is often the case, that the members of a given set are rated by two or more judges. For example, we may be interested in testing which of a number of production processes is best for a certain job, and we apply a battery of tests to compare each pair of processes. Kendall (Reference 3 ) has proposed adding the dominance matrices of each judge and then using his scoring method on the sum matrix. This result could also be interpreted as the result of a single judge who can cast a variable number of votes for members of a pair, instead of just one vote. Here we wish to propose a modification of Kendall's procedure. Suppose we have $m$ judges, and each judge has a dominance matrix $D_{1}, \ldots, D_{m}$ that indicates how he rates the members of the set. Suppose, in turn, that we can rate the judges according to how important we feel their judgments are; let the weight we give to judge $k$ be $\lambda_{k}$, where $\lambda_{k}$ is a positive real number. Then we form the matrix,

$$
F=\lambda_{1} D_{1}+\lambda_{2} D_{2}+\ldots+\lambda_{m} D_{m}=\sum_{k} \lambda_{k} D_{k} \text {. }
$$

The resulting matrix will not, in general, have only 0 and 1 entries, but it will have zeros on its main diagonal. However, we shall say that $A_{i}$ is comparable to $A_{j}$ if there is a nonzero entry in the $i, j$ th entry of $F$; by definition we say that $A_{i}$ is comparable to $A_{i}$, and then extend the notion of comparability by making it transitive. As in Chapter II, we define equivalence classes and then use either one of the judging methods of Chapter I to set up an order among member of an equivalence class. The theorems previously proved hold; in some cases a slight modification is necessary. The matrix $F$ will usually define fewer equivalence classes and there will be fewer ties than for the relation defined by any individual matrix $D_{k}$. Again the matrix $F$ may be interpreted as the matrix of a single judge who can cast a variable number of votes for the members of each pair. An example of a multiple judgment is worked out in the next section.

\section{B. Examples}

1. Comparison of the Scoring Methods of Chapter I. -- Consider the dominance matrix (20) for the graph of Fig. 1(a). It is the matrix of the only pairwise complete dominance

$$
\mathrm{D}=\left(\begin{array}{llll}
0 & 1 & 1 & 0 \\
0 & 0 & 1 & 1 \\
0 & 0 & 0 & 1 \\
1 & 0 & 0 & 0
\end{array}\right)
$$


relation on four objects whose mutually comparable relation defines a single equivalence class. The characteristic equation of $D$ is $x^{4}-2 x-1=0$ and its principal characteristic root is 1.395. Table I shows the scores obtained from the dominance method, written as deviations from the mean score divided by $s_{1}$. The last score, corresponding to $r=0.717=1 / 1.395$, is also the score given by the Wei-Kendall scoring method. The scores always place the members in the same order although the actual. numerical scores vary with $r$. The exact numbers obtained depend upon the weight given to higher order dominances.

TABLE I

\begin{tabular}{|c|c|c|c|c|}
\hline $\mathrm{r}$ & $s_{1}$ & $\mathrm{~s}_{2}$ & $\mathrm{~s}_{3}$ & $\mathrm{~s}_{4}$ \\
\hline 0.100 & 1.000 & 0.834 & -1.008 & -0.826 \\
\hline 0.200 & 1.000 & 0.718 & -1.028 & -0.690 \\
\hline 0.300 & 1.000 & 0.635 & -1.054 & -0.581 \\
\hline 0.400 & 1.000 & 0.574 & -1.085 & -0.489 \\
\hline 0.500 & 1.000 & 0.529 & -1.118 & -0.412 \\
\hline 0.600 & 1.000 & 0.496 & -1.151 & -0.345 \\
\hline 0.700 & 1.000 & 0.471 & -1.185 & -0.285 \\
\hline 0.717 & 1.000 & 0.467 & -1.191 & -0.276 \\
\hline
\end{tabular}

2. Multiple Judging. - - Suppose that two different judges of four individuals rate them according to the dominance matrices in (21).

$$
\mathrm{D}_{1}=\left(\begin{array}{llll}
0 & 1 & 1 & 1 \\
0 & 0 & 0 & 1 \\
0 & 1 & 0 & 0 \\
0 & 0 & 1 & 0
\end{array}\right) \quad \mathrm{D}_{2}=\left(\begin{array}{llll}
0 & 1 & 0 & 1 \\
0 & 0 & 1 & 1 \\
1 & 0 & 0 & 0 \\
0 & 0 & 1 & 0
\end{array}\right) .
$$

If the judges are given weights of one each, the $F$ matrix is

$$
F=\left(\begin{array}{llll}
0 & 2 & 1 & 2 \\
0 & 0 & 1 & 2 \\
1 & 1 & 0 & 0 \\
0 & 0 & 2 & 0
\end{array}\right)
$$

To find the scores of the individuals we solve the game $I-\frac{1}{4} F$ (first checking that $1 / 4$ is less than the principal characteristic root of $F$ ) and find that the optimal strategy for the column player has components $s_{1}=0.349, s_{2}=0.233, s_{3}=0.225$, and $s_{4}=0.193$. Hence the composite judgment of the two judges ranks the individuals $\mathrm{A}_{1} \mathrm{~A}_{2} \mathrm{~A}_{3} \mathrm{~A}_{4}$. 
3. Ties. - - As observed earlier there sometimes are ties among members of the same equivalence class which cannot be broken by the scoring methods developed. Figure $3(\mathrm{a})$ shows a three-way tie whose dominance matrix is not regular (cf. Theorem 9); Fig 3(b) shows a five-way tie whose dominance matrix is necessarily regular (cf. Theorem 8). The reader

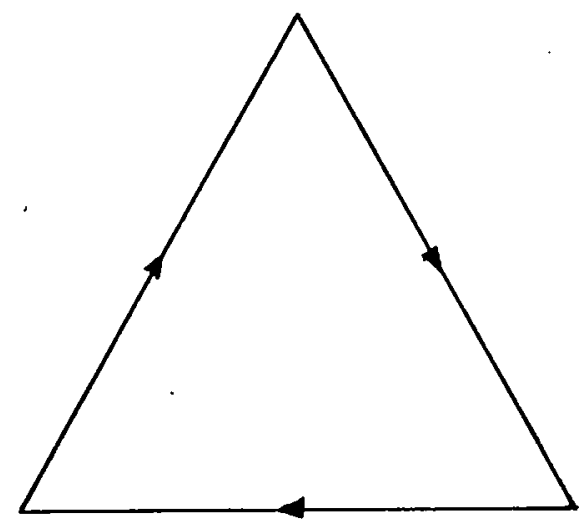

(a)

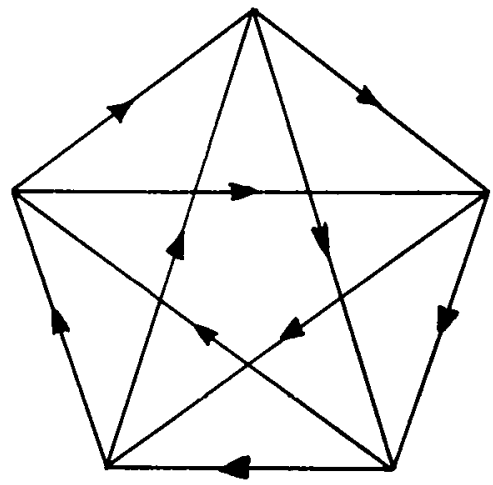

(b)

Figure 3

may wish to construct analogous seven-way and larger odd-number ties. It can be shown that a four-way tie is impossible with a pair-wise complete relation. It is probably true that evennumber ties greater than four are also impossible in this case.

Figure 4(a) shows the graph of a dominance relation among five individuals that contains a three-way tie $\left(A_{3}, A_{4}\right.$, and $A_{5}$ are tied. The graph in Fig. $4(b)$ is that of a dominance relation among six individuals. By considering the equations satisfied by a characteristic matrix of the corresponding $D$ matrix, or those satisfied by an optimal strategy vector, we can show that $A_{4}$ and $A_{5}$ are tied by both the scoring methods of Part $I$. This is clear graphically since they each dominate the other and also dominate the same individuals. There seems to be no reasonable way to break these ties with only the information given. But if we could submit the same individuals to another judge, it is quite likely that the combination of their two judgments would break the tie.

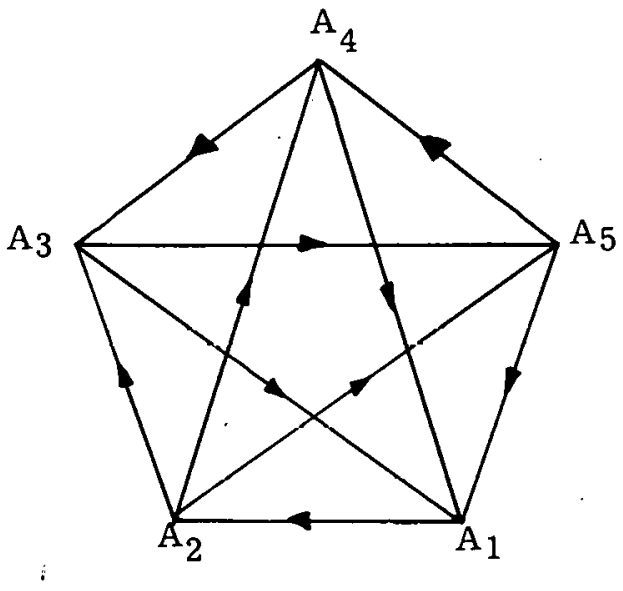

(a)

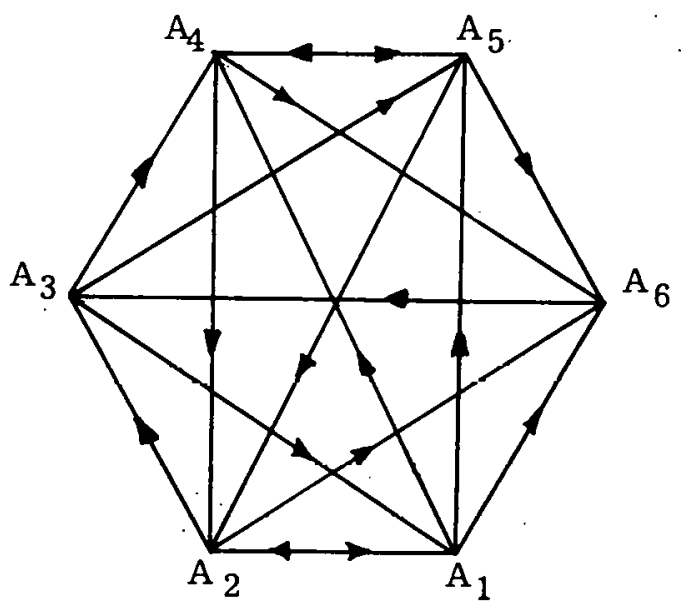

(b)

Figure 4

These examples show some of the difficulties that can arise in judging problems. 


\section{Comparisons and Extensions}

The methods discussed here do not depend upon the assumption of an underlying psychological scale as do those of Thurstone-Mosteller (References 4, 5, and 6). Nor do they depend upon an underlying statistical model, as.does Scheffe's model (Reference 7). In Reference 6, Mosteller has applied his model to the analysis of baseball data where certainly the judging method is impersonal and there is no psychological scale. On the other hand, the Wei-Kendall method, together with other methods, was used by Bliss, Greenwood, and White (Reference 1) on a case in which tasters were asked to compare pairs of various kinds of applesauce. That case seems very well suited to the assumption of a psychological scale. From six judgments by each of 25 tasters they obtained the following matrix, in which the entry at the $i, j$ th spot indicates the number of times $i$ was preferred to $j$.

$$
\begin{aligned}
& 1 \\
& 2 \\
& 3
\end{aligned} \quad\left(\begin{array}{rrrr}
1 & 2 & 3 & 4 \\
0 & 81 & 95 & 103 \\
69 & 0 & 81 & 81 \\
55 & 69 & 0 & 78 \\
47 & 69 & 72 & 0
\end{array}\right)
$$

In Table II we reproduce some of the data from Reference 1 that compares the various methods of ranking the kinds of applesauce, together with the row player's optimal strategy vector in the game I - $\frac{1}{500} \mathrm{D}$. They are written as deviations from the mean score divided by the largest score. Observe that all methods give the same ranking, but that the actual

\section{TABLE II}

$\begin{array}{lcccc} & 1 & 2 & 3 & 4 \\ \text { Mean normal deviate, Mosteller } & 1.000 & 0.108 & -0.409 & -0.699 \\ \text { Degree of preference, ranks, Scheffe } & 1.000 & 0.044 & -0.294 & -0.750 \\ \text { Optimal strategy in game I - (1/500)D } & 1.000 & 0.130 & -0.435 & -0.696\end{array}$

numerical scores differ somewhat. Bliss, et al, state a preference for the ThurstuneMosteller technique because of computational ease, and probably also because it is a familiar kind of computation.

The solution of a four-by-four game by trial-and-error methods is not difficult and can be done with a desk computer in less than 2 hours. Methods of computing the solutions of very large games have been coded for electronic computers and would allow the application of the dominance method to large problems.

It should be remarked that only a reinterpretation is necessary in order to modify the order relation discussed here to one that expresses degree of preference in the sense of

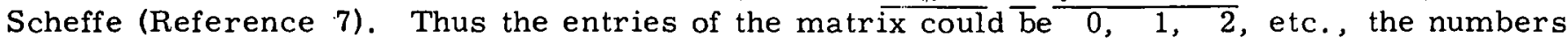
indicating how much one member of the pair is preferred over the other. 
A final remark should be made which has relevance to footnote 15 of Reference 7 . Although the various models for obtaining the ranking are derived from widely differing rationales--psychological, statistical, sociological, or graph-theoretic--the final mathematical model in many cases has been applied with impunity to situations in which the rationale of the model does not apply. The fact that the results of the various methods are in close agreement indicates, perhaps, that they each extract approximately the same information from the data gathered, regardless of how they were developed. 


\section{REFERENCES FOR PART ONE}

1. Bliss, C. I., Greenwood, M. L., and White; E. S.

A Rankit Analysis of Paired Comparisons for Measuring the Effect of Sprays on Flavor, Biometrics, 12, (1956), pp 381-403.

2. Fisher, R. A., and Yates, F.

Statistical Tables for Biological, Agricultural and Medical Research

4th edition, Oliver and Boyd, London, 1953.

3. Kendall, M. G.

Further Contributions to the Theory of Paired Comparisons,

Biometrics, 11 (1955), pp 43-62.

4. Mosteller, F.

Remarks on the Method of Paired Comparisons: I. The Least Squares Solution Assuming Equal Standard Deviations and Equal Correlations,

Psychometrika, 16 (1951), pp 3-9.

5. Mosteller, F.

Remarks on the Method of Paired Comparisons: II. The Effect of an Aberrant Standard Deviation When Equal Standard Deviations and Equal Correlations are Assumed,

Psychometrika, 16 (1951), pp 203-206.

6. Mosteller, F.

Remarks on the Method of Paired Comparisons: III. A Test of Significance for Paired

Comparisons When Equal.Standard Deviations and Equal Correlations are Assumed,

Psychometrika, 16 (1951), pp 207-218.

7. Scheffe, H.

An Analysis of Variance for Paired Comparisons,

Journal of the American Statistical Association, 47 (1952), pp 381-400.

8. Luce, D. R., and Perry, A. D.

A Method of Matrix Analysis of Group Structure,

Psychometrika, 14 (1949), pp 95-116.

9. Ross, I. C., and Harary, F.

On the Determination of Redundancies in Sociometric Chains, Psychometrika, 17 (1952), pp 195-208.

10. Kemeny, J. G., Snell, J. L., and Thompson, G. L.

Introduction to Finite Mathematics,

Prentice-Hall, New York, 1957. 
11. Modern Mathematical Methods and Models, Volume II, to be published by the Committee on the Undergraduate Program of the Mathematical Association of America.

12. Debreu, G., and Herstein, I. N.

Nonnegative Square Matrices,

Econometrica, 21 (1953), pp 597-607.

13. Wielandt, $\mathrm{H}$.

Unzerlegbare, Nicht Negative Matrizen,

Mathematische Zeithschrift, 52 (1950), p 642.

14. McKinsey, J. C. C.

Introduction to the Theory of Games,

McGraw-Hill, 1952, 371 pp. 
THIS PAGE

\section{WAS INTENTIONALLY LEFT BLANK}




\section{PART TWO \\ NOTES ON FINITE MARKOV CHAINS}

\section{CHAPTER IV}

\section{REGULAR · MARKOV CHAINS}

A probability vector (row or column) is a vector with nonnegative components whose sum is one. A stochastic matrix is a nonnegative square matrix whose row sums are equal to one.

Let $S$ be a finite set containing $n$ elements called states. Let $p(0)$ be a probability vector, the initial probability vector. Its components give the probabilities of being at each of the states initially. Let $P=\left\|p_{i j}\right\|$ be an $n \times n$ stochastic matrix, the matrix of transition probabilities, whose entries. $\mathrm{p}_{\mathrm{ij}}$ give the probabilities of going from state $\mathrm{i}$ to state j. A finite Markov chain is a stochastic (time-dependent) process defined by the initial probability vector $\mathrm{p}(0)$ and the transition matrix $P$.

Let $p_{1 j}(m)$ be the probability of being at state $j$ after $m$ steps, given that the process started at state i. It can be shown that $p(m)=p(0) P(m)=p(0) P m$, where $P m$ is the ordinary matrix power, $P(m)=\left\|p_{i j}^{(m)}\right\|$, and $p(m)$ is the probability of being in each of the states after $m$ steps.

A stochastic matrix is said to be regular if some power of the matrix has all positive components.

THEOREM 1. - - If $P$ is a regular stochastic matrix, then

(a) The powers $\mathrm{Pm}$ approach a matrix $\mathrm{T}$.

(b) Each row of $T$ is the same probability vector $t$.

(c) The components of $t$ are positive.

The proof depends upon the Frobenius theorem (see Theorem 1 of Part One).

THEOREM 2. - - If $\mathrm{P}, \mathrm{T}$ and $\mathrm{t}$ are as in Theorem 1, then

(a) If $\mathrm{p}$ is any probability vector, $\mathrm{pPm} \rightarrow \mathrm{t}$.

(b) The vector $t$ satisfies $t P=t$, i.e., it is a fixed vector of $P$; $t$ is the unique such fixed probability vector. 
This theorem has the following important interpretation: Call a Markov chain regular if its transition matrix $P$ is regular. Then $p^{(m)}=p^{(0)} P m \rightarrow t$, so that, after, a large number of steps, the probability of being in state $j$ is very nearly $t_{j}$ regardless of the initial probabilities $p(0)$. The quantity $t_{j}$ is the limiting probability of being in state $j$.

(For examples see Chapter 15 of Reference 1, or Chapters V and VII of Reference 2.)

THEOREM 3. -- For a regular Markov chain, the average time it takes to return to a state, having once been there, is the reciprocal of the limiting probability of being in that state.

Theorems 2 and 3 answer the two most important questions concerning regular Markov chains. (Proofs may be found on pages 221 and 323 of Reference 2.) 


\section{CHAPTER V}

\section{NONREGULAR CHAINS}

\section{A. The Classification of States}

The classification here is similar to the classification of members of a set discussed in the judging problem (Part One of this monograph). Let us say that state i can communicate with state $j$ in one step if $p_{i j}>0$. Similarly, state $i$ can communicate with state $j$ $\overline{i n} m$ steps if there is a chain of statcs $i=i_{1}, i_{2}, \ldots, i_{m}=j$ such that $i_{k}$ can communicate with $i_{k+1}$ in one step. By definition we shall say that state $i$ can communicate with itself (in zero steps, say). The communication relation, which we symbolize by $C$, is then reflexive and transitive--therefore a weak ordering. The related equivalence relation $E$ is

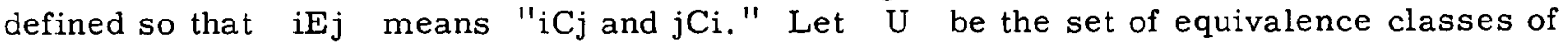
states determined by $\mathrm{E}$. The related preference relation $\mathrm{P}$ is defined as $\mathrm{Mi} \mathrm{Pj}$ if $\mathrm{iCj}$ and not jCi." Preference relation $P$ induces a partial order $P^{\circ}$ on the set $U$ of equivalence classes. Thus, if $X$ and $Y$ belong to $U$ then $X P^{\circ} Y$ if and only if $i P j$ for all in $X$ and all $\mathrm{j}$ in $\mathrm{Y}$.

EXAMPLE. -- Consider the Markov chain with transition matrix:

$$
\left(\begin{array}{lllll}
1 / 4 & 1 / 4 & 1 / 3 & 0 & 1 / 6 \\
1 / 3 & 1 / 6 & 1 / 6 & 1 / 3 & 0 \\
0 & 0 & 1 & 0 & 0 \\
0 & 0 & 0 & 1 / 4 & 3 / 4 \\
0 & 0 & 0 & 2 / 3 & 1 / 3
\end{array}\right)
$$

Here are three equivalence classes, one containing States 1 and 2, one containing State 3 , and one containing States 4 and 5 . They are ordered by $\mathrm{P}^{\circ}$ according to the graph below:

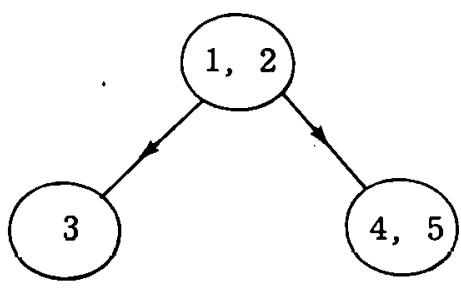

\section{B. Definitions}

DEFINITION. - - The minimal equivalence classes under the partial ordering $P^{\circ}$ are called ergodic classes. The states in ergodic classes are ergodic states. If an ergodic class contains exactly one state then that state is called an absorbing state. All states that are not ergodic are called transient states. 
In the example above, 1 and 2 are transient states; 3,4 and 5 are ergodic states; and 3 is an absorbing state.

DEFINITION. - - If $X$ is an equivalence class with $k$ states, then $P_{X}$ is the $\mathrm{kxk}$ submatrix of $\mathrm{P}$ whose entries have indices of states in $\mathrm{X}$.

LEMMA 1. - - If $\mathrm{X}$ is an ergodic class, then $\mathrm{P}_{\mathrm{X}}$ is a stochastic matrix.

DEFINITION. - - An ergodic class $X$ is regular if $P_{X}$ is a regular matrix. An ergodic class that is not regular is periodic. States in ergodic classes are regular or periodic according to the type of their class.

Obviously, an absorbing state is regular.

(The notation given here differs somewhat from that used by Feller in Reference 1.

DEFINITION. -- An absorbing Markov chain is one for which each ergodic state is absorbing. In such a chain it is always possible to go from any state to an absorbing state (in one or more steps).

EXAMPLE. - - The Peter-Paul matching-pennies game is an absorbing Mlarkov chain. Its matrix, for the case when the total fortune is three pennies, is given below. The states. are Peter's fortune.

$$
\begin{aligned}
& 0 \\
& 1 \\
& 3
\end{aligned}\left(\begin{array}{cccc}
1 & 0 & 0 & 0 \\
1 / 2 & 0 & 1 / 2 & 0 \\
0 & 1 / 2 & 0 & 1 / 2 \\
0 & 0 & 0 & 1
\end{array}\right)
$$

The behavior of $P_{X}$ for $X$ a regular ergodic class has been discussed previously. If $X$ is a periodic ergodic class, then there is a positive integer $p \geq 2$ such that if we "look at" the process only at times $p, 2 p, 3 p, \ldots$ then its behavior is like that of a - régular class. We shall content ourselves with thị sketchy discussion of periodic classes. (More information is available in Reference 1 ).

Regular and periodic classes act like absorbing classes since once the process enters such a class it never leaves it. If we want to know only what the chances are of it entering such a class and not what happens after that, we can replace such regular or periodic class by absorhing rlass (state). 'Iherefore the rest of the discussion will center on absorbing Markov chains.

\section{Absorbing Markov Chains}

Three important questions are to be answered for absorbing Markov chains.

(a) What is the probability that the process will end up in a given absorbing state?

(b) What is the average length of time for the process to reach some absorbing state?

(c) On the average, how many times will the process be in each transient state before final absorption? 
Contrary to the case of regular Markov chains, the answer to these questions may depend on the starting state.

Suppose there are $m$ absorbing states $i_{1}, i_{2}, \ldots, i_{m}$. Let $d_{j}\left(i_{k}\right)$ be the probability of being absorbed at state $i_{k}$ if the process starts at state $j$. Because it is impossible to leave an absorbing state, it is obvious that

$$
d_{i}\left(i_{k}\right)=0 \text { if } h \neq k \text {, }
$$

and

$$
d_{i_{k}}\left(i_{k}\right)=1
$$

Let $d\left(i_{k}\right)$ be the vector with components $d_{j}\left(i_{k}\right)$. From a state $j$ the process goes to state $u$ with probability $p_{j u}$ after which there is probability $d_{u}\left(i_{k}\right)$ of being absorbed at $i_{k}$; hence, $p_{j u} d_{u}\left(i_{k}\right)$ is the probability of going from $j$ to $i_{k}$ via state $u$. The sum of all these terms gives the total probabilily of going from $j$ to $i_{k}$. This can be written as a matrix product so that

$$
d\left(i_{k}\right)=P d\left(i_{k}\right)
$$

It can be shown that there is a unique probability vector solution to Equations 24-26. Note that the solution $d\left(i_{k}\right)$ is a fixed column probability vector of the transition matrix P. From the fact that the process must be absorbed it is easy to see that

$$
\sum_{k=1}^{m} d\left(i_{k}\right)=e .
$$

(Recall that $e$ is the $n$-component column vector each of whose entries is one.)

The components of the vector $d\left(i_{k}\right)$ give the answer to Question (a) above. Thus the $j$ th components of the vectors $d\left(i_{k}\right)$ give the probabilities of being absorbed in each of the absorbing states, given that the process started in state $j$.

EXAMPLE. - - In the Peter-Paul matching-pennies game above, it is easy to see that the two fixed vectors are

$$
\left(\begin{array}{c}
1 \\
2 / 3 \\
1 / 3 \\
0
\end{array}\right) \quad \text { and } \quad\left(\begin{array}{c}
0 \\
1 / 3 \\
2 / 3 \\
1
\end{array}\right)
$$

Thus, if Peter's fortune is one, he has $2 / 3$ chance of losing the game and $1 / 3$ chance of winning, and so forth. 
We next study Question (c) and develop results that will answer both Questions (b) and (c). If $i$ and $j$ are transient states, let $t_{i j}$ be the expected number of times the process will be in state $j$ given that it started in state $i$. Let us derive the relations that the $t_{i j}$ must satisfy.

From $i$ the process goes to state $\mathbf{k}$ with probability $p_{i k}$. If $\mathbf{k}$ is an absorbing state, then the process never goes to $j$; but if $k$ is transient then we expect it to go to $j$ an average of $t_{k j}$ times. We must therefore sum $p_{i k} t_{k j}$ over the transient states $k$. In case $i=j$, we must also add one to this sum since we started in $j$. To write these relations in matrix form let $Q$ be the submatrix of $P$ consisting of the entries whose indices are those of the transient states. Let $T=\left\|t_{i j}\right\|$. Then $T$ and $Q$ are of the same size and the above relations can be written

$$
T=Q T+T \text {. }
$$

This can be rewritten as

$$
(I-Q) T=I \text {. }
$$

It can be shown that the matrix $I-Q$ is nonsingular so that

$$
T=(I-Q)^{-1} \text {. }
$$

The entries of the matrix $(I-Q)=1$ give the answers to Question (c). They also give the answers to Question (b). If we want to know how many sleps the procece makes hefore absorption, given that it started at a transient state $i$, we observe that, before absorption, the process must always be in a transient state. If it started at $i$, it will be in each of the transient states the average number of times given by the entries of the ith row of $T$. Hence, the average number of times before absorption is the sum of the entries in the ith row. Thus the components of the vector $t$, where

$$
\mathrm{t}=\mathrm{Te}=(\mathrm{I}=\mathrm{Q})^{-1} \mathrm{e}
$$

provide the answers to Question (b).

EXAMPLE. - - In the Peter-Paul matching-pennies example above, we have

$$
\begin{aligned}
\mathrm{Q} & =\left(\begin{array}{cc}
0 & 1 / 2 \\
1 / 2 & 0
\end{array}\right), \\
\mathrm{I}-\mathrm{Q} & =\left(\begin{array}{cc}
1 & -1 / 2 \\
-1 / 2 & 1
\end{array}\right), \\
\mathrm{T}=(\mathrm{I}-\mathrm{Q})^{-1} & =\left(\begin{array}{cc}
4 / 3 & 2 / 3 \\
2 / 3 & 4 / 3
\end{array}\right), \text { and } \\
\mathrm{t} & =\left(\begin{array}{l}
2 \\
2
\end{array}\right) .
\end{aligned}
$$


From this we see that the average number of plays before the game ends is two, of which an average of $4 / 3$ of the plays are made in the starting state and $2 / 3$ in the other transient state. 


\section{CHAPTER VI}

\section{THE OPEN LEONTIEF MODEL *}

The Leontief input-output model considers an economy in which there are $r$ industries with the simplifying assumption that each industry produces exactly one kind of good. We regard the natural factors of production such as land, timber, minerals, etc, as free and do not consider them as entering into the cost of finished goods. In general, the industries are interconnected in the sense that each must buy a certain amount (positive or zero) of the other's products in order to run its industry. We define technological coefficients as follows: $a_{i j}$ is the dollar value of the amount of the output of industry $j$ that must be purchascd by industry $i$ in order that industry $i$ may produce $\$ 1$ worth of its own goods. Let $A$ be the $r \times r$ matrix with entries $a_{i j}$. By their definition the technological coefficients are nonnegative; hence, $A \geq 0$. (Here and elsewhere an equality or inequality relation between two vectors or matrices is assumed to hold if and only if it holds componentwise.)

It is easy to see that the sum of the $a_{i j}$, for $i$ fixed, gives the total value of the inputs needed by the ith industry in order to produce $\$ 1$ worth of its goods. If the ith industry is to be profitable, or at least to break even, this sum must be less than or equal to the value of its output; i.e., $a_{i 1}+a_{i 2}+\ldots+a_{i r} \leq 1$ for $i=1,2, \ldots, r$. For obvious reasons, we shall call the ith industry profitable if the strict inequality holds and profitless if the equality holds. We make the assumption that every industry is either profitable or profitless and thus rule out the possibility of unprofitable industries.

If we let $e$ be the $r$-component column vector each of whose entries is 1 , we can restate the above conditions as

$$
\begin{aligned}
A & \geq 0, \\
A e & \leq e .
\end{aligned}
$$

Having discussed the inputs of the industries, we next discuss their outputs. Let $\mathbf{x}_{\dot{j}}$ denote the monetary value of the output of the ith industry and let $x=\left(x_{1}, x_{2}, \ldots, x_{r}\right)$ be the row vector of outputs. Since the $i$ th industry needs an amount $x_{i} a_{i j}$ of llie output of the $j$ th industry, and this is true for $i, j=1,2, \ldots, r$, the vector of inputs needed by the industries is simply $x A$. Then the jth component of $x A$ gives the total value of the output that must be produced by the $j$ th industry in order to meet the interindustry demand for its product.

* This chapter reports on recent (unpublished) work by J. G. Kemeny, J. L. Snell, and G. L. Thompson. 
Assume that the economy supplies for consumption an amount. $h_{i}$ of the output of the ith industry. Let $h=\left(h_{1}, h_{2}, \ldots, h_{r}\right)$ be the row consumption vector. We shall require that

$$
h \geq 0
$$

that is, the consumption vector is nonnegative.

The requirement that the production vector of the economy be adjusted so that the interindustry needs as well as the consumption needs be fulfilled is now easy to write in vector form as

$$
x=x A+h .
$$

Rewriting Equation 35 as

$$
x(I-A)=h,
$$

we see that it is a set of $r$ simultaneous equations in $r$ unknowns.

To be economically meaningful, we must find nonnegative solutions to Equation 36 . Since the demand vector $h$ may be arbitarary, Equations 36 are in general nonhomogeneous and will have a solution if and only if the matrix I - A has an inverse. Moreover, the solutions to Equation 36 will be nonnegative for every $h$ if and only if (I - A)-1 has all nonnegative components. We must therefore search for necessary and sufficient conditions that the inverse of $I$. - A be nonnegative.

We will now imbed our model in a Markov chain, to be able to use Markov chain methods in proofs.

DEFINITION. - - By the Markov chain associated with an input-output model we shall mean a Markov chain $M$ with the following properties:

(a) The states of $M$ are the $r$ processes of the model plus one additional absorbing state $[$ the $(r+1)$ st state], called the banking state.

(b) The transition matrix $P$ of $M$ is defined as follows:

$$
\begin{aligned}
p_{i j} & =a_{i j} \text { for } i, j=1,2, \ldots, r \\
p_{i, r+1} & =1-\sum_{j=1}^{r} a_{i j}, \text { for } i=1, \ldots, r \\
p_{r}+1, j & =0, \text { for } j=1, \ldots, r \\
p_{r}+1, r+1 & =1 .
\end{aligned}
$$


The intuitive interpretation of $M$ is the following: If Industry $i$ receives a dollar for its use, then it spends it by buying $a_{i 1}$ from Industry 1 , $a_{i 2}$ from Industry 2 , etc., and $r{ }^{a}$ ir from Process $r$. The remainder of the dollar, if any - that is, the amount $1-\sum_{j=1}^{r} a_{i j}--$ is the profit, and we may think of it as being deposited in a bank. The fact that the last row of the transition matrix, the one corresponding to the banking state, has all zeros except for the one on the main diagonal, means that the bank represents an absorbing state. The bank gets money but does not spend it.

THEOREM 1. -- Let $A$ be a matrix satisfying Equations 32 and 33. A nonnegative solution to Equation 35 exists for every $h \geq 0$ if and only if the associated Markov chain $M$ is absorbing with the banking state as its only absorbing state.

\section{PROOF.}

Sufficiency. -- If $M$ is an absorbing Markuv chain with the banking statc as its only absorbing state, then $Q=A$ where $Q$ is the submatrix with indices corresponding to transient states. Also $T=(I-Q)^{-1}=(I-A)^{-1}$ is the matrix whose entries give the mean number of times the process is in each transient state. Since the mean number of times in a transient state is necessarily finite and nonnegative, the components of $(I-A)^{-1}$ are nonnegative, which is the desired conclusion.

Necessity. -- If $M$ is not an absorbing chain with only a single absorbing state, then there is another ergodic class whose indices are those of entries in A. But then (I - A) -1 cannot exist. If it did, it would have the interpretation as above; that is, its entries would give the mean number of times in each state, and the mean number of times the process is in an ergodic state is infinite. Hence $(I-A)^{-1}$ does not exist.

(A nonprobabilistic proof of the theorem can also be provided.) 


\section{CHAPTER VII \\ SEMIMARTINGALES OF MARKOV CHAINS}

A martingale relative to a stochastic process is a function on the states of the process such that the expected value of the function after one step is equal to the value of the function at that state. An upper (lower) semimartingale is a function on a state whose expectation after one step is greater (smaller) than the value of the function at the state. Here we shall assume that the stochastic process is an absorbing Markov chain.

Recall that an absorbing Markov chain is one all of whose ergodic states are absorbing states. Assume that there are $r$ absorbing states which we shall call boundary states. Let $\dot{B}$ be the set of boundary states. The remaining (transient) states will be called interior states. Assume there are $s$ interior states, and let $I$ be the set of interior states.

Let $P$ be the $(r+s) \times(r+s)$ transition matrix. A function on the states may be represented by a column vector $z$. Such $a \quad z$ is a martingale if $P z=z$; it is an upper semimartingale if $\mathrm{Pz} \geq \mathrm{z}$; and it is a lower semimartingale if $\mathrm{Pz} \leq \mathrm{z}$.

A one-person game interpretation makes these definitions clearer. If $z$ is a column vector, let $z_{i}$ be the value to the player of being at state $i$. Suppose he plays the following game: If he is at state $i \in B$, he receives the value $z_{i}$; if he is at a state $i \in I$, he may either accept the value $z_{i}$ or move to another state with the transition probabilities of the Markov chain. Then a martingale is a fair game, an upper semimartingale a favorable game, and a lower semimartingale an unfavorable game. Wc shall use this game interpretation to give intuitive proofs of certain results that follow.

We shall concentrate on nonnegative semimartingales. Suppose that nonnegative boundary values $v_{j}$ have been assigned to the boundary states $j \in B$. Let $U$ be the set of all nonnegative upper semimartingales $z$ with $z_{j}=v_{j}$ for $j \in B$. Thus $U$ is the set of all $z$ satisfying

$$
\begin{aligned}
\text { (a) } \quad P z \geq z, \\
\text { (b) } z \geq 0, \text { and } \\
\because \quad \text { (c) } z_{j}=v_{j} \text { for } j \in B .
\end{aligned}
$$

Similarly, $L$ is the set of all lower semimartingales, that is, vectors $z$ satisfying (b) and (c) and condition (a) with the inequality sign. reversed.

* This chapter summarizes some recent (unpublished) work of J. G. Kemeny and J. L. Snell. 
We shall develop a representation theorem for nonnegative semimartingales having the prescribed boundary values. Every such martingale can be represented as a combination of certain basic martingales.

Let $T$ be a subset of $I$, and denote by $P(T)$ the transition matrix obtained from $P$ by changing the states in $T$ into absorbing states. Let $Q(T)=\lim _{n \rightarrow \infty}[P(T)]^{n}$. The entry $q_{i j}(T)$ of $Q(T)$ represents the probability that, starting at state $\rightarrow \underset{i}{n}$, the process will reach state $j$ before reaching any element of $T$. Let $q_{j}(T)$ denote the jth column of $Q(T)$; then, since $Q(T)=P(T) \cdot Q(T)$, and since

$$
q_{i j}(T)=\left\{\begin{array}{cl}
\sum_{k} p_{i k} q_{k j}(T) & \text { if } i \notin T \\
0 & \text { if } i \in T
\end{array}\right.
$$

we see that

$$
P q_{j}(T) \geq q_{j}(T) \text { for } j \in B
$$

Thus, $q_{j}(T)$ for $j \in B$ is an upper semimartingale. Its value is one at $j$ and zero at all other absorbing states and at all states in $T$. Then the vector

$$
z(T)=\sum_{j=1}^{r} v_{j} q_{j}(T)
$$

io a nonnegntive upper semimartingale with the prescribed boundary values; hence $z(T) \in U$. We shall call $z(T)$ a basic upper semimartingale.

The game represented by the semimartinglae $z(T)$ is the following: If the player is at. a state $i$ in $B$, the game is stopped and he receives $z_{j}(T)$. If he is at a state $i$ in $T$, the game stops and he receives $\bar{z}_{i} \equiv 0$. If lie is at a otate if $T-T$, he may quit and receive $z_{i}$ or he may move to another state with the transitiun piubabilitico of the Marknv chain. This interpretation makes the following lemma obvious:

LEMMA 1. - - Let $\mathrm{T}_{1}$ and $\mathrm{T}_{2}$ be subsets of $\mathrm{I}$ with $\mathrm{T}_{1} \subseteq \mathrm{T}_{2}$. Then $z\left(T_{1}\right) \geq z\left(T_{2}\right)$.

EXAMPLE. - - Consider the Markov chain whose transition matrix is:

$$
P=\left(\begin{array}{cccc}
1 & 0 & 0 & 0 \\
0 & 1 & 0 & 0 \\
0 & 1 / 3 & 1 / 3 & 1 / 3 \\
1 / 2 & 0 & 1 / 4 & 1 / 4
\end{array}\right)
$$


The sets of states are $B=\{1,2\}$ and $I=\{3,4\}$. Assume that $v_{1}=2$ and $v(2)=1$. The basic semimartingales are

$$
\begin{gathered}
z(\phi)=\left(\begin{array}{c}
2 \\
1 \\
7 / 5 \\
9 / 5
\end{array}\right) \quad z(\{3\})=\left(\begin{array}{c}
2 \\
1 \\
0 \\
4 / 3
\end{array}\right) . \\
z(\{4\})=\left(\begin{array}{c}
2 \\
1 \\
1 / 2 \\
0
\end{array}\right) . \\
z(\{3,4\})=\left(\begin{array}{c}
2 \\
1 \\
0 \\
0
\end{array}\right) .
\end{gathered}
$$

It is clear that $z(\{3,4\})$ is also a martingale.

Lemma 1 partially orders the basic semimartingales. The maximal element in the partial ordering is $z(\phi)$ (where $\Phi$ is the empty set), and the minimal element is $z(I)$. It is easy to see that $z(\phi)$ is a martingale with the proper boundary values.

We now make a special assumption that is to hold for the rest of the discussion. It can be relaxed but we shall not go into the details.

HYPOTHESIS A. -- The boundary values $v_{j}$ for $j \in B$ are all positive and, for any interior state $i \in I$ there is at least one $j \in B$ so that $p_{i j}>0$. (In other words, onestep transition from any interior state to the boundary is always possible.)

LEMMA 2. -- If Hypothesis A holds, then

$$
\text { (i) }\{\mathrm{Pz}(\mathrm{T})\}_{\mathrm{i}}=\{\mathrm{z}(\mathrm{T})\}_{\mathrm{i}}>0 \text { for } \mathrm{i} \in \mathrm{I}-\mathrm{T} \text {, }
$$

and

(ii) $\{\mathrm{Pz}(\mathrm{T})\}_{\mathrm{i}}>\{\mathrm{z}(\mathrm{T})\}_{\mathrm{i}}=0$ for $\mathrm{i} \in \mathrm{T}$.

This can easily be proved from the game interpretation. Lemma 2 shows that for each $z(T)$ exactly one of the equalities (i) or (ii) holds.

LEMMA 3. - - Let $x_{1}, x_{2}, \ldots ; x_{n}$ be distinct nonnegative vectors, and let $w_{i}$ be the set of components of $x_{i}$ that are equal to zero. Assume that if $W_{i} \subseteq W_{k}$ then $\mathbf{x}_{\mathbf{i}} \geq \mathbf{x}_{\mathrm{k}}$. Then the vectors are convexly independent; that is, no one vector $\mathbf{x}_{\mathbf{i}}$ can be written in the form $x_{i}=\sum_{k \neq i} a_{k} x_{k}$ with $a_{k}>0$ and $\sum_{k} a_{k}=1$. 
PROOF. - - If the contrary holds, then a component of $x_{i}$ can be zero only if all the $x_{k}$ have this component zero. Hence $w_{i} \subseteq w_{k}$, and $x_{i} \geq x_{k}$ for all $k$. But his can' only be true if $x_{j}=x_{k}$ for all $k$, contrary to hypothesis.

DEFINITION. - - A convex $n$-dimensional polyhedron is cubic if in every $j$-dimensional face, for every $(j-1)$-dimensional subface there is a unique nonintersecting $(j-1)$ dimensional subface $(j=1, \ldots, n)$.

THEOREM 1. - - If Hypothesis $A$ is satisfied, then $U$ is a convex cubic polyhedron with $2^{S}$ corner points. These corner points are the $z(T)$ for $T \subseteq I$.

THEOREM 2 (The representation theorem). -- If Hypothesis A is satisfied, then U may be divided into $s$ ! simplices each of dimension $j$. These simplices intersect only in faces of lower dimension. Every upper semimartingale lies in a unique smallest simplex of dimension $j=0,1, \ldots, s$. These simplies are either the $s$-dimensional ones mentioned above, or their faces. Every upper semimartingale $z_{0}$ may be written uniquely as

$$
z_{0}=\sum_{j=0}^{k} a_{k} z\left(T_{j}\right), a_{j}>0, \sum a_{j}=1
$$

where $\mathrm{T}_{0} \subseteq \mathrm{T}_{1} \subseteq \ldots, \subseteq \mathrm{T}_{\mathrm{n}}$ and the $\mathrm{z}\left(\mathrm{T}_{\mathrm{j}}\right)$ are the corners of the unique smallest simplex containing $\mathrm{z}_{0}$.

This theorem can be extended to the case where Hypothesis A does not hold. It can also be extended to give a representation theorem for lower semimartingales. Application of these results to sequential games, statistical decision functions, and harmonic function theory is possible.

EXAMPLE. -- In the example above, the third and fourth components of the hasic semimartingales can be plotted as in Fig. 5 .

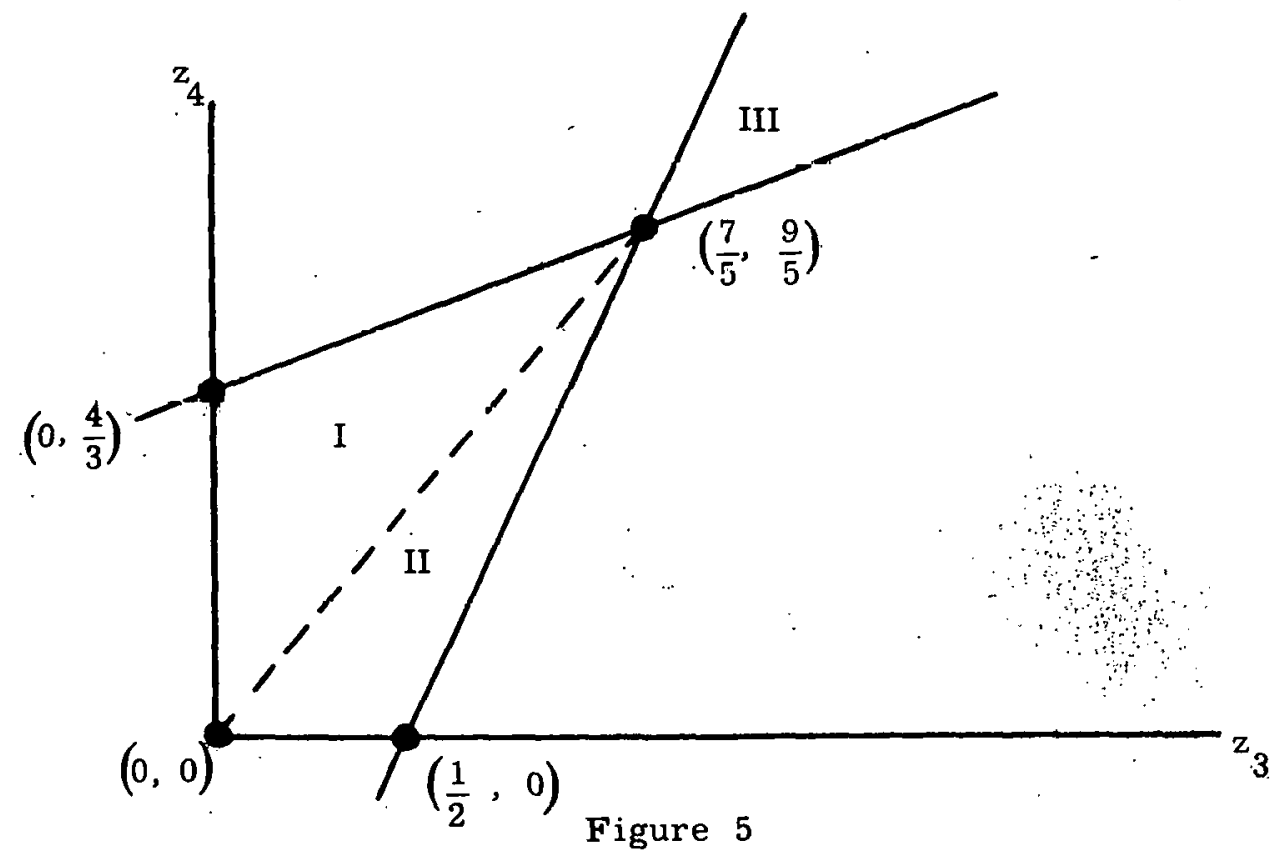


The four darkened points are the basic semimartingales, and I and II are the two simplices. Area III is the set of lower semimartingales. The point $(7 / 5,9 / 5)$ is both an upper and a lower semimartingale; hence, is a martingale. 


\section{REFERENCES FOR PART TWO}

1. Feller, William

An Introduction to Probability Theory and its Applications, Wiley, New York, 1957.

2. Kemeny, J. G., Snell, J. L., and Thompson, G. L. Introduction to Finite Mathematics, Prentice-Hall, New York, 1957. 
PART THREE

NOTES ON GAME .THEORY

CHAPTER VIII

MATRIX GAMES

\section{A. Introduction}

If $G$ is a real-valued $m \times n$ matrix, it can be considered a two-person matrix game between the row player $R$ and the column player $C$. Player $R$ chooses a row index $i$, $C$ chooses a column index $j$, and then $C$ pays an amount $g_{i j}$ to $R$. (If $g_{i j}$ is negative, then it represents a positive payment from $R$ to $C$. )

A probability. vector is a nonnegative vector, the sum of whose components is one.

Mixed strategies for the players are: for $\mathbf{R}$, an $\mathrm{m}$-component probability vector $\mathrm{p}$; and for $C$, an n-component probability vector $q$. A solution to the game consists of a real number $v$, the value of the game, and optimal strategy vectors $p^{0}$ for $R$ and $q^{\circ}$ for $C$ that satisfy

$$
\mathrm{p}^{\circ} \mathrm{Gq} \geq \mathrm{v} \geq \mathrm{pGq}
$$

for all $p$ and $q$. The principal (minmax) theorem of game theory states: Every matrix game has a solution. Two proofs of it are given in Section B.

The following facts may be establis̀ned (cf. Chapter VI of Reference 1).

(a) The value of a game is unique.

(b) $\mathrm{v}=\mathrm{p}^{\circ} \mathrm{Gq}^{\circ}$, hence $\mathrm{v}$ is an expected value.

(c) The value $v$ is the most $R$ can assure himself and the least $C$ can assure himself.

(d) A matrix game is strictly determined if there is an entry $g_{i j}$ in $G$ that is the minimum entry in the ith row and the maximum entry in the jth column. A strictly determined game may be solved by pure strategies, i.e., strategies that choose a row or column with probability one.

(e) The sets of optimal strategies for the players are convex, closed, polyhedral sets. 
(f) Let $E$ be the $m \times n$ matrix, each of whose entries is equal to one. Let $h$ be a positive number and $k$ be any number. Then the sets of optimal strategies in the games $G$ and $h G+k E$ are identical. If the value of $G$ is $v$, then the value of $h G+k E$ is hv $+k$. By this transformation, which can be interpreted as a monetary scale change plus a side payment, any matrix game can be changed to a fair game, that is, one with value zero, and with matrix entries having absolute value less than one. (Such a change is convenient for putting games on machines.)

\section{B. Proofs of the Main Theorem}

The minmax theorem for matrix games is true when the matrix entries are from an ordered field (such as the rationals) as well as when they are real. This observation was due to $\mathrm{H}$. Weyl. Actually the theorem has been extended in another direction to continuous games on the equare and nn generalized spaces of analysis. We shall present two proofs of the original theorem, one analytic and one algebraic. Each will depend on resulte in the literature.

1. The first proof valid for a real matrix $G$ is due to $J$. Nash (see Reference 2, $p$ 288). Let $\lambda_{i}$ and $\mu_{j}$ be pure strategies of the players for $i=1, \ldots, m$ and $j=1, \ldots, n$. If $P$ and $Q$ are the sets of mixed strategies for the players, consider $S=P \times Q$. For $S=(p, q)$, a point in $S$ defines continuous functions:

$$
\begin{aligned}
& r_{i}(s)=\max \left(0, \lambda_{i} G q-p G q\right) \\
& c_{j}(s)=\max \left(0, p G q-p G \mu_{j}\right) .
\end{aligned}
$$

Then we define a modified strategy vector $\dot{s}^{\prime}=\left(p^{\prime}, q^{\prime}\right)$ as follows:

$$
\begin{aligned}
& p_{i}(s)=\frac{p_{i}+r_{i}(s)}{1+\sum_{k} r_{k}(s)} \\
& q_{j}(s)=\frac{q_{j}+c_{j}(s)}{1+\sum_{k} c_{k}(s)} .
\end{aligned}
$$

We thus have a continuous mapping $T$ of $S$ into itself. The Brouwer fixed-point theorem now implies that there is at least one fixed point of the mapping, i.e., a vector $s$ such that $s^{\prime}=s$.

We must show that such a fixed point yields a pair of optimal strategy vectors for the game. If $\mathbf{s}=(p, q)$ is a fixed point, consider the vector $p$. Some of the components of $p$ are positive and among the positive ones there must be one, say the ith one, that is "least profitable" so that $\lambda_{i} G q \leq p G q$. This makes $r_{i}(s)=0$. Now $p_{i}>0$, and $p$ is fixed under the mapping so that the weight put on the ith row must not decrease under $T$. Since $r_{i}(s)=0$, this means that the denominator of the first quotient above must equal the one. But that means. $\sum r_{k}(s)=0$; i.e., $r_{k}(s)=0$; hence, $\lambda_{k} G q \leq p G q$ for $k=1, \ldots, m$. In the same way, $p G q \leq p G \mu$ for $j=1, \ldots, n$. From these two inequalities it is clear that $\mathrm{v}=\mathrm{pGq}$, and $\mathrm{p}$ and $\mathrm{q}$ are optimal strategies. 
2. The second proof, valid for a matrix $G$ with entries in an ordered field, is due to Gale, Kuhn, and Tucker (Reference 3, p 84) and depends on a result of Stiemke (Reference 4).

A matrix game $G$ is symmetric if $G$ is skew-symmetric;i.e., $g_{i j}=-g_{j i}$. An optimal strategy for one player is optimal for the other in a symmetric game. The value of a symmetric game is zero. Every game $G$ can be symmetrized by replacing it by the square symmetric game,

$$
S=\left(\begin{array}{ccc}
0 & G & -1 \\
-G^{t r} & 0 & 1 \\
1 & -1 & 0
\end{array}\right),
$$

where $G^{\text {tr }}$ is the transpose of $G$. The solutions of $G$ may be deduced from the solutions of $S$ (see Reference 3, p 83).

STIEMKE'S THEOREM. -- Let $A$ be an $m \times n$ matrix. Then, either $u A \geq 0$ (proper inequality for somc component) for some vector $u$, or else $A v=0$ for some vector $v>0$.

If $\mathrm{S}$ is an $\mathrm{n} \times \mathrm{n}$ skew-symmetric game, apply Stiemke's result to the matrix $(\mathrm{S}, \mathrm{I})$, where $\mathrm{I}$ is the $\mathrm{n} \times \mathrm{n}$ identity matrix. Then, either there exists a $\mathrm{u} \geq 0$ such that $u S \geq 0$, or there exists a $v>0$ with $S v<0$. Since $S$ is skew symmetric and $m=n$, we see that $S v<0$ implies $v^{\prime} S=-v^{\prime} S^{\prime}>0$, so that the second alternative cannot happen. If we normalize the $u \geq 0$ obtained from the first alternative so that $\sum u_{i}=1$, then we have found an optimal strategy for the game $S$.

\section{Other Theorems}

1. The Strong Minmax Theorem. -- Although the minmax theorem, proved above, gives the existence of solutions to matrix games, a stronger version of it is frequently useful.

THEOREM (The strong minmax theorem). -- If $G$ is a matrix game, there exist optimal strategy vectors $\mathrm{p}^{*}$ and $\mathrm{q}^{*}$ such that

$$
\left\{p^{*} G\right\}_{j}>0 \text { if and only if } q_{j}=0 \text {, }
$$

or equivalently

$$
\left\{p^{*} G\right\}_{j}-0 \text { if and only if } q_{j}>0 \text {, }
$$

and

$$
\left\{\mathrm{Gq}^{*}\right\}_{i}>0 \text { if and only if } \mathrm{p}_{\mathrm{i}}=0
$$

or equivalently

$$
\left\{\mathrm{crq}^{*}\right\}_{\mathrm{i}}=0 \text { if and only if } \mathrm{p}_{\mathrm{i}}>0 \text {. }
$$

Reference 5 contains a good proof of this theorem by A. W. Tucker. 
2. Dimension Theorems. -- Because the optimal strategy spaces of the players are determined by the same matrix, it is reasonable that there should be a connection between the dimensions of these spaces. The connection was found independently by Gale and Sherman and by Bohenblust, Karlin, and Shapley (References 6 and 7).

Call a pure strategy essential if it occurs with positive probability in some optimal mixed strategy, and call it superfluous if it does not. Denote by $e_{r}$ and $e_{c}$ ( $s_{r}$ and $s_{c}$ ) the number of essential (superfluous) pure strategies for the two players. Let $d_{r}$ and $d_{c}$ be the dimensions of the sets of optimal strategies for the players. Call a $(d-1)-$ dimensional bounding face of the set of optimal strategies for a player an interior face, if it does not lie on a $(d-1)$-dimensional face of the simplex of all optimal strategies for that player. Let $f_{r}$ and $f_{c}$ be the number of interior strategies for each player (these numbers are defined to be zero if the optimal strategies are unique).

THEOREM. - - A pair of convex polyhedra are the sets of optimal strategies for an $\mathrm{m} \times \mathrm{n}$ game if and only if

$$
\begin{aligned}
& e_{r}-d_{r}=e_{c}-d_{c} \\
& f_{r^{2}} \leq s_{c} \text { and } f_{c} \leq s_{r} .
\end{aligned}
$$

3. Kernels. -- A basic optimal strategy is an extreme point of a set of optimal strategies. Each player has a finite number of basic optimal strategies. Because of geometric interpretations, basic optimal strategies are the intersections of hyperplanes and hence should have algebraic formulas. These were discovered by Shapley and Snow (see Reference 8 ).

If $A$ is an $h \times h$ matrix let $A^{*}$ be the transposed cofactor matrix of $A$, let $f$ be an $\mathrm{h}$-component row vector each of whose entries is one, and let $\mathrm{e}$ be an $\mathrm{h}$-component row vector each of whose entries is one.

THEOREM. - - A necessary and sufficient condition that $\mathrm{p}^{\circ}$ and $\mathrm{q}^{\circ}$ be basic optimal strategies in a matrix game $G$ is that there is an $h \times h$ square submatrix $A$ of $G$ such that the entries of $p^{\circ}$ and $q^{\circ}$ agree with the components of the vectors

$$
\begin{aligned}
& p^{*}=\frac{f A^{*}}{f A^{*} e} \\
& q^{*}=\frac{A{ }^{*} e}{f A^{*} e},
\end{aligned}
$$

when restricted to $A$, and the value $v$ of the game $G$ is given by

$$
v=\frac{\operatorname{det} A}{\mathrm{fA}^{*} \mathrm{e}} \text {. }
$$

This theorem gives a (not very practical) method of solving a matrix game. Thus, by trying out all possible square submatrices of $G$, we could eventually find a pair of basic optimal strategies by using these formulas. 
4. Linear Programming. - - Let $A$ be an $m \times n$ matrix, $b$ be an $m$-component column vector, and $\mathrm{c}$ an $\mathrm{n}$-component row vector, and consider the following two problems:

a. Minimum Problem. -- Determine a row vector $x$ that minimizes the linear form $x b$ subject to the inequalities $x \geq 0$ and $x A \geq c$.

b. Maximum Problem. -- Determine a column vector $y$ that maximizes the linear form cy subject to the inequalities $\mathrm{y} \geq 0$ and $\dot{A} y \leq b$.

These are the so-called "dual" problems of linear programming. The principal theorem 'of linear programming states that these problems either both have or both have not a solution, and when they do have a solution, the minimum value in a. equals the maximum values in $\mathrm{b}$.

The format of these two problems is reminiscent of the requirements for optimal strategies in a game. The following theorem shows that there is a close connection. It is due to Gale, Kuhn, and Tucker (see Reference 9, p 327).

THEOREM. - - The dual linear programs have a common solution if and only if the symmetric matrix game

$$
\left(\begin{array}{ccc}
0 & A & -b \\
-A^{\operatorname{tr}} & 0 & c^{\operatorname{tr}} \\
b^{\operatorname{tr}} & -c & 0
\end{array}\right)
$$

has an optimal mixed strategy vector whose last component is positive.

\section{PROOF.}

Sufficiency. -- Let ( $x, y^{\text {tr }}$, d) be such a solution, where $x$ is a row vector, $y$ a column vector, and $d$ a positive constant. Then

$$
-y^{t r} A^{t r}+d b^{t r} \geq 0 \text { or } A\left(\frac{1}{d} y\right) \leq b \text {, }
$$

and

$$
x A-d c \geq 0 \quad \text { or }\left(\frac{1}{d} x\right) A \geq c
$$

and

$$
-x b+y^{\operatorname{tr}} e^{\operatorname{tr}} \geq 0 \quad \text { or } \quad x b \leq c y .
$$

From the first two inequalities we deduce $x b \geq \frac{1}{d}(x A y) \geq c y$, and hence $x b=c y$. Therefore $\frac{1}{d} x$ and $\frac{1}{d} y$ provide the solutions to the dual linear programs. 
Necessity. -- Let $x$ and $y$ be solutions to the dual linear programs; then $x \geq 0$, $x A \geq c, y \geq 0, \quad A y \geq b$, and $x b=c y$. Consider the vector ( $x, y+1)$. If we divide it by the positive constant $\lambda=\sum x_{i}+\sum y_{j}+1$, it is easy to show that it is an optimal strategy in the above matrix game, and that its last component is positive.

\section{Computational Methods}

Many methods have been proposed for the computation of solutions to matrix games. The most widely used such method is the simplex method which we will not discuss here (cf. Reference 9, p 359). Instead we shall discuss the fictitious play method.

The fictitious play method of solving games was invented by G. Brown (cf. Reference 9 , p. 374) and its convergence established by J. Robinson (cf. Reference 10, p 296). Because it involves only comparisons of numbers and additions, it is particularly well suited to highspeed machine computation even though its convergence is probably not very rapid. It can be modified so that it converges more rapidly. Here we present a brief description of the method, together with its application to a simple example.

Let $A$ be $m \times n$ matrix considered as a two-person zero-sum.game. We shall denote the $i$ th row of $A$ by $A_{i}$ and the $j$ th column by $A_{j}$. A marginal vector system $(U, V)$ for $A$ shall mean a sequence $U(0), U(1), U(2), \ldots$ of $n$-dimensional vectors and a sequence $\mathrm{V}(0), \mathrm{V}(1), \mathrm{V}(2), \ldots$ of $\mathrm{m}$-dimensional vector satisfying the fulluwing conditions:

(a) The minimum component of $U(0)=$ the maximum component of $V(0)$. (For example we could always choose $\mathrm{U}(0)$ and $\mathrm{V}(0)$ as being zero vectors. Except for this condition, these initial vectors are arbitrary.)

(b) $U(t+1)=U(t)+\cdot A_{i}$.

(c) $V(t+1)=V(t)+A_{j}$.

The indices $i$ and $j$ satisfy:

(d) $i$ is the index of a maximal component of $V(t)$.

(e) $j$ is the index of a minimal component of $U(t)$.

The above collection of rules defines simultaneous fictitious play. Alternate fictitious play is derived from the above by replacing (e) by

(e') $j$ is the index of a minimal component of $U(t+1)$.

It has been suggested that alternate fictitious play converges rnore rapidly than simultaneous.

J. Robinson (Reference 10) has proved the following theorem.

THEOREM. - - If (U, V) is a vector system for a matrix game $A$, then

$$
\lim _{t \rightarrow \infty} \frac{\min U(t)}{t}=\lim _{t \rightarrow \infty} \frac{\max V(t)}{t}=v .
$$


This theorem gives no clue as to how to compute optimal strategies. However, it is obvious that an approximate optimal strategy can be computed by remembering how of ten each row or each column has been added during the course of the fictitious play and dividing by the number of iterations.

EXAMPLE. - - Consider the nonstrictly determined game whose matrix is

$$
\left(\begin{array}{ll}
1 & 3 \\
4 & 2
\end{array}\right)
$$

The alternate fictitious play computation is summarized in the tableau below:

\begin{tabular}{|c|c|c|c|c|c|c|c|c|c|c|c|}
\hline & & & $V(0)$ & $V(1)$ & $\mathrm{V}(2)$ & $V(3)$ & & & & & $V(8)$ \\
\hline & 1 & 3 & 0 & 1 & 4 & 7 & 10 & 13 & 16 & 17 & 20 \\
\hline & 4 & 2 & 0 & 4 & 6 & 8 & 10 & 12 & 14 & 18 & 20 \\
\hline $\mathrm{U}(0)$ & 0 & 0 & $j i$ & 1 & 2 & 2 & 2 & 2 & 2 & 1 & 2 \\
\hline $\mathrm{U}(1)$ & 4 & 2 & 2 & & & & & & & & \\
\hline $\mathrm{U}(2)$ & 8 & 4 & 2 & & & & & & & & \\
\hline$U(3)$ & 12 & 6 & 2 & & & & & & & & \\
\hline & 13 & 9 & 1 & & & & & & & & \\
\hline & 14 & 12 & 1 & & & & & & & & \\
\hline & 15 & 15 & 1 & . & & & & & & & \\
\hline & 19 & 17 & 2 & & & & & & & & \\
\hline $\mathrm{U}(8)$ & 20 & 20 & 1 & & & & & & & . & \\
\hline
\end{tabular}

The exact solution was obtained after eight steps and is

$$
\begin{aligned}
& \mathrm{p}^{\circ}=\left(\frac{1}{2}, \frac{1}{2}\right) \\
& \mathrm{q}^{\circ}=\left(\begin{array}{c}
\frac{1}{4} \\
\frac{3}{4}
\end{array}\right) \\
& \mathrm{v}=\frac{20}{8}=\frac{5}{2} .
\end{aligned}
$$


Observe that the exact solution for $\mathrm{C}$ was obtained in four steps, and the exact solution for $\mathbf{R}$ was obtained in six steps, although the process moved away from these solutions as it went on. Such "cycling" phenomena are common in this method and can be taken advantage of. Kemeny and Thompson have a modified fictitious play procedure for the IBM 704 that does exactly that. 


\section{CHAPTER IX \\ THE EFFECT OF PSYCHOLOGICAL ATTITUDES ON THE OUTCOME OF GAMES ${ }^{*}$}

\section{A. Characterization of Strategy-Preserving Utility Functions}

Let $G$ be an $m \times n$ matrix game with matrix $\left\|g_{i j}\right\|$; we define val[G] to be the value of $G$, and $X[G]$ to be the set of optimal strategies for the first player in $G$. $A$ utility function $f$ shall mean a real-valued (nonconstant) monotone increasing function. If $f$ is a utility function and $G$ a game, then by $f(G)$ we shall mean the matrix game whose matrix is $\left\|f\left(g_{i j}\right)\right\|$.

DEFINITION. - - We shall say that the utility function $f$ is strategy preserving if and only if for all matrix games $G$ and all real constants $h$ we have

$$
X[f(G+h E)]=X[f(G)],
$$

where $E$ is the $\mathrm{m} \times \mathrm{n}$ matrix all of whose entries are unity.

The intuitive interpretation of a strategy preserving utility function is the following: Suppose that the row player has a fortune of $h$ dollars at the time he is to play the game G; his fortune at the end of the game will be $g_{i j}+h$, where $g_{i j}$ is the actual payoff he receives from the game; the utility which he assigns to this outcome is $f\left(g_{i j}+h\right)$; then a strategypreserving utility function is such that every strategy optimal in the game $f(G+h E)$ is optimal in the game $f(G)$ and conversely; in other words, it is such that the way in which the row player plays a matrix game $G$ is independent of the state of his fortune when he plays it. The purpose of this section is to characterize such functions.

Two important types of utility functions are the linear and exponential ones given by

$$
f(x)= \begin{cases}a x+c, & a>0, \\ a e^{b x}+c, & a b>0 .\end{cases}
$$

Here the letters $a, b$, and $c$ indicate parameters and the conditions on the parameters are chosen so that $f^{\prime}(x)>0$ for all $x$.

* This chapter summarizes some recent work by J. G. Kemeny and G. L. Thompson published in Contributions to the Theory of Games, Vol. III, Annals of Mathematics Studies, Number 39, Princeton, 1957, pp 273-298. 
Several facts about elementary matrix game theory will be needed subsequently. First, we recall that adding the same constant to every entry in the matrix does not change the sets of optimal strategies, nor does the multiplication of every entry by the same positive constant. (These two facts are sufficient to show that linear utility functions are strategy-preserving.) Secondly, we recall that a $2 \times 2$ matrix game is nonstrictly determined if and only if either the inequalities

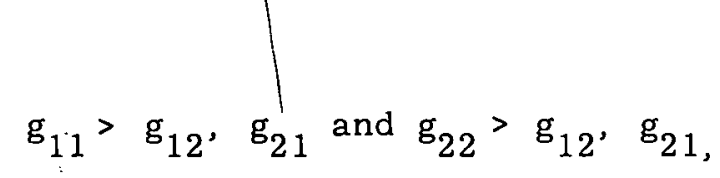

or the inequalities obtained from these by reversing the inequality signs, are satisfied. Finally, the first component of the optimal strategy for the row player in the $2 \times 2$ nonstrictly determined case is given by the formula

$$
x_{1}=\frac{g_{22}-g_{21}}{g_{11}-g_{12}-g_{21}+g_{22}} .
$$

LEMMA 1. -- If $f$ is a strategy-preserving utility function, then $f$ is differentiable and $f^{\prime}(x)>0$ for all $x$.

PROOF. - - If $f$ is monotone increasing but not strictly increasing (and nonconstant), then there exist real numbers $a, b$, and $h$, with $a<b$, so that $f(a)=f(b)$ and $f(a+h)<f(b+h)$. If we set $g_{11}=g_{22}=b$ and $g_{12}=g_{21}=a$, then every strategy for the row player is optimal in the game $f(G)$, but the game $f(G+h E)$ has a unique optimal strategy, so that $f$ is not strategy preserving.

If $f$ is monotone strictly increasing, thén, by a weil-known theorem it is differentldble almost everywhere. Hence $f$ has a derivative at two points, say at $a$ and $b$, where $\mathrm{a}>\mathrm{b}$. Set $\mathrm{g}_{11}=\mathrm{a}$ and $\mathrm{g}_{12}=\mathrm{g}_{21}=\mathrm{b}_{\text {; }}$. if $g_{? ?}=\mathrm{x}$ where $\mathrm{x}>\mathrm{b}$, then the $2 \times 2$ game $G=\left\|g_{i j}\right\|$ is nonstrictly determined. If $f$ is strategy-preserving, we have from Equation 49 that

$$
x_{1}=\frac{f(x)-f(b)}{f(a)-2 f(b)+f(x)}
$$

is the first component of the optimal strategy for the row player in the game $f(G)$; similarly,

$$
x_{1}^{*}=\frac{f(x+h)-f(b+h)}{f(a+h)-2 f(b+h)+f(x+h)}
$$

is the analogous quantity for the game $f(G+h E)$. For these two to be equal, we must have that

$$
\frac{f(x+h)-f(b+h)}{f(a+h)-f(b+h)}=\frac{f(x)-f(b)}{f(a)-f(b)} .
$$


Using the identity that if $d \neq f$, then $c / d=e / f$ implies $(c-e) /(d-f)=c / d$, we subtract corresponding terms on the right-hand side of this expression from those on the left. If we then divide numerator and denominator of the left-hand side of the resulting.expression by $h$, we obtain

$$
\frac{(1 / h)[f(x+h)-f(x)]-(1 / h[f(b+h)-f(b)]}{(1 / h)[f(a+h)-f(a)]-(1 / h[f(b+h)-f(b)]}=\frac{f(x)-f(b)}{f(a)-f(b)}
$$

which is true for all values of $h$. Letting $h$ tend to zero, and using the fact that $f$ has a derivative at $a$ and $b$, we see that $f$ has a derivative for all $x>b$. To show that. $f$ has a derivative for all $x \leq b$, we choose any two real numbers $c$ and $d$ where $c>d>b$, set $g_{11}=d, g_{12}=g_{21}=c$, and $g_{22}=x \leq b$, and use reasoning analogous to the above. Because $f$ is strictly increasing, $f^{\prime}(x)>0^{-}$for all $x$. This concludes the proof of the lemma.

THEOREM 1. - - A necessary and sufficient condition that a utility function should be strategy preserving is that it should be either linear or exponential as in Equation 48 ,

\section{PROOF .}

Sufficiency. - - We have already observed that linear functions are strategy-preserving. If $f$ is exponential, then, using the elementary facts about matrix games mentioned above, we have

$$
\begin{aligned}
x\left[\left\|f\left(g_{i j}+h\right)\right\|\right] & =x\left[\left\|a e^{b g_{i j}+b h}+c\right\|\right] \\
& =x\left[\left\|a e^{b g_{i j}} \cdot e^{b h}\right\|\right] \\
& =x\left[\left\|a e^{b g_{i j}}+c\right\|\right] \\
& =x\left[\left\|f\left(g_{i j}\right)\right\|\right] .
\end{aligned}
$$

Necessity. -- Let $G=g_{i j}$ be a nonstrictly determined $2 \times 2$ matrix game so that it has a unique optimal strategy for the row player. By the lemma, $f$ is strictly increasing, so that $f(G)$ and $f(G+h E)$ are also nonstrictly determined and have unique optimal strategies. By an analysis similar to that carried out in the lemma, for the first components of the optimal strategies for the row player in each of these games to be equal, we must have

$$
\frac{f\left(g_{22}+h\right)-f\left(g_{12}+h\right)}{f\left(g_{11}+h\right)-f\left(g_{21}+h\right)}=\frac{f\left(g_{22}\right)-f\left(g_{12}\right)}{f\left(g_{11}\right)-f\left(g_{21}\right)} .
$$


Observe that obvious linear solutions to this identity are linear functions of the form $f(x)=a x+c$, where we require $a>0$ to make $f^{\prime}(x)>0$. To find other solutions, use the identity mentioned in the proof of the lemma and subtract numerator and denominator of the right-hand side from the corresponding quantities on the left-hand side. Dividing through the resulting expression by $h$, letting $h$ tend to zero, and using the differentiability of $f$ as given by the lemma, we obtain the following differential equation

$$
\frac{f^{\prime}\left(g_{22}\right)-f^{\prime}\left(g_{12}\right)}{f^{\prime}\left(g_{11}\right)-f^{\prime}\left(g_{21}\right)}=\frac{f\left(g_{22}\right)-f\left(g_{12}\right)}{f\left(g_{11}\right)-f\left(g_{21}\right)} \text {, }
$$

which must be true for all nonstrictly determined games G. Observe that this equation becomes indeterminate for the linear solutions found above. After cross-multiplying the latter identity, setting the result equal to a constant and solving, we see that $f$ satisfies

$$
f^{\prime}(x)=b f(x)+d,
$$

for all $x$, where $b$ and $d$. are constants. The unique nonlinear solution of this differential equation is the exponential one

$$
f(x)=a e^{b x}+c,
$$

where $c=-d / b$. To satisfy the condition that $f^{\prime}(x)>0$, we require that $a b>0$, completing the proof of the theorem.

\section{B. Psychological Attitudes and Utility Functions}

In this section we shall discuss various types of psychological attitudes. For convenience we shall give names to them and leave to the reader the judgment of the suitability of these names.

By an attitude of the first kind, we shall mean one which depends only on the payoffs involved. Such attitudes can always be represented by means of a utility function. Attitudes of the second kind depend on factors other than the payoff and hence cannot be described by means of a utility function. All but one of our examples will be of utility functions of the first kind.

When discussing attitudes of the first kind, we shall usually require that $f(0)=0$ since, by a linear (strategy preserving) transformation, we can always make $f$ have this property. Figure 6 shows six types of continuous utility functions and two discontinuous ones.

The first such attitude is the "fair attitude" of a person who judges his utility to be directly proportional to the payoff. Since an additive constant can be ignored, we can think of the linear function $f(x)=x$.

Next there is the "reckless or gambler's attitude" of a person who concentrates on winning large sums. To him a large win looks even larger and a large loss is discounted. The result is a utility curve that is concave upward over its entire range. The exponential functions satisfying Equation 48 with $a>0$ are of this type. For example, the function $f(x)=e^{x}-1$ is such. 


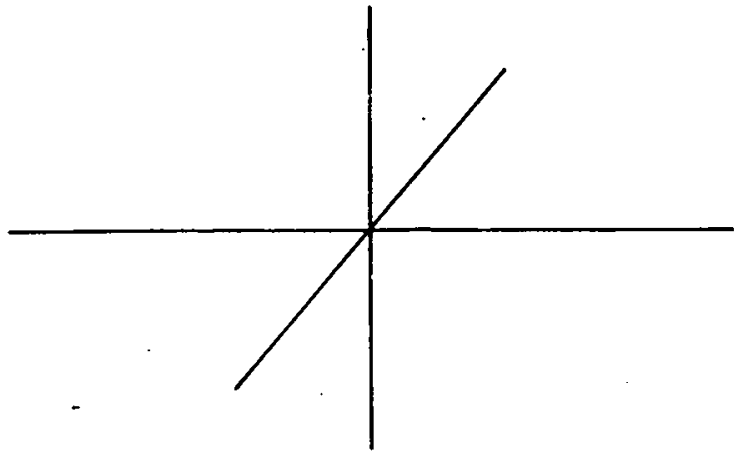

Fair

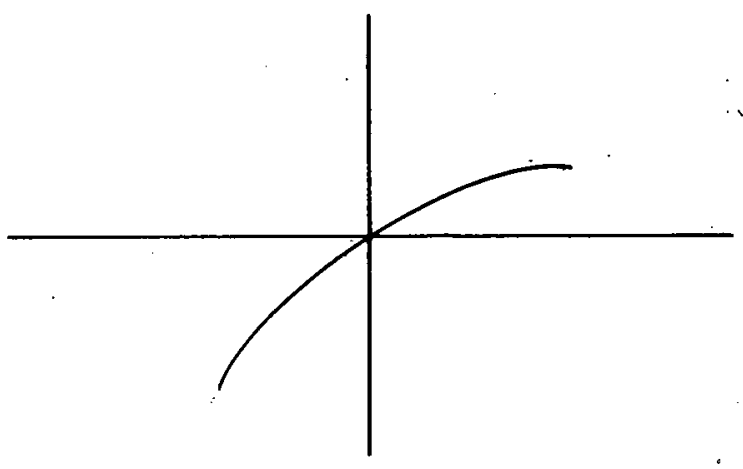

Cautious
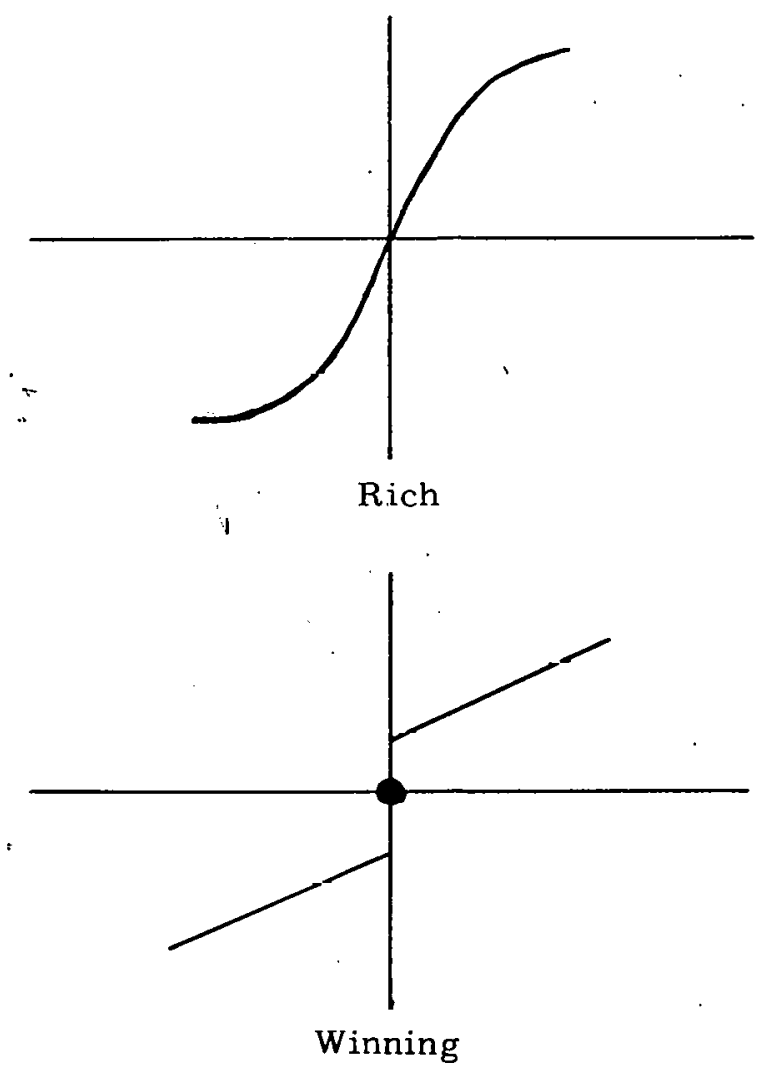
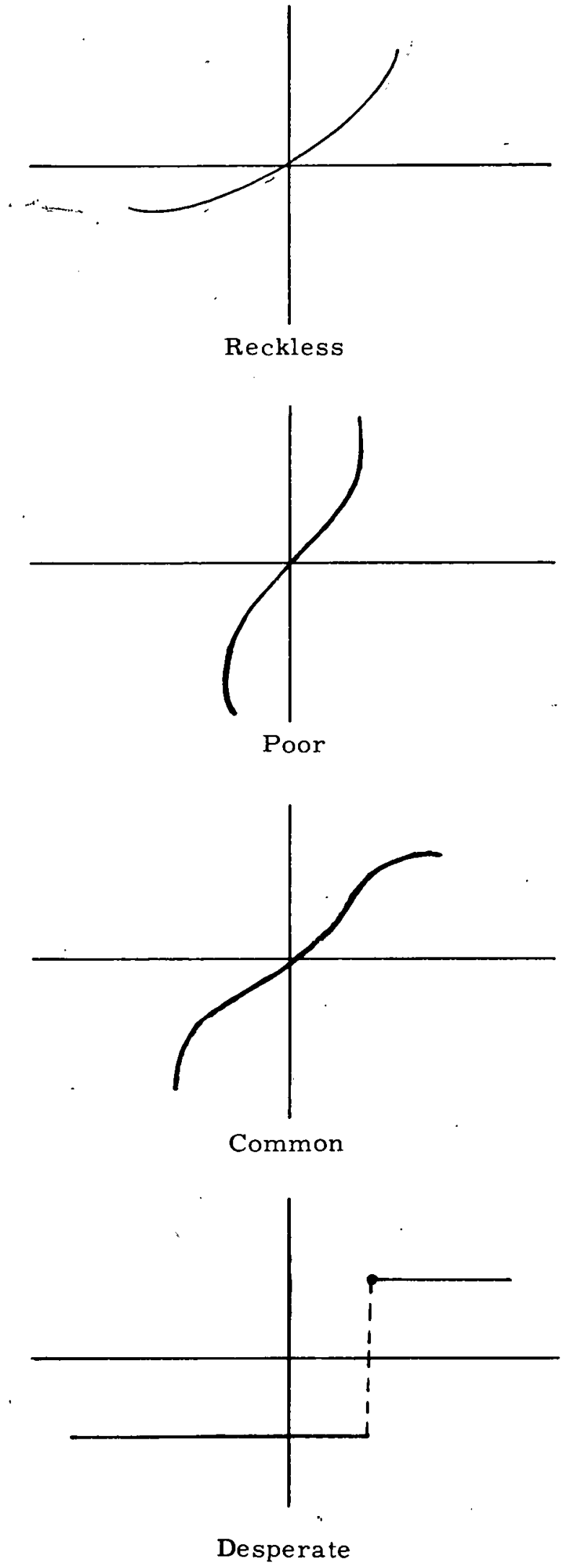

Figure 6

$34 \cdot 054$

57 
The "cautious attitude" is that of a person who concentrates on avoiding large losses. He exaggerates large losses and correspondingly discounts large wins. Thus his utility curve is convex downward over its entire range. The exponential functions satisfying Equation 48 with $a<0$ are of this type. For example, $f(x)=1-e^{-x}$ is such.

All functions satisfying Equation 48 represent one of these three types, hence we know that other attitudes must not satisfy Equation 48. It is interesting to note that utility curves similar to these three were observed in the Mosteller-Noges utility experiment. (See pages 386-395 of Reference 11.)

The "poor man's attitude" is that of a person for whom large sums, either positive or negative, are exaggerated. His utility curve is concave upward for positive $x$, and concave downward for negative $x$.

The "rich man's attitude" is that of a person to whom large sums, either positive or negative, are discounted. His utility curve is concave downward for positive $x$ and concave upward for negative $\mathbf{x}$.

The "common attitude" is that of a person who is reckless enough to play games in which the payoff entries are small, but when payoffs become large, he becomes cautious. Thus, for a range about zero his utility curve is convex upward, but it becomes concave downward as the absolute value of $x$ becomes large.

In addition to the continuous utility functions discussed above, a large number of discontinuous ones can also be distinguished. Here we mention just two interesting cases.

The "winning attitude" is that of a person who, besides considering how much he wins or loses, puts a positive premium on winning and a negative premium on losing. We shall assume that he has a fair attitude otherwise, so that his utility curve is the line $y=x$, with the positive half move up and the negative half moved down. By means of this utility function we are able to "explain" the paradoxial sequence game discussed in Section D, page 61 .

The "desperate attitude" is that of a person who must win a given sum of money. Any amount of money less than this is of no value to him and any amount in excess does not have greater value. His utility curve is a step function, positive from some sum on, and negative otherwise. (In this case we do not require that $f(0)=0$. )

The reader will doubtless be able to think of other intuitively interesting utility curves. The ones mentioned above are sufficient to discuss some interesting examples, several of which are described in the next two sections.

\section{Examples}

1. The Lottery Game. - - A player of the lottery game purchases from a banker a small chance of winning a large sum of money. Let $s$ be the price of the lottery ticket, let $K$ be the amount of the win, and let $p$ be the probability of win. The expectation of the banker is

$$
p(-K)+(1-p) s
$$


The banker will adjust $p$ so that his expectation is positive and hence so that the player's expectation is negative; i.e., he will choose

$$
\mathrm{p}<\frac{-\mathrm{s}}{\mathrm{K}-\mathrm{s}}<0
$$

Here $p$ is small since $K$ is large compared to $s$.

Suppose the player has a utility function $f$. He then evaluates his expected utility as

$$
E=p f(K)+(1-p) f(-s) \text {. }
$$

Let us assume that he will play only if his expected utility is positive, and will not play if it is negative or zero. Then we can make a list of typical decisions to play or not to play by the various players, depending upon their utility functions. It is to be emphasized that not all utility functions of a given type will make the same decision in the same situation; this will happen only when their shapes are sufficiently extreme.

$\underline{\text { Play }} \quad \underline{\text { Not play }}$

$\begin{array}{ll}\text { Reckless } & \text { Fair } \\ \text { Poor } & \text { Cautious } \\ \text { Common (K small) } & \text { Common (K large) } \\ \begin{array}{l}\text { Desperate (K greater than } \\ \text { discontinuity point) }\end{array} & \text { Desperate (K less than discontinuity } \\ & \text { Woint) } \\ & \text { Wichning }\end{array}$

The reader may check with his intuition as to whether the various decisions made on the basis of the utility functions are consistent with the names we have given them.

2. The Insurance Game. -- Here the player owns property worth $K$ dollars, and there is a small probability $\mathrm{p}$ of losing it by accidental means. The insurance company offers to pay the player $K$ dollars if the property is destroyed in return for a premium of $s$ dollars which the player pays to the company. The expectation of the insurance company is $(1-p) s+p(-K+s)$, and clearly they will adjust $s$ so that this expectation is negative; i.e., they will require that $s>p K$. A simple computation shows that this makes the player's monetary expectation negative. Suppose, however, the player has a utility function $f$; then his expected utility is $(1-p) f(-s)$ if he insures, and $p f(-K+s)$ if he does not insure; the difference of these two is

$$
D=f(-s)-p f(-K) \text {. }
$$

Let us assume that he will insure only if $D$ is positive. Again typical (but not necessary) decisions by persons having the above utility functions are as follows: 
Insure

Cautious

Poor

Common (if $\mathrm{K}$ large)
Not insure

Fair

Reckless

Common (if $\mathrm{K}$ small)

Rich

Winning

Desperate

Again the reader can check whether these decisions agree with his intuition. By adjusting $s$, the insurance company can appeal to people having different kinds of utility functions.

3. Colonel Blotto. - - The attitudes illustrated above were all of the first kind and con tinuous. Here we wish to illustrate a discontinuous attitude of the first kind and an attitude of the second kind.

That famed military leader, Colonel Blotto, has four divisions available and is contesting two mountain passes with an inferior enemy who has only three divisions. Assume that whichever side has the most divisions at a pass takes the pass and the opponent's divisions, except that in case of a tie neither side takes the pass and neither loses a division.

Blotto has three strategies corresponding to three possible ways of dividing his troops into two parts: $4+0,3+1$ and $2+2$. His opponent may divide his divisions in either of the two ways, $3+0$ or $2+1$. We assume that, having decided on the partitioning of the troops, the actual assignment to the passes is done at random.

Given a choice of a strategy by each player we can calculate the expected outcome. For example, if Blotto plays $3+1$ and his opponent plays $2+1$ we must distinguish two cases: (1) Blotto's three divisions go against the opponents ${ }^{\prime}$. two; then Blotto wins one pass and ties for the other; (2) Blotto's three divisions go against the opponents' one; then Blotto wins one pass and loses one. 'These possibilities are equally likely, so Blotto's payoff is $1 / 2$. Proceeding in this way we obtain the following payoff table:

\begin{tabular}{c|c|c|}
$4+0$ & $3+0$ & $2+1$ \\
$3+1$ \\
$3+2$
\end{tabular}$\quad$\begin{tabular}{|c|c|}
\hline $1 / 2$ & 0 \\
\hline $1 / 2$ & $1 / 2$ \\
\hline 0 & 1 \\
\hline
\end{tabular}

The resulting matrix game is strictly determined and Blotto should always play $3+1$ and his opponent should always play $3+0$. The value of the game to Blotto is $1 / 2$, indicating his numerical superiority. If both players play optimally, there is probability $1 / 2$ that Blotto will lose a division.

But now let us suppose that Blotto takes into account not only the number of passes that he can capture but also worries about the number of his men who are captured; in other words he has an attitude of the second kind. To be specific, assume that to Blotto a division lost is 
as bad as a pass lost. The resulting payoff table can be computed to be the following:

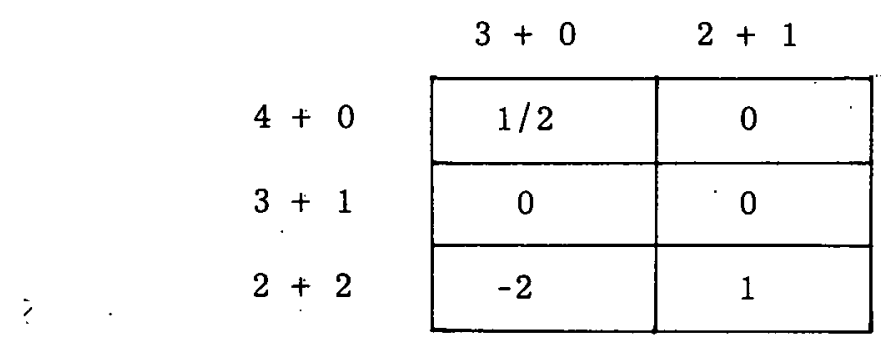

The solution to this game is that Blotto should play the first row with probability $6 / 7$ and the last row with probability $1 / 7$; his opponent should play the first column with probability $1 / 7$ and the second column with probability $6 / 7$. The value of the game to Blotto is $1 / 7$, indicating that his concern over loss of troops has decreased his expectation in the game. If both players play optimally, then Blotto's probability of losing a division is only $1 / 49$ as compared with probability $1 / 2$ in the preceding example. Thus his new strategy is successful in reducing the probability of troop loss.

As a final variant, assume that Blotto has the desperate attitude and wants at all costs to capture one pass. To be definite, assume that regardless of losses, he puts value one on capturing one or more passes and zero on not capturing a pass. It is easy to see that this makes all entries in the payoff table equal to one so that any strategy for Blotto is optimal. Thus the desperate attitude obscures strategic differences.

\section{A Sequence Game}

As a more complicated example of an application of utility functions, let us consider the game of matching pennies in which the players have agreed to quit after $\mathbf{N}$ plays, and in which the row player is given the option of quitting sooner if he wishes. According to ordinary game-theoretic arguments, this game is fair and hence a "rational" person should not be unwilling to accept the role of either player in the game. Yet most people would gladly play the role of the row player, and would refuse the role of the column player.

Let the game $G$ be given by the matrix

\begin{tabular}{|c|c|}
\hline 1 & -1 \\
\hline-1 & 1 \\
\hline 0 & 0 \\
\hline
\end{tabular}

where the row of zeros corresponds to the optional stop privilege of the row player. The rules of the game are: After the $N$ th play of $G$ the game stops; after fewer than $N$ plays of $G$ the game continues if the row player has chosen one of the first two rows of the matrix but stops if he has chosen the third row.

If the row player has the winning attitude given by the utility function

$$
f(x)=\left\{\begin{array}{ccc}
x+\lambda & \text { for } & x>0 \\
0 & \text { for } & x=0 \\
x-\lambda & \text { for } & x<0
\end{array}\right.
$$


where $\lambda>0$, then it can be shown that his optimal strategy is as follows:

(a) Refuse to play with a positive fortune.

(b) Play with a nonpositive fortune.

(c) Stop if his fortune rises to one.

Thus the winning attitude produces behavior somewhat like that of many persons. 


\section{CHAPTER $\mathrm{X}$}

\section{GAMES IN EXTENSIVE FORM}

Because of the complexity of detail of the subject we shall confine ourselves to the stating of definitions and theorems of extensive game theory. In particular we shall rely heavily on examples to clarify concepts. Our basic reference will be Reference 12 .

A tree is a finite, partially ordered set that does not contain cycles. A rooted tree is a tree with a distinguished vertex. A game tree $\mathrm{K}$ is a finite, rooted tree with root 0 imbedded in an ordered plane. The vertex $\overline{0}$ corresponds to the start of the game, and the orientation of the plane is used to number the edges of the tree at a vertex. From any vertex $X$ of $K$ there is a unique path to 0 . The edges of the tree (if any) incident to $X$ and not lying on the path from 0 to $X$ are the alternatives at. $X$. Vertices that have alternatives are called moves and those that. do not (the "end points" of K) are called plays.

The alternative partition means the partition of the moves of $K$ into sets $A_{j}, j=1$, $2, \ldots$ where $A_{j}$ contains all of the moves with $j$ alternatives. The player partition means the partition of the moves of $K$ into $n+1$ sets $P_{0}, P_{i}, \ldots, P_{n}$. The moves in $P_{0}$ are called chance moves, and the moves in $P_{i}$ are called the personal moves of player $i$, for $i=1, \ldots, n$.

The information partition means a partition of $K$ into information sets $U$, which is a refinement of both the alternative and the player partitions, and such that no information set contains two moves on the same path from 0 to a play. Thus each $U$ is contained in $P_{i} \cap A_{j}$ for some $i$ and $j$. Let $U_{i}$ be the set of information sets for the ith player, and let $\mathcal{U}$ be the set of all information sets.

The pair $(\mathrm{K}, \mathcal{U})$ will be called a game structure.

On the information sets in $U_{0}$, the chance player, a probability distribution $p(U)$ is defined. Thus, if $U \in \boldsymbol{U}_{0}$ and $U \subset P_{0} \cap A_{j}$, then there is a positive function $p_{i}$ (U), $i=1, \ldots, j$, defined on the alternatives at $U$, such that $\sum p_{1}(U)=1$. These are the chance probabilities. (In ordinary games these probabilities are realized by shuffling cards, rolling dice, etc.)

Finally, on the play $W$ of $K$ there is defined a vector valued payoff function $h(W)=\left\langle h,(W), \ldots, h_{n}(W)\right\rangle$. The game starts at $U$ and end's at some play $W$, whereupon the players receive the amounts $h_{i}(W), \ldots, h_{n}(W)$.)

Given a choice of $K, U, h$, and $p$, we define an $n$-person game to be the collection $(K, \mathcal{U}, h, p)$. Most ordinary parlor games, providing that they have a rule that prevents infinite play (i.e., chess but not tennis), have rules that determine each of the above quantities, and hence determine an $\mathrm{n}$-person game. 
The way the game proceeds is now obvious. It starts at 0 and proceeds from move to move through the tree. The successor of a move is selected by the player in whose information set the move lies (by a chance device in the case of the chance player). After passing through a finite number of steps, a play is reached and the players receive payments as indicated by the payoff vector defined for that play.

DEFINITION. - - A pure strategy $\pi_{i}$ for player $i$ is a function from the sets $U$ in $U_{i}$ to the positive integers such that if $U \subset A_{j}$, then $\pi_{i}(U) \leq j$. The value of $\pi_{i}(U)$ is the index of the alternative chosen by the player at that information set.

Let $\Pi_{i}$ be the set of pure strategies for i. Let $\pi=\left(\pi_{1}, \ldots, \pi_{n}\right)$ denote an $n$-tuple of pure strategies, one for each personal player, and let $\Pi$ be the set of all $\pi$.

Given an $n$-tuple $\pi$, the chance probability distributions on chance moves induce a probability distribution $\mathrm{p}_{\pi}(\mathrm{W})$ on the plays $\mathrm{W}$ of. $\mathrm{K}$. Thus, if the pure strategies $\pi$ choose all the alternatives of personal moves on the path from $U$ to $W$, then $p_{\pi}(W)$ is the product of the chance probabilities on that path; otherwise $p_{\pi}(W)$ is zero. 'I'hen the expected payoff to player $i$ if strategy $\pi$ is used can be computed as $\mathrm{H}_{\mathrm{i}}(\pi)=\sum \mathrm{p}_{\pi}(\mathrm{W}) \mathrm{h}_{\mathrm{i}}(\mathrm{W})$. Also we can denote by $\mathrm{H}(\pi)=\left\langle\mathrm{H}_{1}(\pi), \ldots, \mathrm{H}_{\mathrm{n}}(\pi)\right\rangle$ the $\mathrm{n}$-tuple of payoffs if $\pi$ is used. Note that $H(\pi)$ depends on each $\pi_{i}$ and when expressed in matrix notation is an $\mathrm{n}$-dimensional array with vector entries. In the two-person, zero-sum case the array reduces to an ordinary matrix game. Let us call the array $H(\pi)$ the normal form of the game. The above discussion establishes the following result:

THEOREM 1. -- Every n-person game can be normalized, that is, put in normal form.

It is intuitively obvious that some are easy to solve; for example: board games (chess, checkers), tick-tack-toe, and other games in which each player can see the other player's "hand" and his moves. 'lhe players in these games possèss përtect informátín.

DEFINITION. - - An $n$-person game is said to have perfect information if all its personal information sets $U$ are unit sets, that is, contain a single move.

To indicate what is meant by a solution of an $n$-person game, equilibrium points will be discussed.

DEFINITION. - - Let $\pi / \rho_{i}$ denote the vector $\left\langle\pi_{1}, \ldots, \pi_{i}-1, \rho_{i}, \pi_{i+1}, \ldots, \pi_{n}\right\rangle$. Then $\pi$ is a pure strategy equilibrium if and only if

$$
h_{i}\left(\pi / \rho_{i}\right) \leq h_{i}(\pi)
$$

for all pure strategies $\rho_{i}$ and $i=1, \ldots, n$.

Intuitively, an equilibrium point is one at which no player can improve his own position by shifting to another of his pure strategies if the other players keep their strategies fixed.

THEOREM 2. - - Every game with perfect information possesses a pure strategy equilibrium point.

This theorem can be proved by starting at the end of the game and "working backwards" by an induction argument.

Not all games have perfect information; hence, the above concepts must be broadened. 
DEFINITION. - - A mixed strategy $\mu_{i}$ for player $i$ is a probability distribution on the pure strategies in $\Pi_{i}$. Let $\mu=\left\langle\mu_{1}, \ldots, \mu_{n}\right\rangle$ be an n-tuple of mixed strategies. Let $\mathrm{p}_{\pi}(\mu)$ be the product of the weights given to the $\pi_{i}$ in $\pi=\left\langle\pi_{1}, \ldots, \pi_{n}\right\rangle$. Then the expectation of the ith player is

$$
h_{i}(\mu)=\sum_{\pi} h_{i}(\pi) p_{\pi}(\mu),
$$

if mixed strategy $\mu$ is used by the players.

DEFINITION. - - Let $\mu / v_{i}$ be the $n$-tuple $\left\langle\mu_{1}, \ldots, \mu_{i-1}, v_{i}, \mu_{i}+1, \ldots, \mu_{n}\right\rangle$. Then $\mu$ is a mixed strategy equilibrium point if and only if

$$
h_{i}\left(\mu / v_{i}\right) \leq h_{i}(\mu)
$$

for all mixed strategies $\mu_{i}$ and $i=1, \ldots, n$.

THEOREM 3. -- Every n-person game has a mixed strategy equilibrium point.

This can be proved by an extension of the fixed-point proof of the minmax theorem given in Chapter I, Part B1.

For a two-person, zero-sum game, Theorem 3 reduces to the minmax theorem. There are objections to equilibrium-point solutions to $n$-person games because the solutions are not necessarily unique. Although many other solution concepts have been proposed for $\mathrm{n}$-person games, the equilibrium point theorem is the only general theorem that has been established. Hence, a solution from now on shall mean an equilibrium-point solution.

One of the interesting problems in $\mathrm{n}$-person game theory is the way in which information is distributed among the players. We shall study this problem, making use of the diagrams of games that appear in Figs. 7 and 8 . A number of definitions are necessary.

DEFINITION. - - Let $U_{v}$ be the set of moves in $K$ that follow moves in $U$ by the $v$ th alternative. Then $U$ is a signaling information set for player $i$ if, for some. $v$ and some information set $V$ of player $i$, we have $U_{v} \cap V \neq \phi$ and $V \nsubseteq U_{v}$.

DEFINITION. - - A game is said to have perfect recall if none of the players possesses signaling information sets.

Any game with perfect information obviously has perfect recall. Poker, Kriegspiel, gin rummy, etc., have perfect recall (but not perfect information). If the partners in bridge are regarded as a single player, then bridge is a two-person game that does not have perfect recall. The reader may check that the various games illustrated in Figs. 7 and 8 either do ur do nüt have perfect recall as indicated there. 


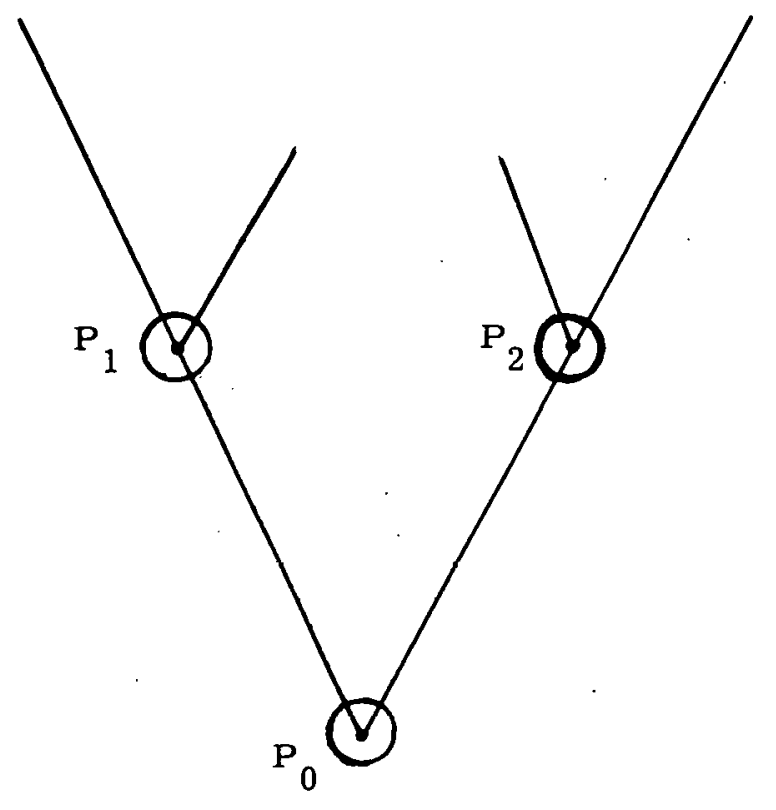

Both players have perfect information and perfect recall.

(a)

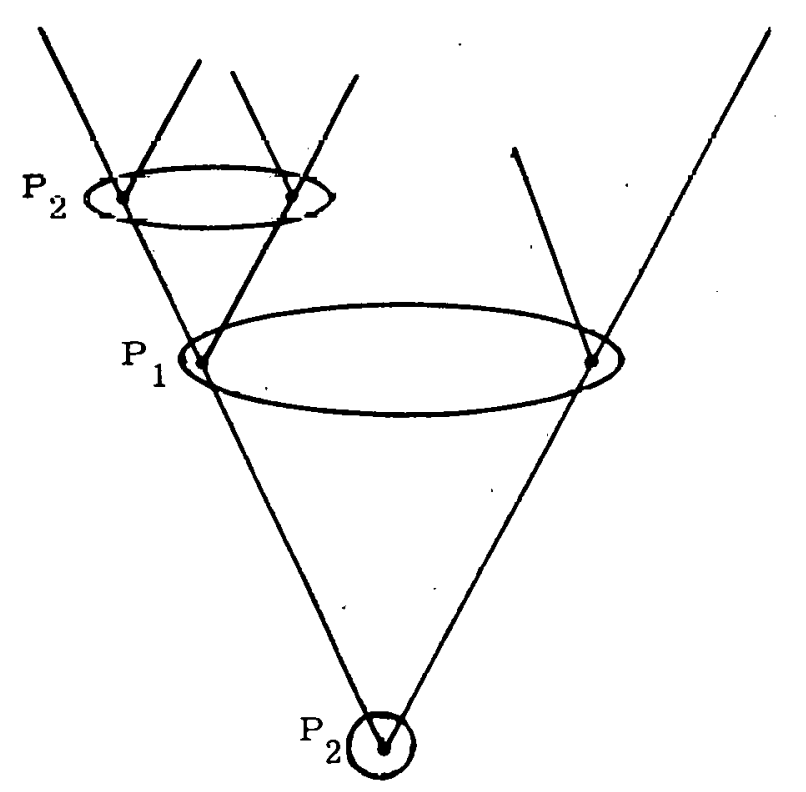

Neither player has effectively perfect information. Both have perfect recall.

(c)

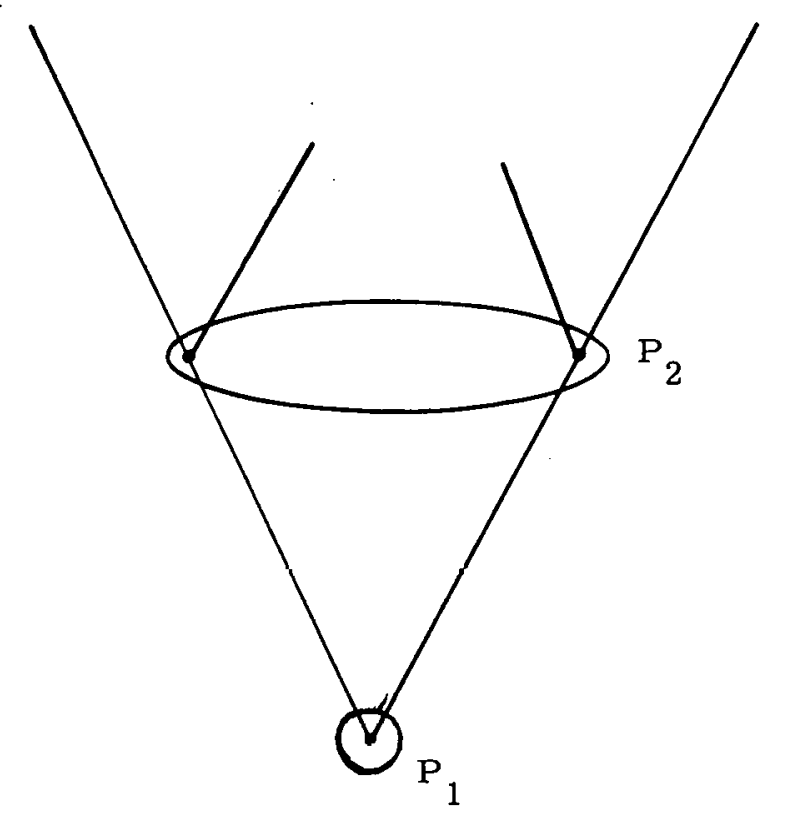

Both players have effectively perfect information and perfect recall.

(b)

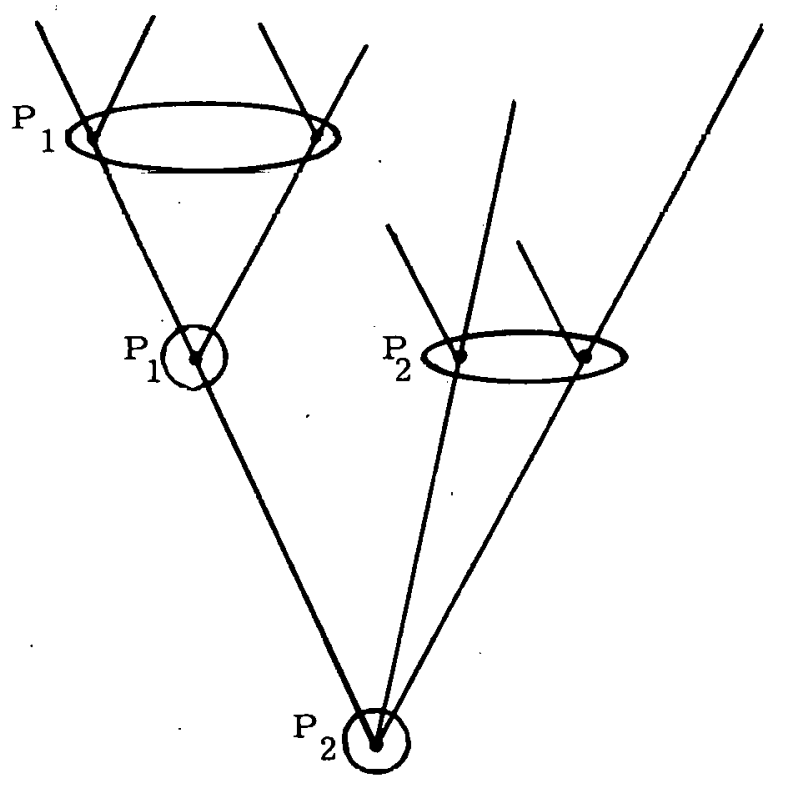

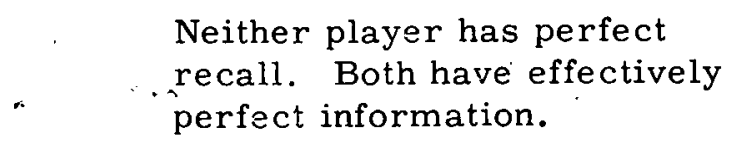

(d) 


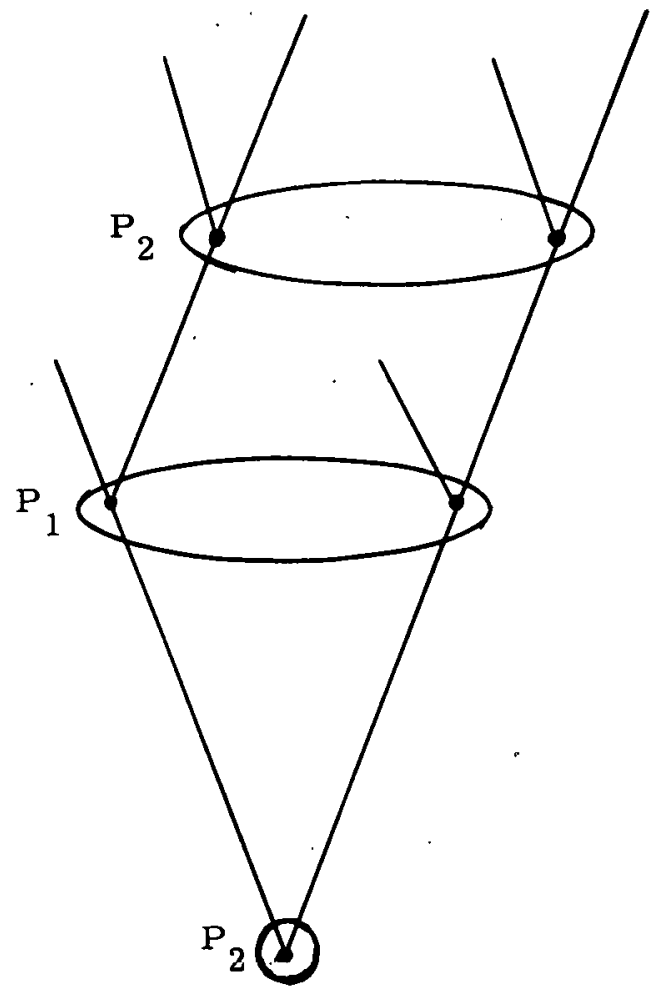

$\mathrm{P}_{2}$ has effectively perfect information but $P_{1}$ does not. $P_{1}$ has perfect recall but $P_{2}$ does not.

(a)

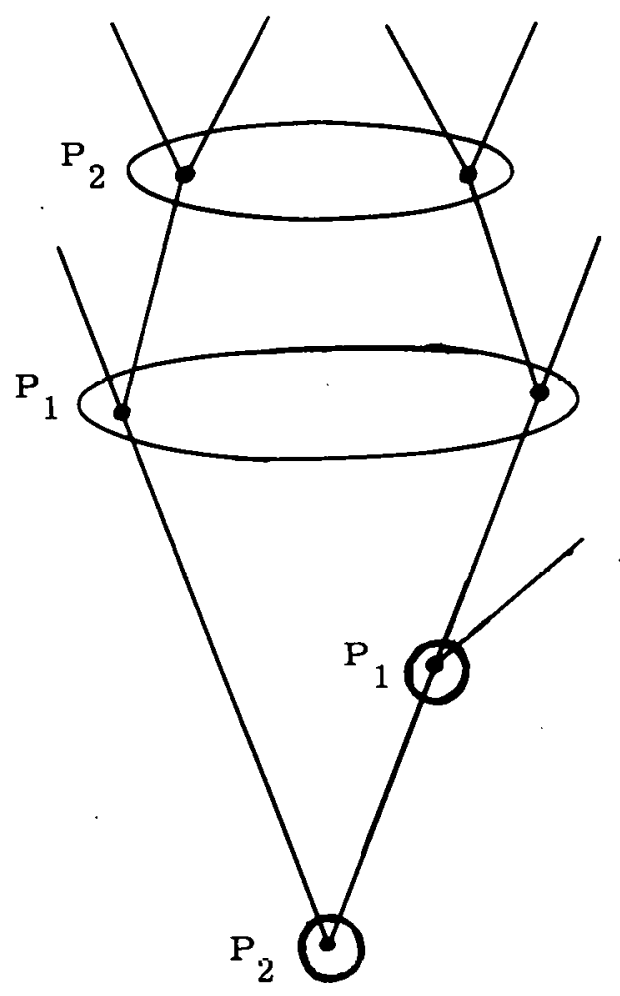

Neither player has either perfect recall or effectively perfect information.

(b)

Figure 8 
DEFINITION. - - A behavior strategy for player $i$ is an assignment, to each information set $U$ in $U_{i}$, of a probability distribution on the alternatives at $U$.

To every mixed strategy for a player, there corresponds a behavior strategy. This is a many-one correspondence since the dimension of the set of mixed strategies is larger than the dimension of the set of behavior strategies. Thus, if a game can be solved in behavior strategies, it is easier than solving it in mixed strategies.

THEOREM 4. - - A necessary and sufficient condition that the n-person game $(\mathrm{K}, U, \mathrm{~h}, \mathrm{p})$ has the same mixed strategy and behavior strategy equilibrium points for all $h$ and $p$ is that every player have perfect recall.

Thus poker, Kriegspiel, etc., may be solved using behavior strategies.

DEFINITION. - - A pure signaling strategy for player $i$ is a function $\sigma_{i}$ from his signaling information sets $U$ to the positive integers such that $1 \mathrm{l} U \mathrm{CA}_{j}$, then $o_{i}(U) \leqq J$. A mixed signaling strategy $v_{i}$ for player $i$ is a probability distribution on the pure signaling strategies for i.

A pure signaling strategy $\sigma_{i}$ singles out (for player $i$ ) a subtree $K \sigma_{i}$ of $K$. Let $U_{\sigma_{i}}$ be the set $U$ of information sets for player $i$ relativized to $K \sigma_{i}$.

DEFINITION. - - An associated behavior strategy is an assignment, to each information set in $\bigcup_{\sigma_{i}}$, of a probability distribution on the alternatives at that information set.

DEFINITION. - - A composite strategy is a pair consisting of a mixed signaling strategy and a set of associated behavior strategies.

THEOREM 5. -- Every n-person game has the sảme m1xed strategy and composite strategy equilibrium points. Hence every two-person, zero-sum game can be solved using composite strategies.

In order to use a composite strategy, the agents of a player meet before the game and decide, by a chance device, which signaling strategy to use. Their choice is kept secret from the other players. Then the agents who control signaling information sets make their choices according to the signaling strategy chosen, and the other agents use the associated behavior strategy on the nonsignaling information sets.

In bridge, for example, players use the order in which they lead or discard cards to signal what the other cards in their hands are -- or at least to indicate what the other cards are. They must also be careful not to signal to their opponents any more information than necessary.

A final question is related to the concept of perfect information. It was remarked earlier that a game with perfect information could be solved using pure strategies. This is a sufficient but not necessary condition.

DEFINITION. - - A game is said to have effectively perfect information when, for

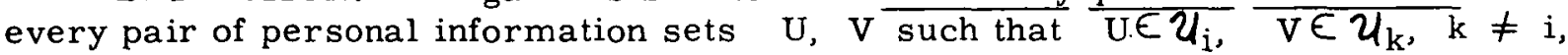
if $\mathrm{U}<\mathrm{V}$ then $\mathrm{V} \subset \mathrm{U}_{\mathrm{v}}$ for some $v$.

Intuitively, effectively perfect information exists if, whenever it is a player's turn, he remembers every previous move of his personal opponents and knows at least as much as they knew when they made these moves. 
DEFINITION. - - A game is essentially determinant if it has an equilibrium point in pure strategies for every assignment of $h$ and $p$.

THEOREM 6. - - A necessary and sufficient condition that a game be essentially determinant is that its complete inflation have effectively perfect information.

We shall not define the complete inflation of a game, but simply remark that it is an equivalent game which, in a sense, has the fewest information sets. All the games in Figs. 7 . and 8 are completely inflated.

It should now be possible for the reader to check, in Figs. 7 and 8 , that the above definitions are or are not satisfied as indicated. 
CHAPTER XI

MATHEMATICAL ECONOMICS AND MATRIX GAME THEORY

\section{A. Introduction}

This chapter will be concerned with two examples in which matrix game theory was used, purely as a mathematical tool, in an economic model. These models were formulated solely for use in economic theory, yet it turned out that game theory was the correct kind of mathematics needed to solve the problems arising in the model.

\section{B. A Generalization of the Von Neumann Model of an Expanding Economy}

(This section summarizes Reference 13, a paper by the same title by J. G. Kemeny, O. Morgenstern, and G. L. Thompson.)

1. We consider as a model of an economy a finite set of $m$ processes which operate at discrete time intervals and which produce a finite number $\mathrm{n}$ of different goods. From an economic point of view one usually has $m>n$ because, for most goods, there are alternate ways of production so that there is a choice among production processes. From a mathematical point of view we need not, and do not, assume any relationship between $m$ and $n$. The processes may be manufacturing processes, but they may also represent consumption, storage, and (as we show later) outside demand. We assume constant returns to scale and the unlimited availability of the natural factors of production such as labor and land. The inputs needed for the processes at any time $t$ are the goods produced during the preceding time period $t-1$, plus the natural factors of production.

Each process operates at any intensity $x$, where $x$ is a real nonnegative number. Intensities are normalized so that the $i$ th process operates at intensity $x_{i}$ where $0 \leq x_{i} \leq 1$ and $\sum_{i=1}^{m} x_{i}=1$. Thus the intensity vector (a row vector) $x=\left(x_{1}, \ldots, x_{m}\right.$ ) shall be viewed as an m-dimensional probability vector. When the ith process is operating, it requires $a_{i j}$ units of good $j(j=1, \ldots, n)$ and produces $b_{i k}$ units of good $k(k=1, \ldots, n)$ per unit of good i. Since only the ratios of these numbers are significant, the units may be chosen arbitrarily. It is assumed that $a_{i j}$ and $b_{i j}$ are nonnegative real numbers for all $i$ and $j$. Symbolically, physical production change during one time period can be represented as

$$
\text { (time } t-1) \quad x A \rightarrow x B \quad \text { (time } t \text { ), }
$$

where $A=\left\|a_{i j}\right\|$ and $B=\left\|b_{i j}\right\|$. The components of the vector $x A$ give the amounts of inputs used up in production, and the components of the vector $x B$ give the amounts produced. 
Each good* is assigned a price $y$, where $y$ is a nonnegative real number. Prices are also normalized so that the $j$ th good is assigned price $y_{j}$, where

$0 \leq y_{j} \leq 1$ and $\sum_{j=1}^{n} y_{j}=1$. Thus the price vector (a column vector) $y=\left(y_{1}, \ldots, y_{n}\right)$ is an $\mathrm{n}$-dimensional probability vector. Symbolically value changes during one time period can be represented as

$$
(\text { time } t-1) \quad \text { Ay } \rightarrow \text { By } \quad \text { (time } t) .
$$

The components of the vector $A_{y}$ give the value of the input entering into the processes, and the components of the vector $B_{y}$ give the value of goods produced by the processes.

It is assumed that there is an interest rate $b$ (percent) from which the interest factor $\beta=1+b / 100$ is derived. Interest is paid by an outside source, and investment is always possible. It is also assumed that there is an expansion rate a (percent) from which the expansion factor $a=1+a / 100$ is derived. Because of the assumed unlimited supplies of "land and labor," i.e., the original means of production, expansion can continue indefinitely.

We are looking for vectors $x$ and $y$ and numbers $\alpha$ and $\beta$ which satisfy the following five conditions. The first one is a conservation condition which says that no more goods can be used during any time period than were produced during the preceding time period. In equation form this reads

CONDITION $1 \quad x B \geq a \times A$ or $x(B-a A) \geq 0$.

(Here and elsewhere we shall use the convention that, if $u$ and $v$ are vectors, then $\mathrm{u} \geq \mathrm{v}$ shall mean that the corresponding inequalities shall be true for the components of $u$ and v. Also, we do not distinguish between the number zero and the zero vector since the context will always be clear.) The second condition makes the economy a profitless one; i.e., one in which a process cannot yield a return greater than that yielded by the going interest rate; in equation form this reads

CONDITION $2 \quad \beta A y \geq B y$ or $(B-\beta A) y \leq 0$.

The third condition requires that a zero price be charged for goods that are overproduced; in other words,

CONDITION $3 \quad \mathbf{x}(\mathbf{B}-\mathbf{a} A) \mathbf{y}=0$.

The fourth condition is

CONDITION $4 . \quad x(B-\beta A) y=0$,

which says that inefficient processes must be used with zero intensity.

* Labor, land, and other natural factors must be treated as free, since they are not produced. But there are simple means for entering the costs connected with these factors in the model; e.g., the cost of labor can be introduced in terms of the consumption of the worker and his family. In this approach consumer goods would be among the inputs of all processes. 
Without further assumptions about the matrices $A$ and $B$ there will, in general, be no solution satisfying these conditions. Von Neumann made the following additional assumption

$$
a_{i j}+b_{i j}>0, \quad \text { for all } i \text { and } j .
$$

Intuitively, this assumption means that every process must either consume or produce a positive amount of every good. Von Neumann made this assumption to insure the uniqueness of a and to prevent the economy from breaking up into disconnected parts. If we observe that the numbers $a_{i j}$ and $b_{i j}$ can be made very small, this assumption does not seem unreasonable; however, it has been criticized by economists. The condition is natural when the model is highly aggregated.

Observe that the following uninteresting economies satisfy all of the above conditions:

EXAMPLE 1. - Let $A$ and $B$ be matrices with $a_{i j}=1$ and $b_{i j}=0$ for all $i$ and $j$; let $a=\beta=0$, and let $x$ and $y$ be arbitrary probability vectors. It. is easy to see that these quantities satisfy Conditions 1-4 and (*). This is an economy which uses raw materials but produces nothing.

EXAMPLE 2. - Let $A$ and $B$ be matrices with $a_{i j}=0$ and $b_{i j}=1$ for all $i$ and $j$; let $a=\beta=\infty$, and let $x$ and $y$ be arbitrary probability vectors. It is easy to see that these quantities satisfy Conditions 1-4 and $(*)$. This is an economy which produces goods without using raw materials.

Neither of these examples corresponds to economic reality; they do, however, fit into the von Neumann model as special limiting cases. Here we want to weaken the (*) assumption; when we do so, we will find that examples like those mentioned above will become very annoying. We therefore impose an additional condition not contained in the original von Neumann model, namely

\section{CONDITION 5}

$$
\mathrm{xBy}>0 .
$$

Intuitively, this condition means that the total value of all goods produced must be positive. Observe that Example 1 does not satisfy this condition while Example 2 does.

Although we shall occasionally use Assumption (*) in this chapter, our principal assumptions will be the following pair of (to us, economically plausible) assumptions:

and

(i) every process uses some inputs, i.e., goods produced in the preceding time period,

(ii) every good can be produced in the economy; i.e., given a good, there exists at least one process which can produce 1 t.

These assumptions are much weaker than the von Neumann (*) assumption. They can be stated more precisely as follow:s:

(*) (i) every row of $A$ has at least one positive entry,

(ii) every column of $B$ has at least one positive entry.

Observe that neither of the examples above satisfies these conditions. 
The analysis that follows makes use of concepts and theorems from the theory of games of strategy. It will be assumed that the reader has a certain familiarity with game theory, especially with matrix games. We should like to point out that game theory is here used as a mathematical tool in order to obtain mathematical results (of which only those having economic meaning are admitted). Game theory appears therefore as a mathematical technique, comparable to, say, the calculus of variations or group theory. This use of the theory does not preclude its application to a large stationary or expanding economy in a very different sense, i.e., when the participants in the economy are viewed as playing a nonzero-sum, $\mathrm{n}$-person game. In the latter case, results may be obtained that are different from those shown in this chapter, especially because of the possibility of the formation of coalitions among the players. The emergence of the theory of games as a strictly mathematical tool for the analysis of more conventional economic situations, besides its role as a model of economic reality, is a noteworthy phenomenon and gives it added significance for the economist.

We now restate Assumptions (**) in game theoretical terms: Consider B and -A as matrix games, where the maximizing player controls the rows and the minimizing player controls the columns. Let $\mathrm{v}(\mathrm{B})$ and $\mathrm{v}(-\mathrm{A})$ be the values of each of these games. Then, remembering that the entries of $A$ and $B$ are nonnegative, it is easy to see that the (**) Assumptions are equivalent to the conditions

$$
\begin{aligned}
& v(-A)<0, \quad \text { and } \\
& v(B)>0 .
\end{aligned}
$$

If we have numbers $a$ and $\beta$ and vectors $x$ and $y$ which satisfy Conditions 1-5, then these quantities will provide solutions to the economic model which hold in every time period. We shall then say that the economy is in equilibrium.

2. We now interpret the whole problem in game-theoretic terms. It will become clear to the reader that some parts of the problem which are of game-theoretic interest are not of economic interest. We need the following lemma:

LEMMA 1. - - If $x, y, a$, and $\beta$ are solutions of Conditions 1-5, then

$$
a=\beta=x B y / x A y \text {. }
$$

PROOF. - - From Condition 5, we see that $x B y>0$; hence, from Conditions 3 and 4 , $x B y=a x A y=\beta x A y>0$. From the last equation $x A y>0$ so that $a=\beta=x B y / x A y$.

Thus we need look only for solutions in which $a=\beta$; i.e., the model requires that the interest rate should equal the expansion rate. Under this assumption Condition 4 becomes the same as Condition 3. Making the abbreviation $\mathrm{M}_{a}=\mathrm{B}-\mathrm{aA}$, Conditions 1, 2, and 5 be come

$\begin{array}{rlrl}\text { CONDITIONS } & 1^{\prime} & \mathrm{xM}_{a} \geq 0, \\ 2^{\prime} & \dot{\mathrm{M}}_{a} \mathrm{y} \leq 0, \text { and } \\ 5^{\prime} & \mathrm{xBy}>0 .\end{array}$


Observe that we have omitted Condition 3 (and its equivalent, Condition 4). This is permissible since, if we have a solution to Condition $1^{\prime}$ and $2 '$, then such solutions must satisfy Condition 3 as well. To see this, multiply Condition $1^{\prime}$ by $y$, obtaining $x M_{a} y \geq 0$; and multiply Condition $2^{\prime}$ by $x$, obtaining $x M_{a} y \leq 0$; these two expressions imply that $\mathrm{xM}_{\mathrm{a}} \mathrm{Y}=0$ which is Condition 3 .

If we interpret $M_{a}$ as a matrix game, where the maximizing player controls the rows and the minimizing player controls the columns, we see that Conditions $1^{\prime}$ and $2^{\prime}$ imply that $\left.\mathrm{vM}_{\mathrm{a}}\right)=0$. Moreover, Conditions $1^{\prime}$ and $2^{\prime}$ show that the solutions $\mathrm{x}$ and $\mathrm{y}$ to the economic problem are optimal strategies in the game $\mathrm{MI}_{a}$. We now restate our problem in gametheoretic terms.

PROBLEM. - - Given nonnegative $\mathrm{m} \times \mathrm{n}$ matrices $\mathrm{A}$ and $\mathrm{B}$ such that $\mathrm{v}(-\mathrm{A})<0$ and $v(B)>0$; set $M_{a}=B+a(-A)$, and find an $a$ so that $v\left(M_{a}\right)=0$; then find a pair of probability vectors $(x, y)$ such that $x B y>0$ and such that $x$ is optimal for the maximizing player, and $y$ is optimal for the minimizing player in the game $M$.

We shall call an a such that $v\left(\mathrm{M}_{a}\right)=0$ an allowable $a$. Even if we can find an allowable $a$, we will have to distinguish between two types of pairs of optimal strategies in the game $M_{a}$. If $(x, y)$ is a pair of optimal strategies for $M_{a}$, such that $x B y>0$, we shall call these economic solutions to the game $M_{a}$; on the other hand, if $\left(x^{\prime}, y^{\prime}\right)$ is a pair of optimal strategies for $M_{a}$, such that $x^{\prime} B^{\prime}=.0$, we shall call them noneconomic solutions to the game. $M_{a}$. It will turn out that if the expansion rate is not unique (and perhaps even if it is unique) then there always exist noneconomic solutions to the game. Since we are not interested in finding noneconomic solutions we shall not mention them again, and in this sense our problem becomes more economic than game-theoretic.

3. The purpose of this section is to discuss, under Assumptions (**), the existence of economic solutions to Conditions $1^{\prime}, 2^{\prime}$, and $5^{\prime}$.

Let $S_{m}$ be the set of all $m$-dimensivial pivbability vcctorc, and let $S_{11}$ he the. set of all $n$-dimensional probability vectors. In what follows, we shall usc $x \in S_{m}$ to denote a strategy for the maximizing player in $M_{a}$, and $y \in S_{n}$ to denote a strategy for the minimizing player in $\mathbf{M}_{\mathfrak{a}}$.

LEMMA 2. -- If $a^{\prime}$ and $a^{\prime \prime}\left(<a^{\prime}\right)$ are two distinct allowable values of a [i.e., $\left.v\left(M_{a^{\prime}}\right)=v\left(M_{a^{\prime \prime}}\right)=0\right]$, then $v\left(M_{a}\right)=0$ for $a$ in the interval $a^{\prime} \geq a \geq a^{\prime}$. Moreover, if $x^{\prime}$ is optimal in $M_{a^{\prime}}$ and $y^{\prime \prime}$ is optimal in $M_{a^{\prime \prime}}$, then the pair ( $\left.x^{\prime}, y^{\prime \prime}\right)$ is optimal in $M_{a}$ for all $a$ in the same interval.

PROOF.-- Let $x^{\prime}$ be an optimal strategy for the maximizing player in the game $M_{a^{\prime}}$; then $x^{\prime} M_{a} \geq 0$. If $a$ is any number less than $a^{\prime}$, we have

$$
x^{\prime} M_{a}=x^{\prime}(B-a A)=x^{\prime}\left(B-a^{\prime} A\right)+x^{\prime}\left(a^{\prime}-a\right) A \geq 0
$$

hence,

$$
v\left(\dot{M}_{\alpha}\right) \geq 0
$$


Similarly, let $y^{\prime \prime}$ be optimal for the minimizing player in $M_{a}$; then $M_{a} y^{\prime \prime} \leq 0$. If $a$ is any number greater than $a^{\prime \prime}$, we have

$$
M_{a} y^{\prime \prime}=(B-a A) y^{\prime \prime}=\left(B-a^{\prime \prime} A\right) y^{\prime \prime}+\left(a^{\prime \prime}-a\right) A y^{\prime \prime} \leq 0 ;
$$

hence,

$$
v\left(M_{a}\right) \leq 0 \text {. }
$$

The inequalities obtained at the conclusion of each of the two paragraphs above show that $v\left(M_{a}\right)=0$ and also show that. ( $\left.x^{\prime}, y^{\prime \prime}\right)$ are optimal strategies in the game $M_{a}$ for $a^{\prime} \geq a \geq a^{\prime \prime}$. This concludes the proof of the lemma.

COROLLARY. - - If (*) holds then there is at most one allowable a .

PROOF. - - Suppose there were two such, $a$ and $a^{\prime}$, with $a>a^{\prime}$. Let $(x, y)$ and $\left(x^{\prime}, y^{\prime}\right)$ be two economic solutions corresponding to these allowable $a^{\prime} s$. By the lemma, the pair $\left(x, y^{\prime}\right)$ is optimal at $a$ and $a^{\prime}$, so that

$$
x M_{a} y^{\prime}=x B y^{\prime}-a x A y^{\prime}=0
$$

and

$$
x M_{a_{1}} y^{\prime}=x B y^{\prime}-a^{\prime} x A y^{\prime}=0 .
$$

Subtracting these two equations, we have

$$
\left(a^{\prime}-a\right) \times A y^{\prime}=0 \text {; }
$$

subtracting a times the second from a' times the first, we get

$$
\left(a^{\prime}-a\right) \times B y^{\prime}=0
$$

hence, we see that $x A y^{\prime}=x B y^{\prime}=0$. Since $x$ and $y^{\prime}$ are probability vectors, we can choose indices $i$ and $j$ such that $x_{i} y_{j}>0$; then necessarily

$$
x_{i} a_{i j} y_{j}^{\prime}=x_{i} b_{i j} y_{j}^{\prime}=0
$$

so that $a_{i j}=b_{i j}=0$ which contradicts assumption $(*)$.

THEOREM 1. - - There are at most $\min (m, n)$ allowable a's for which economic solutions to $M_{\mathfrak{a}}$ exist.

PROOF. - For each such $a$, there is a pair $(x, y)$ so that $x B y>0$; hence, for each such $a$, we can choose components $\left(x_{i}, y_{j}\right)$ so that $x_{i} b_{i j} y_{j}>0$; hence, $x B^{j}>0$, and then Conditions 1 and 3 imply that $x A^{j}>0$. (A single superscript $j$ on a matrix indicates the jth column of that matrix.) We next show that the indices of the components so chosen are different for different such allowable $a^{\prime} s$. Let $\gamma$ and $\delta$ be two such allowable $a^{\prime} s$ with $Y>\delta$, and let the corresponding component pairs $\left(x_{i}, y_{j}\right)$ and $\left(x_{h}, y_{k}\right)$ be such that

$$
x_{i} b_{i j} y_{j}>0 \quad \text { and } \quad x_{h} b_{h k} y_{k}>0
$$


We must show that $i \neq h$ and $j \neq k$. We shall show $j \neq k$, and the proof of the other assertion is similar. Suppose, on the contrary, that $j=k$. By Lemma 2, there is a strategy $x$ (corresponding to $a=\gamma$ ) for the maximizing player which is optimal in $M_{a}$ for $\delta \leq a \leq \gamma$. Then, letting $M_{a}^{j}$ be the $j$ th column of $M_{a}$, we have

$$
x M^{j} Y \geq 0 \quad \text { and } \quad x M_{\delta}^{j} \geq 0
$$

However, since

$$
x M_{\delta}^{j}=\left(x B^{j}-Y x A^{j}\right)+(Y-8) x A^{j}=x M^{j} Y+(Y-8) x A^{j}
$$

and since

$$
(\gamma-8) \times A^{j}>0
$$

we see that

$$
\mathrm{xM}_{\delta}^{\mathrm{j}}=\mathrm{xM}_{\delta}^{\mathrm{k}}>0
$$

By Condition 3, this implies that $y_{k}=0$ which, in turn, implies $x_{h} b_{h k} y_{k}=0$, contrary to the way in which $y_{k}$ was chosen.

Since to each allowable a for which there are economic solutions there corresponds an entry $b_{i j}>0$ in the matrix $B$, and since the indices of two such entries are pairwise distinct, we see that the maximum number of such allowable $a^{\prime} s$ is equal to the longest diagonal which can be chosen in $B$. Because $B$ is an $m \times n$ matrix, the longest such diagonal is min $(m, n)$. This completes the proof of the theorem.

THEOREM 2 (Existence theorem).

(a) If (**) holds, then there is at least one and at most a finite number uf alluwable a's for which the game $M$ has economic solutions.

(b) (von Neumann) If (*) holds, then there is a unique allowable a.

(c). If (*) and (**) hold, then there is a unique allowable a moreover, for that $a$, the game $\mathrm{M}_{\mathfrak{a}}$ has economic solutions.

4. We shall now add to the von Neumann model the requirement that at each time period the economy should supply to an outside consumer a vector $d$ of goods already being produced by the economy. Hence, $d$ is an $1 \times \mathrm{n}$ row vector. We assume that $d$ is always a constant fraction of the output at any given time; i.e., the outsidc additional demand is expanding at the same rate as the economy.

The introduction of outside demand into the model opens up several new avenues of approach, some of which shall be studied or at least mentioned. The outside demand may be physically outside the economy, but it may also represent additional consumption by the workers within the economy. If viewed in the latter sense, we have removed the objection (noted in the introduction) to the original assumption that the model requires the restriction of the consumption by workers to the level of subsistence. As we shall see, the rates of expansion and the size of the outside demand can be closely connected. The result demonstrated below that decreasing the outside demand will allow a faster growth of the economy 
is economically very plausible and, indeed, corroborated from observations pertaining to economic development and the role of savings. The phenomenon has consequently been treated in business cycle analysis.

To simplify our equations, we assume that this external demand at time $t$. is supplied out of the production of time $t-1$. This assumption is consistent with the interpretation of the outside demand as added consumption; i.e., as the economy expands, the percentage of the total production going to the consumers remains constant. Our expressions now become

$\begin{array}{rlrl}\text { EXPRESSIONS } & 1 & x(B-a A) & \geq a d, \\ & 2 & (B-\beta A) y & \leq \beta e(d y), \\ 3 & x(B-a A) y & =a d y, \\ x(B-\beta A) y & =\beta d y, \text { and } \\ 4 & 5 & x B y & >0 .\end{array}$

In Expression 2 the vector $e$ is an $n \times 1$ (column) vector, each of whose entries is one. These expressions may be more briefly stated if we make greater use of the vector $e$. Observe that ed is an $m \times n$ matrix, each of whose rows is the vector $d$. Then we can write the above expressions as

$\begin{array}{ccc}\text { EXPRESSIONS } & 1^{\prime} & \mathrm{x}[\mathrm{B}-\mathrm{a}(\mathrm{A}+\mathrm{ed})] \geq 0, \\ 2^{\prime} & {[\mathrm{B}-\beta(\mathrm{A}+\mathrm{ed})] \mathrm{y} \leq 0,} \\ 3^{\prime} & \mathrm{x}[\mathrm{B}-\mathrm{a}(\mathrm{A}+\mathrm{ed})] \mathrm{y}=0, \\ 4^{\prime} & \mathrm{x}[\mathrm{B}-\beta(\mathrm{A}+\mathrm{ed})] \mathrm{y}=0, & \text { and } \\ 5^{\prime} & \mathrm{xBy}>0 .\end{array}$

Observe that these expressions correspond to Conditions 1-5 in Section B. 1 if we substitute the matrix $A^{\prime}=A+$ ed for $A$. Hence, all of the preceding work holds and the existence theorem insures that at least one economic solution exists.

THEOREM 3.-- The introduction of outside demand into the economic model has the following effect. Consider a subeconomy of the economy having a unique expansion factor.

(a) If the outside demand includes any good (produced by the subeconomy) which has a positive price, then the expansion factor of the (sub) economy must be decreased in order to supply the outside demand.

* The possibility of introducing cyclical components into the outside demand and hence into the behavior of the cntirc system easily suygests itself, but we chose not to proceed in

... that direction at present. 
(b) If the outside demand for goods (produced by the subeconomy) having a positive price is decreased, then the expansion factor can correspondingly be increased.

\section{The Walras-Wald Economic Model}

(This is a summary of unpublished work by G. L. Thompson and is closely related to work by H. W. Kuhn. See Reference 14.)

1. The equilibrium theorem of $A$. Wald was one of the first rigorous existence theorems in economics and alerted economists to the fact that the mere counting of the number of equations and variables was not an adequate method of establishing the existence of solutions to sets of equations. Wald's original papers are difficult to read and have been largely inaccessible. Therefore the recent elegant reformulation by $\mathrm{H}$. W. Kuhn of these results is to be welcomed. Kuhn was able to achieve a great simplification in the statements and proofs of theorems by using the recently developed theory of linear programming and the Kakutani fixed-point theorem. He also extended Wald's results by relaxing some of the conditions and assumptions that Wald required.

In reading Kuhn's paper, I was struck by a certain asymmetry in the formulation of the economic model and was led to try to generalize the model to make it more symmetric. This section reports on that generalization. I begin with Kuhn's formulation and obtain a new model by relaxing some of his conditions. It is to be emphasized, however, that the present paper parallels Kuhn's development in the main; the importance of the generalization given here is largely economic rather than mathematical.

The economic model is formulated in Section C. 2 and the economic meaning of the new conditions are discussed in Section C. 3. The assumptions necessary for and the proof of the existence theorem are given in Section C. 4 .

2. Consider the following model of din economy: There are $m$ factors of production and $n$ goods. The quantity of the ith factur ulilized by tho economy is indinated by $r_{i}$ (units arbitrary) and its price by $\rho_{i}$; similarly, llie quantity of the $j$ th gnnd produced by the economy is indicated by $s_{j}$ (units arbitrary) and its price by $\sigma_{j}$; the profit made on (or charged to) the $j$ th good is $\pi_{j}$; finally the quantity of the $j$ th good that is stockpiled by the economy is $a_{j}$. It is assumed that $a_{i j}$ units of the ith factor are needed to produce one unit of the $j$ th good, where $1=1, \ldots, 11$ and $j-1, \ldots, n$. The $m \times n$ matrix $A$ is the technological matrix of the economy.

To keep these various quantities straight we summarize them in.veclor form in Table III. Also included for future use are the constant vectors $e$ and $f$ which have each of their components equal to 1 .

In the economy, the quantities $s$ and $p$ are the independent variables. Once they are fixed, the other quantities are determined as functions of them. Thus, if $s$ is fixed, then the price of the $j$ th good is determined by the $j$ th demand function $\sigma_{j}$; that is $\sigma_{j}=\sigma_{j}(s)$; also, the profit made on the $j$ th good is determined by the $j$ th profit function $\pi_{j}$; that is, $\pi_{j}=\pi_{j}(s)$. Similarly, if $p$ is fixed, then the quantity of the ith factor used is determined by the ith factor endowment (or supply) function $r_{i}$; that is, $r_{i}=r_{i}(\rho)$; also, the quantity of the ith factor that is stockpiled is determined by the ith stockpiling function $q_{i}$; that is, $q_{i}=q_{i}(p)$. 


\begin{tabular}{cll} 
Vector & \multicolumn{1}{c}{ Kind } & \multicolumn{1}{c}{ Description } \\
$\rho$ & row m-vector & factor price vector \\
$r$ & column m-vector & factor endowment vector \\
$q$ & column m-vector & factor stockpiling vector \\
e & column m-vector & all components equal to 1 \\
$\mathrm{~s}$ & column n-vector & goods quantity vector \\
$\sigma$ & row n-vector & goods price vector \\
$\pi$ & row n-vector & goods profit vector \\
$\mathrm{f}$ & row n-vector & all components equal to 1
\end{tabular}

The functions $\sigma, \pi, r$, and $q$ and the matrix $A$ are assumed given and fixed for the economy. By an equilibrium in the economy we shall mean vectors $\bar{r}, \bar{s}, \bar{q}, \bar{\rho}, \bar{\sigma}$, and $\bar{\pi}$ that satisfy the six conditions given below. (In stating these conditions, we use the convention that if $u$ and $v$ are vectors with the same number of components, then $u \geq v$ shall mean that the inequality holds for corresponding components of these vectors.)

$\begin{array}{lll}\text { CONDITIONS } & \bar{r} \geq 0, \bar{s} \geq 0, \bar{\rho} \geq 0, \bar{\sigma} \geq 0 . \\ & \bar{\rho} A \geq \bar{\sigma}-\bar{\pi} . \\ & \\ 3 & A \bar{s} \leq \bar{r}-\bar{q} . \\ 4 & \bar{\rho}[A-e(\bar{\sigma}-\bar{\pi})] \bar{s}=0 . \\ 5 & \bar{\rho}[A-(\bar{r}-\bar{q}) f] \bar{s}=0 . \\ 6 & \bar{\sigma}=\sigma(\bar{s}), \bar{\pi}=\pi(\bar{s}), \bar{r}=r(\bar{p}), \bar{q}=q(\bar{\rho}) .\end{array}$

Let us interpret these equations economically. Condition 1 says that to have economic significance, prices and quantities must be nonnegative. Condition 2 requires that the cost of making a good be at least as great as its price less the profit made on it. Condition 3 states that the amounts of the factors used be at most the factor endowment less the amounts stockpiled. Condition 4 may be restated as follows: if $\sum_{i} \rho_{i}\left[a_{i j}-\left(\sigma_{j}-\pi_{j}\right)\right]>0$, then $s_{j}=0$; that is, if the cost of producing a good is greater than its price less its profit, then it is not produced. Similarly, Condition 5 may be restaled as $: \sum_{j}\left[a_{i j}-\left(r_{i}-q_{i}\right)\right] s_{j}<\dot{0}$, then $\rho_{i}=0$; that is, factors that are present in excess get zero price. Finally, Condition 6 states that the prices of goods and the profits made on goods are functions of the quantity of goods produced, and the factor endowments and amounts stockpiled are functions of the prices of the factors. 
REMARKS. - - For the moment, set $\pi$ and $q$ equal to zero, and set $r$ equal to a positive constant vector; then Conditions $1,3,4,5$, and 6 appeared in a similar way in Wald!s formulation, while Condition 2 appeared as an equality rather than an inequality. The inequality form of Condition 2 is due to Kuhn. The functions $\pi(s), r(\rho)$, and $q(\rho)$ are new in the present paper. From a mathematical point of view, the function $\pi$ could be absorbed in the function $a$, and the function $q$ could be absorbed in $r$. However, for economic interpretations it is desirable to keep them separate. Note that the model does not require that $\pi$ and $\mathrm{q}$ be nonnegative since negative profits (losses or subsidies) and negative stockpiles (imports) have obvious interpretations.

3. The most important of the new functions introduced here is the factor limitation (or supply) function $r(\rho)$. In the Wald model this function is constant, which for some economic interpretations is a reasonable restriction, but not for all. For example, if steel and concrete are considered as factors for building construction, then there is a fixed amount of earh available, and when either is used up construction must stop. Here a constant factor limitation function is applicable. On the other hand, if reinforced concrete and conoreteencased girders are regarded as factors of production, then, in first approximation, these materials can continuously be substituted for each other in buildings, and a linear type of limitation function is in order. Again, in cases where there is substitulability betwcen goods, as for example between wood and metal, or wood and plastic, for certain uses, then a nonconstant limitation function is suitable. In general, wherever there is substitutability or interchangeability among factors, a nonconstant factor limitation function arises.

The stockpiling function $q(p)$ is introduced to take case of certain factors whose production and.price are not determined by equilibrium conditions in the economy. For example, in the United States, gold, uranium, and certain farm products are stored by the government. Also, if the economy is receiving outside aid in the form of goods, then this ran be expressed by making some components of $\mathrm{q}$ negative.

The profit function $\pi(s)$ is introduced so that certain unprofitable goods that otherwise might not be produced may be subsidized by the economy. Such are, for example, certain farm products, research products, military weapons, etc.

There is a close relationship between the functions $q$ and $\pi$ and the quantities $p$ and $f$ on page 124 of Reference 15. They each permit a little slack in what otherwise would be very rigid price and production rules.

4. Given a technological matrix $A$ and fixed vectors $\bar{r}, \bar{\sigma}, \bar{q}$, and $\bar{\pi}$, we call define (following Kuhn) the following pair of dual linear programs:

(i) Maximum Problem. - Maximize the quantity $(\bar{\sigma}-\bar{\pi}) \mathrm{s}$, subject to the constraints $\bar{s} \geq 0$ and $A s \leq \overline{\bar{r}}-\bar{q}$.

(ii) Minimum Problem.-- Minimize the quantity $\rho(\bar{r}-\bar{q})$, subject to the constraints $\rho \geq 0$ and $\rho A \geq \bar{\sigma}-\bar{\pi}$.

These problems have the following economic interpretations: The quantity $(\bar{\sigma}-\bar{\pi})_{\mathrm{s}}$ is the net value of the goods produced by the economy, that is, their total value less the profit charged on them. The quantity $(\bar{r}-\bar{q})$ gives the net factor cost, that is, the cost of all factors less the cost of those stockpiled. These two problems taken together indicate that the economy is trying to maximize the net value of the goods it produces, while, at the same time, minimizing the net cost of the factors necessary to produce them. 
It is well-known that if either of these problems is solvable then both are, and there is a common value for the maximum value found in (i) and the minimum value found in (ii). Thus if $\bar{s}$ and $\bar{\rho}$ solve (i) and (ii), respectively, then

$$
(\bar{\sigma}-\bar{\pi}) \bar{s}=\bar{\rho}(\bar{r}-\bar{q}) .
$$

Conversely, if $\overline{\mathbf{s}}$ and $\bar{\rho}$ satisfy the constraints given in (i) and (ii) above, and if they also satisfy Assumption (*), then they solve the maximum and minimum problems stated above. In economic terms, Assumption (*) means that, at equilibrium, the net value of goods produced equals the net cost of factors used.

THEOREM 1 .

(a) If $\bar{r}, \bar{s}, \bar{q}, \bar{p}, \bar{o}$, and $\bar{\pi}$ satisfy Conditions $1-5$, then they solve the dual linear programs (i) and (ii), stated above.

(b) If $\bar{s}$, and $\bar{\rho}$, solve the dual linear programming associated with the vectors $\overline{\mathbf{r}} \geq 0, \bar{\sigma} \geq 0, \overline{\mathrm{q}}$, and $\bar{\pi}$, then Conditions $1-5$ are satisfied.

PROOF .

(a) From Conditions 1, 2, and 3, the vectors $\bar{s}$ and $\bar{\rho}$ satisfy the constraints of the linear programming problems. From Conditions 4 and 5 , we have

$$
\bar{\rho} A \bar{s}=\bar{\rho}(\bar{r}-\bar{q})=(\bar{\sigma}-\bar{\pi}) \bar{s},
$$

which is $(*)$, so that, by the theorem referred to above, the vectors $\bar{s}$ and $\overline{\dot{\rho}}$ solve the dual programs (i) and (ii).

(b) Conditions 1, 2, and 3 hold from the assumptions stated in (b). and the fact that the constraints of the linedr programs are satisfied. 'T'o show that Conditions 4 and 5 hold, we observe that

$$
\bar{\rho} A \bar{s} \geq(\bar{\sigma}-\bar{\pi}) \bar{s}=\bar{\rho}(\bar{r}-\bar{q}) \geq \bar{\rho} \Lambda \bar{s},
$$

where we have used successively Expressions 2, (*), and 3. Since the first and last terms of this chain of inequalities are equal, equality holds throughout. If two of the equalities thus obtained are rewritten, it is easy to show that Conditions 4 and 5 hold.

An equilibrium need not exist unless additional assumptions are made. In his original paper, Wald made assumptions sufficiently strong to get a unique equilibrium. Here we make a number of assumptions but only enough to give the existence, not the uniqueness, of an equilibrium. Kuhn's modifications of the Wald model also has the latter properly.

Our assumptions may be stated as follows:

$i$ and $\mathrm{j}$.

(A) The entries of the technological matrix are nonnegative; i.e., $a_{i j} \geq 0$ for all 
(B) For every $i$ there is a $j$ such that $a_{i j}>0$, and for every $j$ there is an $i$ such that $a_{i j}>0$; that is, every factor produces at least one good and every good is produced by at least one factor.

(C) The functions $r(p), q(p), o(s)$, and $\pi(s)$ are defined, bounded and continuous, and $r$ and $\sigma$ are nonnegative, for all nonnegative $\rho$ and $s$.

(D) The inequalities $r(\rho)-q(\rho) \geq 0$ and $\sigma(s)-\pi(s) \geq 0$ are satisfied for all nonnegative $\rho$ and $s$. In other words, it is impossible to stockpile larger quantities of factors than exist, and no profit on a good can be charged in excess of the price of that good.

(E) There are $n$ functions $t_{j}(s)$ such that, for each $s>0$, there is a $\rho$ satisfying the inequalities

$$
o(s)-\pi(s) \leq \rho A \leq t(s) .
$$

Moreover, the functions $t$ are defined and bounded for all nonegalive $s$. 'l'his requirement prevents unbounded inflationary pressures on factor prices and insures the possibility of determining factor prices in the economy.

THEOREM 2. -- If A, r, q, $\sigma$, and $\pi$ satisfy Assumptions (A)-(E), then an economic equilibrium exists. 


\section{REFERENCES FOR PART THREE}

1. Kemeny, J. G., Snell, J. L., and Thompson, G. L.

Introduction to Finite Mathematics,

Prentice-Hall, New York, 1957.

2. Nash, John

Non-Cooperative Games,

Annals of Mathematics. (2)'54, pp 286-295, 1951.

3. Gale, D., Kuhn, H. W., and Tucker, A. W.

On Symmetric Games,

Contributions to the Theory of Games, Vol I, Annals of Mathematics Studies, Number 24, Princeton University Press, Princeton, N. J., 1950.

4. Stiemke, E.

Uber positive Lösungen homogener linearer Gleichungen,

Mathematische Annalen, 76, 1915, pp 340-342.

5. Tucker, A. W.

Dual Systems of Homogeneous Linear Relations,

Linear Inequalities and Related Systems, Annals of Mathematics Studies,

Number 38, Princeton University Press, Princeton, N. J., 1956.

6. Gale, D., and Sherman, S.

Solutions of Finite Two-Person Games,

Contributions to the Theory of Games, Vol I, Annals of Mathematics Studies, Number 24, Frinceton Universily Press, Princeton, N. J., 1950.

7. Bohnenblust, H. F., Karlin, S., and Shapley, L. S.

Solutions of Discrete, Two-Person Games,

Cuntribuliuns to the Theory of Games, Vol I, Annals of Mathematics Studies, Number 24, Princeton University Press, Princeton, N. J., 1950.

8. Shapley, L. S., and Snow, R. N.

Basic Solutions of Discrete Games,

Contributions to the Theory of Games, Vol I, Annals of Mathematics Studies, Number 24, Princeton University Press, Princeton, N. J., 1950.

9. Cowles Commission for Research in Economics.

Activity Analysis of Production and Allocation, edited by T. C. Koopmans, Wiley, New York, 1951. 


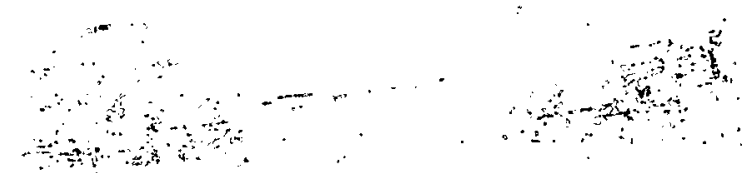

10. Robinson, J.

An Iterative Method of Solving a Game,

Annals of Mathematics, (2) 54, 1951.

11. Mosteller, Frederick and Nagel, Philip

An Experimental Measure of Utility,

The Journal of Political Economy, Vol LIX, Number 5, October 1951, pp 371-404.

12. Games in Extensive Form

Contributions to the Theory of Games, Vol II, Annals of Mathematics Studies, Number 28, edited by H. W. Kuhn, and A. W. Tucker, Princeton University

Press, Princeton, N. J., 1953.

13. Kemeny, J. G., Morgenstern, O., and Thompson, G. L.

A Generalization of the Von Neumann Model of an Expanding Economy,

Econometrica, 24, 1956, pp 115-135.

14. Kuhn, H. W.

On a Theorem of Waid,

Linear Inequalities and Related Systems, Annals of Mathematics Studies,

Number 38, Princeton University Press, Princeton, N. J., 1956.

15. Kemeny, J. G., Morgenstern, O., and Thompson, G. L.

A Generalization of the Von Neumann Model of an Expanding Economy,

Econometrica, Vol 24, Number 2, April 1956. 
Issued by

Technical Information Division

Sandia Corporation

Albuquerque, New Mexico 